\title{
The effectiveness of total surface bearing compared to specific surface bearing prosthetic socket design on health outcomes of adults with a trans-tibial amputation: a systematic review
}

\author{
A thesis submitted by \\ Sally Jane Cavenett \\ in partial fulfilment of the requirements for the Degree of \\ Master of Clinical Science (MClinSc)
}

The Joanna Briggs Institute

School of Translational Health Science

Faculty of Health Sciences

University of Adelaide

February $14^{\text {th }} 2014$ 


\section{CONTENTS}

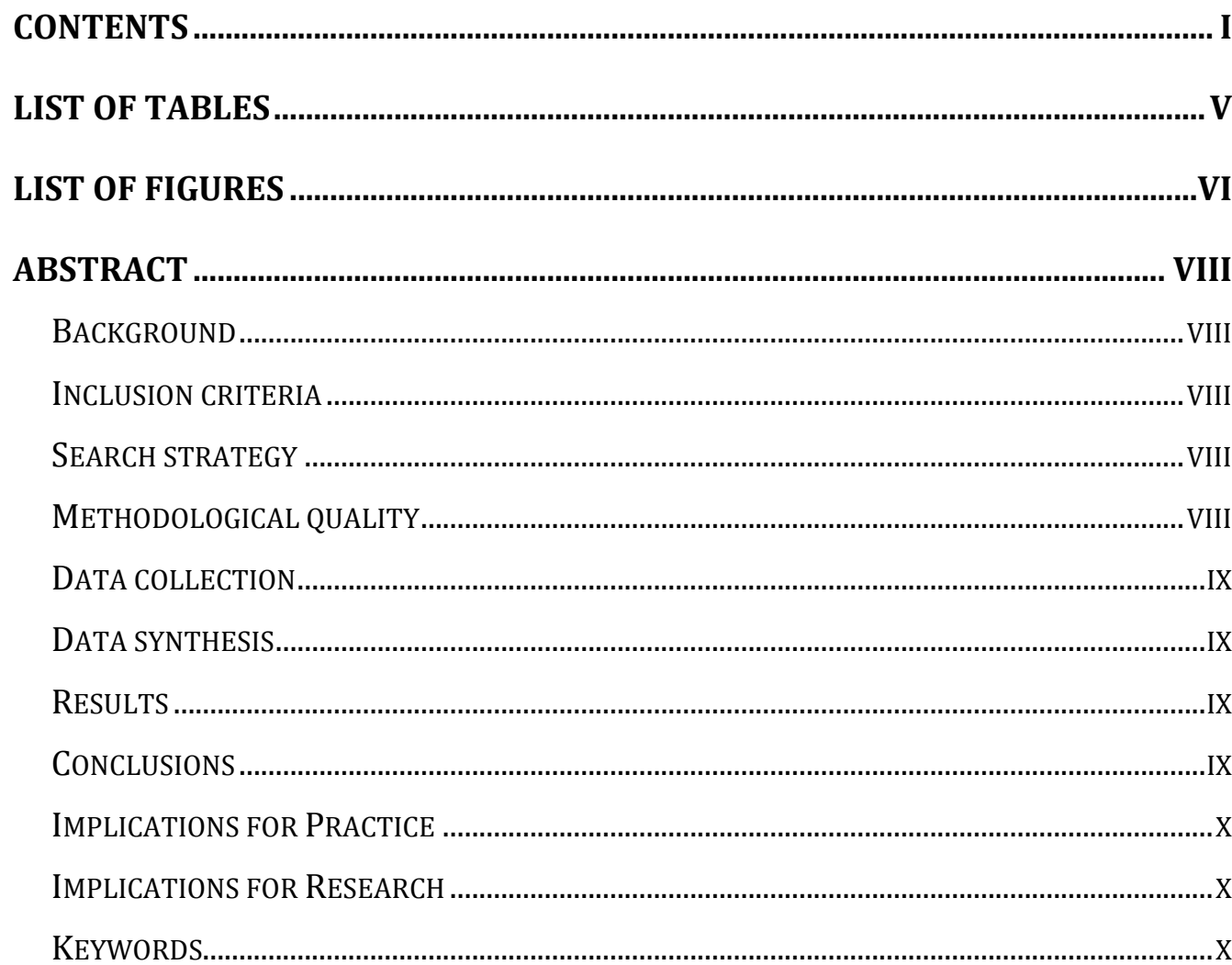

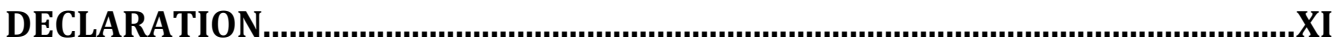

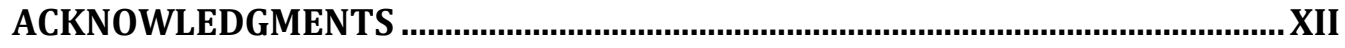

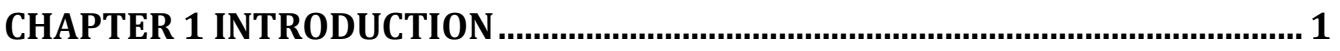

THIS FIRST CHAPTER OF THE THESIS DESCRIBES THE CONTEXT OF THE REVIEW (SECTION 1.1); WITH REFERENCE TO THE EXISTING RESEARCH ON PROSTHETICS AND DESCRIPTION OF SOCKET DESIGNS, AND THE MOTIVATION FOR THE RESEARCH INTRODUCED (SECTION 1.2); THE PURPOSE OF THE SYSTEMATIC REVIEW IS DEFINED (SECTION 1.3) AND AN OUTLINE OF THE STRUCTURE OF THE THESIS IS PROVIDED (SECTION 1.4) ........................... 1

1.1. CONTEXT FOR THE REVIEW .................................................................................... 1

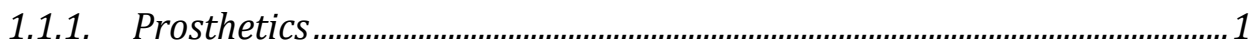

1.1.2. Prosthetic prescription .................................................................................. 2

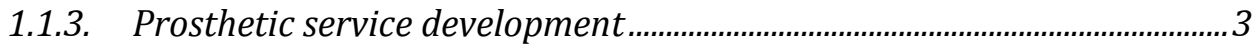

1.1.4. Factors that influence socket prescription and outcomes: Cause and level of amputation, age, and componentry.......................................................... 4

1.1.5. Goals of prosthetic rehabilitation................................................................ 6 
1.1.6. The TSB and SSB prosthetic socket designs: Description and rationale for their selection.

1.1.6.1. The SSB socket design $\quad$....................................................................

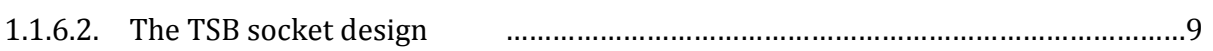

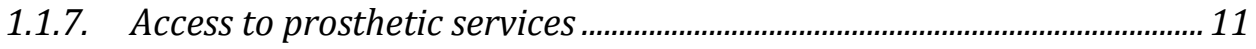

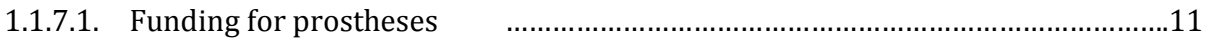

1.1.8. Conceptualisation of outcomes ...................................................................... 12

1.2. MotiVATION FOR THE RESEARCH: THE VALUE OF THE SYSTEMATIC REVIEW...............12

1.3. SYSTEMATIC REVIEW AND EVIDENCE BASED HEALTHCARE ........................................15

1.4. OVERARCHING RESEARCH OBJECTIVE ..................................................................17

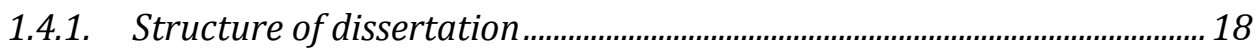

CHAPTER 2 SYSTEMATIC REVIEW METHODS .................................................19

2.1. TYPES OF STUDIES.............................................................................................19

2.2. TYPES OF PARTICIPANTS..................................................................................19

2.3. TYPES OF INTERVENTIONS AND COMPARATORS ……...............................................19

2.4. TYPES OF OUTCOMES AND OUTCOME MEASURES.....................................................20

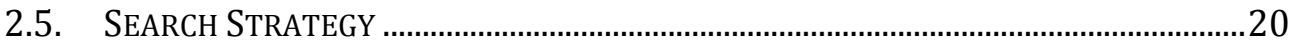

2.6 ASSESSMENT OF METHODOLOGICAL QUALITY AND APPROACH TO STUDY INCLUSION

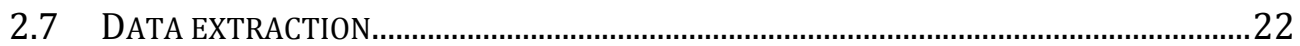

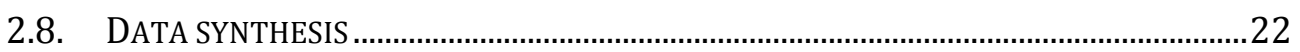

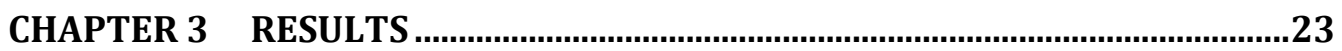

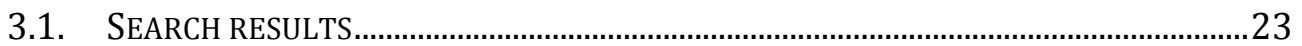

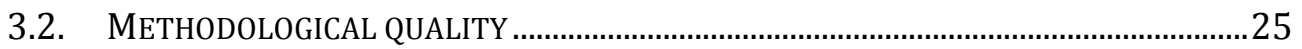

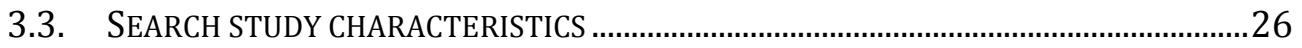

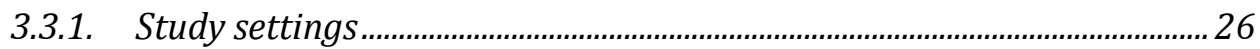

3.3.2. Interventions and comparators................................................................... 27

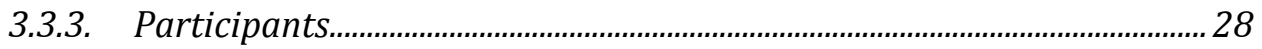

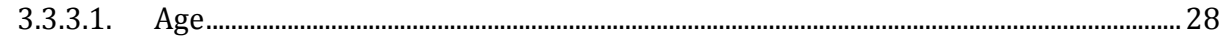

3.3.3.2. Co-morbidities, baseline activity, and years since amputation .................................. 28

3.3.3.3. Aetiology / cause of amputation...............................................................................................28

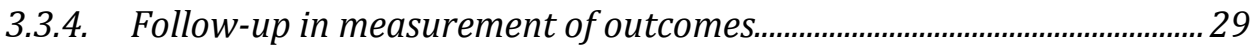

3.4. FINDINGS OF THE REVIEW ……………………..................................................29

3.4.1. Reported outcomes and measurement scales/instruments....................29

3.4.2. Characteristics of individual studies ….................................................... 31 


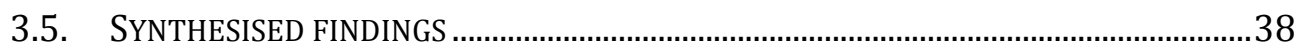

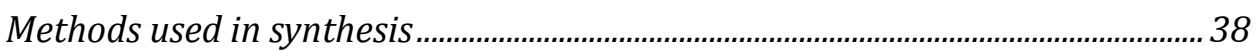

Synthesised results by health outcome domain .........................................................38

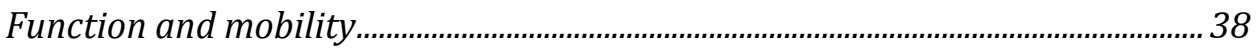

3.5.1. Laboratory-based physical performance measures.................................... 39

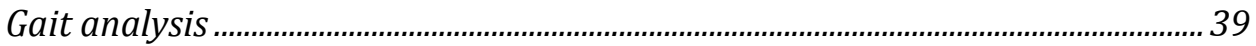

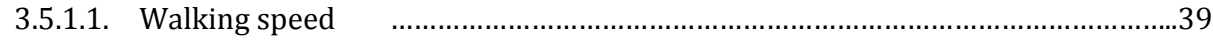

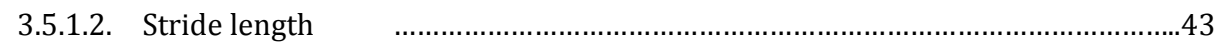

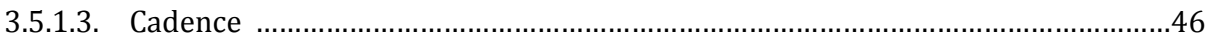

Asymmetries in gait: spatial and temporal............................................................. 48

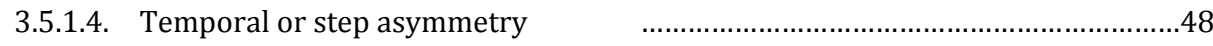

3.5.1.5. Spatial or stance asymmetry $\quad$...............................................................

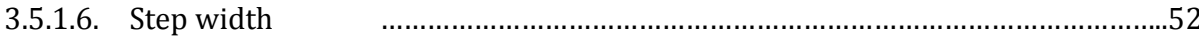

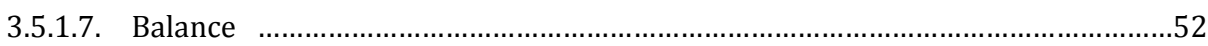

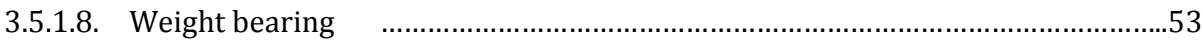

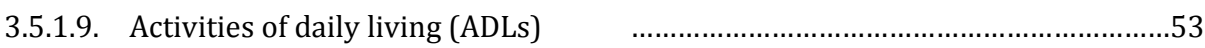

3.5.2. Function and mobility - Community based physical performance

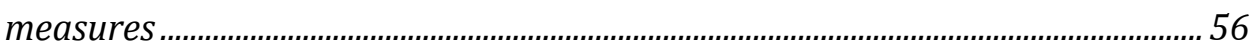

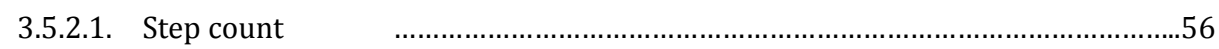

3.5.2.2. Prosthetic wear time $\quad$..............................................................................5

3.5.3. Function and mobility: Questionnaire .....................................................5

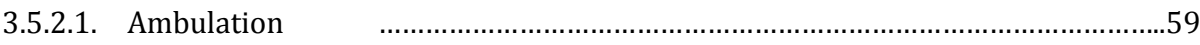

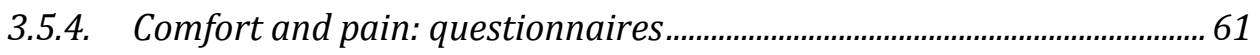

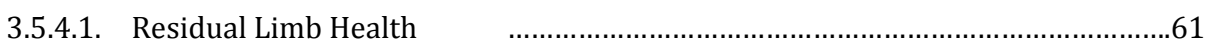

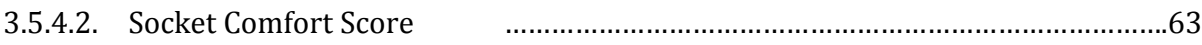

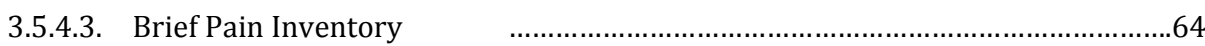

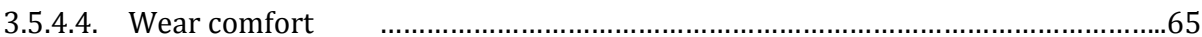

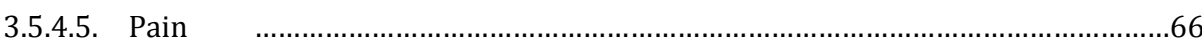

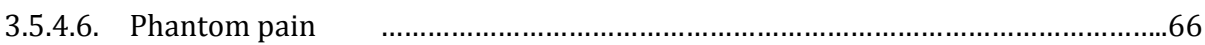

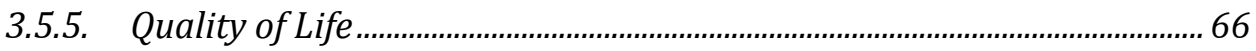

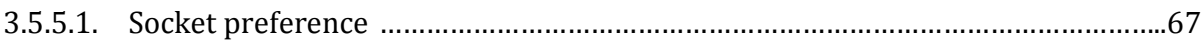

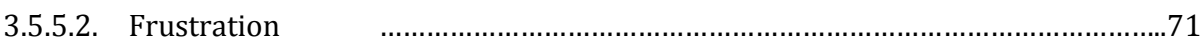

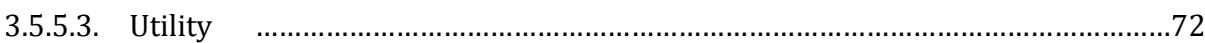

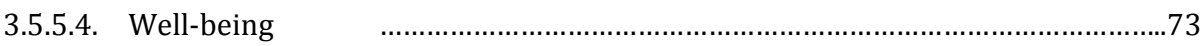

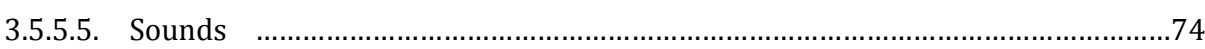

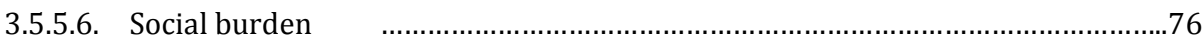

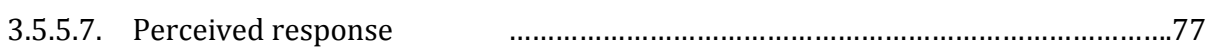

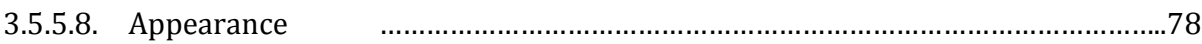

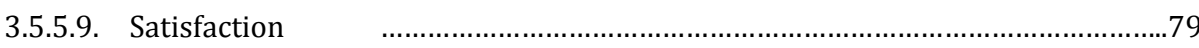

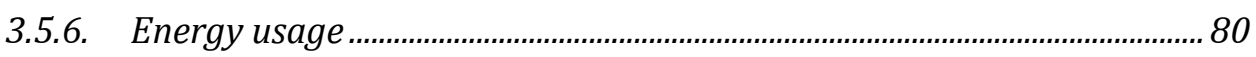




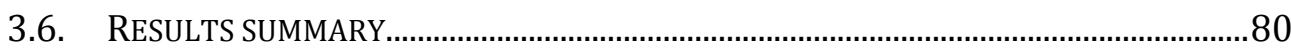

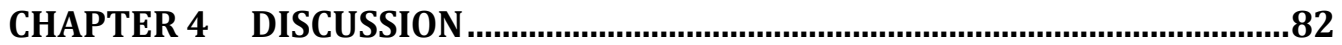

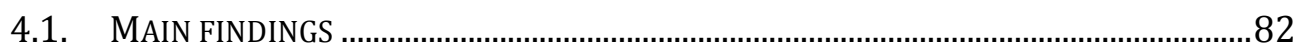

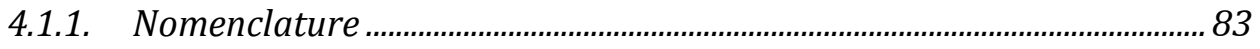

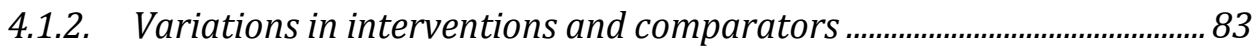

4.1.3. Variation in study objectives/intention …................................................ 84

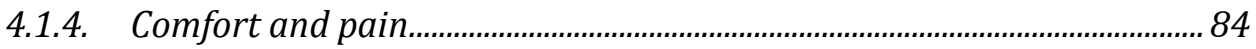

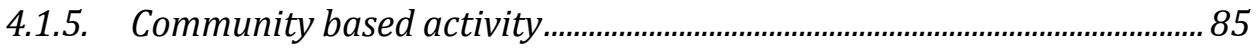

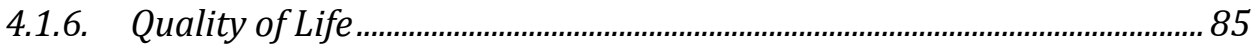

4.2. LIMITATIONS OF THE REVIEW ……………………….........................................86

\section{CHAPTER 5 CONCLUSIONS AND RECOMMENDATIONS FOR PRACTICE AND}

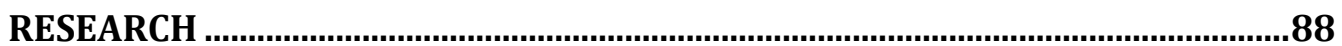

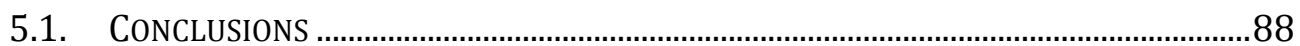

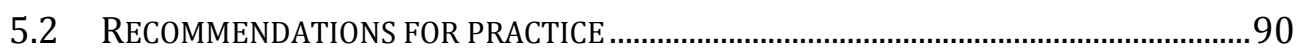

5.3 RECOMMENDATIONS FOR RESEARCH...................................................................90

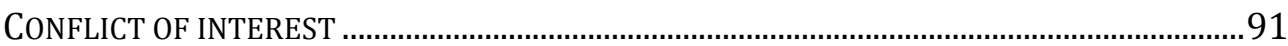

REFERENCES

APPENDICES 


\section{LIST OF TABLES}

Table 1. Results of critical appraisal of included randomised controlled trials / pseudorandomised trials . .26

Table 2. Outcome measures and corresponding studies considered in this review....30

Table 3. Studies included in the meta-analyses (Figures 2, 3 and 4) reporting on walking speed measured in metres per second ................................40

Table 4. Studies reporting stride length in metres ........................................44

Table 5. Studies included in the meta-analysis that assessed cadence measured as steps per minute ................................................................ 46

Table 6. Studies assessing step asymmetry reported as a symmetry index ............49

Table 7. Studies included assessing stance asymmetry reported as a symmetry index .51

Table 8. Studies included in a meta-analysis reporting steps per day (1000's)..........57

Table 9. Studies included in meta-analysis assessing ambulation .....................60

Table 10. Studies within the meta-analysis assessing the 'Residual Limb Health' subscale of the PEQ

Table 11. Studies included in the meta-analysis that included the Socket Comfort Score

Table 12. Studies included in narrative commentary assessing socket preference .....68

Table 13. Studies included in the meta-analysis assessing frustration ....................72

Table 14. Studies included in meta-analysis assessing utility .............................73

Table 15. Studies included in the meta-analysis assessing well-being ....................74

Table 16. Studies included in meta-analysis assessing sounds ...........................75

Table 17. Studies included in meta-analysis assessing social burden ....................76

Table 18. Studies included in the meta-analysis assessing perceived response.........77

Table 19. Studies included in the meta-analysis assessing appearance..................79 


\section{LIST OF FIGURES}

Figure 1: $\quad$ Flow chart for identification of studies for inclusion and exclusion...............24

Figure 2: $\quad$ Gait Analysis (Walking speed measured in metres / second)...................41

Figure 3: $\quad$ Gait Analysis (Walking speed measured in metres / second for socket designs with gel interfaces for TSB group and foam interface for SSB

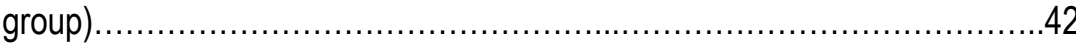

Figure 4: $\quad$ Gait Analysis (Walking speed measured in metres/second for socket designs with gel interfaces and distal suspension for TSB group and foam interface with proximal suspension for the SSB group).

Figure 5: $\quad$ Gait Analysis (Stride length measured in metres comparing the TSB and SSB socket designs). 44

Figure 6: $\quad$ Gait Analysis (Stride length measured in metres comparing the TSB with gel interfaces and distal suspension and the SSB socket design with foam interface with proximal suspension).

Figure 7: $\quad$ Gait Analysis (Cadence measured by steps per minute comparing TSB socket design with SSB socket design irrespective of interfaces and suspension methods utilised).

Figure 8: $\quad$ Gait Analysis (Cadence measured by steps per minute comparing the TSB with gel interface and distal pin suspension and SSB socket design with foam interface and proximal suspension method).

Figure 9: $\quad$ Gait Analysis (Step asymmetry assessed with a symmetry index comparing the TSB with the SSB socket design).

Figure 10: $\quad$ Gait Analysis (Stance asymmetry assessed by a symmetry index calculation comparing the TSB socket design with the SSB socket design). .51

Figure 11: Two experimental studies comparing the TSB socket design with the SSB socket design in terms of step count......

Figure 12: $\quad$ Three experimental studies comparing the TSB and SSB socket designs in terms of self-reported ambulation. .60

Figure 13: $\quad$ Three experimental studies comparing the TSB and SSB socket designs in terms of residual limb health. 
Figure 14: $\quad$ Two experimental studies comparing TSB socket design with SSB socket design in terms of socket comfort.

Figure 15: $\quad$ Socket preference (Seven experimental studies comparing TSB socket design with SSB socket design).....

Figure 16: $\quad$ Socket preference (Four experimental studies comparing the TSB with gel interface and distal pin suspension and SSB socket design with foam interface, and proximal suspension method)

Figure 17: $\quad$ Socket preference (Two experimental studies comparing the TSB with gel interface and distal pin suspension and SSB socket design with foam interface and proximal suspension method in the early rehabilitation phase).

Figure 18: $\quad$ Three experimental studies comparing TSB socket design with SSB socket design in terms of frustration with prosthesis

Figure 19: $\quad$ Two experimental studies comparing the TSB socket design with the SSB socket design in terms of utility of the prosthesis.

Figure 20: $\quad$ Two experimental studies comparing the TSB socket design with the SSB socket design in terms of self-reported well-being.

Figure 21: $\quad$ Two experimental studies comparing the TSB socket design with the SSB socket design in terms of the sounds produced by the prosthesis. .76

Figure 22: $\quad$ Two experimental studies comparing the TSB and the SSB socket designs in terms of social burden. .77

Figure 23: $\quad$ Two experimental studies comparing the TSB and the SSB socket design in terms of perceived response. 78

Figure 24: $\quad$ Two experimental studies comparing the TSB and SSB socket designs in terms of their appearance. 


\section{Abstract}

\section{Background}

Lower-limb prostheses enable life participation for people with amputation. The aim of this systematic review was to synthesise evidence on the effectiveness of total surface bearing (TSB) compared with specific surface bearing (SSB) prosthesis designs on health outcomes.

\section{Inclusion criteria}

\section{Types of participants}

Trans-tibial amputees aged 14 years and older utilising a TSB or SSB prosthesis.

\section{Types of interventions and comparators}

The intervention was the TSB and the comparator was the SSB design.

\section{Types of studies}

This review considered all relevant quantitative study designs.

\section{Outcomes and outcome measures}

Outcome measures relating to function and mobility, comfort and pain, quality of life and energy expenditure were considered.

\section{Search strategy}

A three-step search strategy across 13 databases and discipline-specific resources was pursued. Published and unpublished studies in English were considered, from database inception to June 2012.

\section{Methodological quality}

Two independent reviewers, using the Joanna Briggs Institute MAStARI appraisal checklists, undertook critical appraisal. 


\section{Data collection}

Data about interventions, populations, study methods and outcomes of significance were extracted using the MAStARI tool from the Joanna Briggs Institute.

\section{Data synthesis}

Quantitative data was pooled in statistical meta-analysis using the Cochrane Review Manager Version 5.2 where possible. Where not possible, findings were presented using narrative and tables.

\section{Results}

This review identified and analysed 28 measures assessing the health domains, presenting mixed findings. Twenty-one measures found no difference between socket designs; four found a significant difference favouring the TSB and three found a significant difference favouring the SSB design.

Suspension and interface variation was found. Sub-group analysis assessed TSB with gel interface and SSB with foam interface, to examine interface influence. Four measures found no difference and two measures, walking speed and cadence, found a significant difference favouring the TSB design.

Further sub-group analysis assessing the influence of pin suspension with TSB compared to supra-condylar suspension with SSB found significant difference favouring TSB design for walking speed and socket preference outcomes.

\section{Conclusions}

The available evidence on the effectiveness of prosthetic socket designs suggests no clear choice between the TSB and SSB. This may be due to variation in interface and suspension utilised. Interpreting the findings, the TSB was as effective as the SSB design in improving health outcomes relating to function, comfort and quality of life. 


\section{Implications for Practice}

In finding that the TSB is as effective as the SSB design in improving health outcomes implies that prescription may depend on clinician knowledge and skill-set, funding availability and patient preference.

Prosthetists require the skill-set to deliver the TSB design. TSB prescription involves a gel interface, with additional costs; therefore funding is required to enable this prescription.

\section{Implications for Research}

Additional high quality studies involving a larger sample size, across aetiologies are required. Consistency in measures is critical to facilitate comparison and enhance meta-analysis.

Studies on cost-effectiveness of socket designs are required to inform choice from a societal perspective.

\section{Keywords}

Total surface bearing, specific surface bearing, patellar tendon bearing, prosthetic, trans-tibial, amputee, systematic review 


\section{Declaration}

I certify that this work contains no material which has been accepted for the award of any other degree or diploma in my name, in any university or other tertiary institution and, to the best of my knowledge and belief, contains no material previously published or written by another person, except where due reference has been made in the text. In addition, I certify that no part of this work will, in the future, be used in a submission in my name, for any other degree or diploma in any university or other tertiary institution without the prior approval of the University of Adelaide and where applicable, any partner institution responsible for the joint-award of this degree.

I give consent to this copy of my thesis, when deposited in the University Library, being made available for loan and photocopying, subject to the provisions of the Copyright Act 1968.

I also give permission for the digital version of my thesis to be made available on the web, via the University's digital research repository, the Library Search and also through web search engines, unless permission has been granted by the University to restrict access for a period of time.

Sally Jane Cavenett:

Date: 


\section{Acknowledgments}

I extend gratitude to Dr Sarahlouise White and Dr Judith Streak Gomersall and JBI staff for their support and expertise in the development of the systematic review report, as well as Edward Ko Ko Aung MBBS for serving as the second assessor in the review.

I acknowledge and thank SA Health, Repatriation General Hospital, for providing me with study leave to undertake the critical learning coursework to enable me to commence the systematic review process.

A special thank you is reserved for my husband and children who have encouraged me, with pride and gusto to achieve this body of work, and who have allowed me the indulgence of time to complete it. Thank you to my Mother, who has assisted my family in every way possible, so that I may indulge in this process.

Most importantly, I dedicate this work in memory of Jane Hunt, my beloved Aunt, who never tired of searching for answers to her cause, to mine, and to all whom she met. A contributor and keeper of knowledge, and my inspiration for continuation of learning. 


\section{Chapter 1 Introduction}

This thesis is comprised of an application of the Joanna Briggs Institute systematic review methodology for reviewing evidence on the effectiveness of interventions (therapies, programmes or technologies). The question it addresses is what the best available evidence suggests about the relative effectiveness of two trans-tibial prosthetic socket designs, on health outcomes for people (aged 14 years and over) with amputation. The trans-tibial socket designs whose effectiveness was examined and compared were the specific surface bearing (SSB) and the total surface bearing (TSB) designs, which are most common throughout the world in prosthetic provision.

The JBI methodology for systematic review is firmly rooted in the evidence based healthcare paradigm, which sees the purpose of systematic review not only as an academic pursuit, designed to contribute to knowledge, but also as a tool to inform practice/policy and thereby enhance health outcomes. Whilst the one purpose of conducting the systematic review reported on in this thesis was to develop the knowledge base in the area and meet the requirements for the Master of Clinical Science degree, another was to provide scientific evidence that may be used by clinician's to improve their prescription formulation and thereby improve outcomes among people with limb amputation.

This first chapter of the thesis describes the context of the review (section 1.1); with reference to the existing research on prosthetics and description of socket designs, and the motivation for the research introduced (section 1.2); the purpose of the systematic review is defined (section 1.3) and an outline of the structure of the thesis is provided (section 1.4).

\subsection{Context for the review}

\subsubsection{Prosthetics}

Prosthetics is the science and art of replacing human limb segments absent from congenital cause, or amputated for reasons stemming from disease, trauma or infection. Prostheses are custom-made for every individual with fabrication commencing with a cast or digital scan of anatomical topography of the limb remnant. 
During the prescription process, consideration is required regarding prosthetic socket design and construct material used, to provide structural integrity allowing weight bearing and movement, to facilitate participation in everyday activities. ${ }^{1}$ Componentry selection relates to the modular attachment of hardware, for example, foot, ankle, lock system, and pylon in the trans-tibial prosthesis. The componentry is attached to the socket to allow for weight transfer and mobilisation.

Pre-determined factors, for example, client age, cognitive understanding, level of limb loss, body weight, skin and muscle condition can influence the prescription for both prosthetic design and componentry selection. ${ }^{2}$ Environmental influences, for example, client's geographical distance from a prosthetic facility, temperature and humidity of location, manufacturing equipment and knowledge availability of prosthetic practitioners, and cultural impediments to amputee rehabilitation can also impact on prosthetic design and prescription.3,4 Administrative factors, for example, funding availability for componentry, material and equipment can also be facilitators or inhibitors to providing prosthetic prescription options.

\subsubsection{Prosthetic prescription}

Prosthetic manufacture is commenced with a negative cast or impression taken of the individual's limb remnant (residuum), most often using plaster bandage to create the mould. The trans-tibial (or below-knee) prosthesis consists of a socket and componentry. The prosthetic socket is the surrounding encasement of the residuum and the componentry is the connecting hardware.

The increasing complexity involved in prosthetic prescription and provision, relating to the development of specific materials, software programs, and manufacturing techniques, requires the specialised knowledge of the prosthetist, to achieve best prosthetic fit for optimising mobility, comfort and function. ${ }^{1}$ Prescription indicators for componentry take into consideration patient ambulation potential and actual mobility, patient body weight, activities of daily living, recreational activities ${ }^{1}$ and funding availability.

The prescription for a prosthetic socket is often more subjective than for componentry prescription, and varies between countries, rehabilitation centres and individual 
clinicians. Clinical knowledge of the residuum surface and underlying anatomy and its physical tolerances, and the individual's ability to don and doff (put on and take off) the prosthesis are factors considered during prescription for socket design. However, if the prosthetic practitioner lacks knowledge of, or inexperience with, alternative socket designs and manufacture techniques, this limitation could be a barrier to introducing and prescribing contemporary socket designs. The cost of the residuum interface, equipment required for manufacture, the time available for service provision, or a client's capacity to readily access prosthetic service providers, may all influence prosthetic design choice.

Prosthetic replacement for the lower limb requires the prosthesis to be functional where possible to enable transfers moving from bed to chair, or weight-bearing and ambulation for participation in activities of daily living, and advanced recreational activities where desirable. The socket is a critical aspect of the prosthesis. The ability to bear weight through the prosthesis requires the socket to be manufactured with suitable structural integrity to allow weight bearing, although with enough deformation property to allow comfortable wear. An intimate fit between the socket and the residuum is crucial for the wearer's comfort, the health of the skin and tissue of the residuum, and for achieving functional outcomes with the prosthesis. ${ }^{6}$

To facilitate understanding of the results of this thesis, it is important to understand that the effectiveness of different socket designs may be confounded by factors such as the suspension method or how the prosthesis is held onto the residuum, or the type of interface liner used, the experience of the clinician fitting and/or designing the prosthesis, or the componentry utilised in the prosthetic prescription. The socket design, providing fit and resultant function, is considered the key factor influencing prosthetic use by amputee clients, regardless of componentry utilised. ${ }^{7}$

\subsubsection{Prosthetic service development}

Prescription and treatment pathways are ideally developed within a multi-disciplinary team, consisting of rehabilitation physician, prosthetist, physiotherapist, other allied health professionals and the client, to deliver prosthetic education and provide ongoing training and rehabilitation to achieve goals of participation. ${ }^{8}$ The prosthesis is designed, manufactured and fitted in a hospital or community setting by the prosthetist. 
Historically, prosthetic provision has been documented since pre-biblical times, with prosthetic development transitioning from a crude peg-leg through to stages of rehabilitative aide. ${ }^{9}$ The incidence of limb loss, and consequential need for artificial limb replacement, by necessity, accelerates during and following times of conflict. ${ }^{1}$ Seeking to determine the association between war and rehabilitation Eldar and Jelic (2003) conclude in their review of literature that rehabilitation services developed in association with wars, and the rehabilitating experience contributed to the development of principles and practices for complex impairments including amputation injuries. ${ }^{9}$

The civil war in America (1861-1865) is thought to have resulted in 300 thousand amputations. ${ }^{10}$ Prosthetic provision at this time was largely utilising raw materials of wood, leather and metal, with prosthetic manufacturers training on the job in an apprentice style pathway.

Post World War 2 (1945) professional competencies were required as prosthetists were employed within public hospital and community health sectors. Documentation of treatment designs and interventions commenced, as prosthetists were established in health facilities in response to the increasing need. ${ }^{9}$ This health care environment provided opportunities for education and information sharing, and bought about collaborative development of new techniques and componentry, and facilitated research in effectiveness and new developments.

Currently in Australia, the Master of Clinical Prosthetics and Orthotics and the Bachelor of Prosthetics and Orthotics qualifications from La Trobe University, Melbourne; provide graduates with entry level requisite for practice, and eligibility for Australian Orthotic and Prosthetic Association (AOPA) membership in Australia. ${ }^{11}$

\subsubsection{Factors that influence socket prescription and outcomes: Cause and level} of amputation, age, and componentry.

Adults may present with a trans-tibial amputation for a variety of reasons. Vascular disease, trauma, cancer, infection and congenital conditions are the leading causes for amputation of the lower-limb. ${ }^{12}$ Different countries and regions have varied numbers of amputees within each causal category.4,12 The trans-tibial prosthesis is the most commonly prescribed, as the majority of lower-limb amputees have this level of amputation irrespective of aetiology. 
Aetiology or cause of amputation can impact on health outcomes. Amputation due to disease process, for example vascular disease with or without diabetes, can often present with other co-morbidities that may influence function, ability to mobilise, motivation, and mood. Sansam and colleagues identified five studies in their systematic literature review reporting poorer outcomes relating to walking potential for dysvascular subjects than subjects of non-vascular cause. ${ }^{2}$

Amputation due to cancer typically presents in younger persons, with less if any, comorbidities. This is similar to amputees of traumatic cause, who mostly have little or no other systemic co-morbidities, although may have compromised residuum length, presence of difficult scar tissue, or unresolved pain due to nerve trauma. Studies identified in van Velzen et al's systematic review indicate that higher levels of walking ability seemed to be reached for traumatic amputees compared to that of vascular amputees. ${ }^{13}$

People utilising a prosthesis due to congenital limb absence, are often disaffected by mobility issues as they have participated all their life with a lower limb prosthesis. There is reasonably strong evidence that amputation or limb absence at a younger age leads to superior walking ability. ${ }^{2}$

Similarly, the level of the trans-tibial amputation undertaken, being the percentage of remaining tibia length in the residuum, can influence the prosthetic fit, the type of suspension required, and the amount of weight bearing tolerance that can be undertaken by the residuum. For example, a shorter length residuum requires greater surface area for weight distribution, whereby sockets are designed with an increase in the height of proximal trimlines to enhance medio-lateral knee stability. Isakov et al identified that the length of residuum influenced muscle strength, whereby subjects with shorter residuum's $(<15 \mathrm{~cm})$ had significant weaker muscles than subjects with longer residuum's. ${ }^{14}$ The longer residuum length has good musculature from gastrocnemius and soleus to allow for negative pressure systems to be utilised, with this length being more conducive to a TSB socket design or a self-suspending SSB design. The age of the amputee, cognitive ability, general fitness or activity potential can also influence mobility and participation outcomes. ${ }^{2,5,13}$

With all of these factors largely predetermined and unable to be influenced, it is accepted that consideration is given prior to prescribing a SSB or TSB, to determine 
which socket design may be appropriate for the individual. In regard to this thesis, knowing that a short residuum, or one with conical shape and prominent bony elements, excessive invagination in skin and scar tissue would not be suitable for TSB prescription; we have assumed that all authors, investigators and researchers of the included studies recruited only participants for whom it was valid to fit either a SSB, a TSB or both designs, to enable an appropriate comparison to be undertaken.

It is important to recognise that the componentry selected for use within the prosthesis may affect comfort and performance. For example, van der Linde et al (2006) investigate the effect of prosthetic components on human gait in a systematic review, and identified difference in time-distance parameters of gait in several studies when comparing prosthetic feet with different mechanical characteristics. ${ }^{15}$ Undertaking this thesis, studies have been selected where risk of bias has been reduced through consistency in use of componentry where possible. Some studies utilised feet of similar mechanical properties, and other studies reported subjects did not change their prosthetic feet from baseline to post intervention testing.

Although data is unavailable comparing number of prosthetic prescriptions specifying TSB versus SSB trans-tibial prosthetic designs in Australia or elsewhere, this thesis has sought to include as many studies as possible comparing the two socket designs regardless of reason for amputation, in order to represent the complement of amputees treated in most prosthetic clinics worldwide.

\subsubsection{Goals of prosthetic rehabilitation}

Mobility and function are often seen as primary indicators to successful rehabilitation for people with a lower limb amputation. ${ }^{2}$ The primary goals of the rehabilitation team are to provide care, education, equipment, resources and physical training that enable the client the opportunity to achieve functional independence.

The prosthesis, as an external attachment to the residuum is a piece of equipment requiring custom manufacture to assist ambulatory mobility. Ambulatory mobility in the community setting can provide the amputee with a degree of regained independence. ${ }^{16}$ Whether standing to prepare a meal, attending to self-care, returning to work or participating in high-level activities, mobility is a significant measure of ambulatory rehabilitation. 
The precise goals of rehabilitation and progressive community participation are tailored to the individuals needs and are established in collaboration with the client and the multi-disciplinary rehabilitation team; with prosthetic prescription largely determined by the prosthetist, the rehabilitation physician and the client.

1.1.6. The TSB and SSB prosthetic socket designs: Description and rationale for their selection

The total surface bearing (TSB) and specific surface bearing (SSB) socket designs were selected for consideration in this review as they represent the typical prescription options in clinical practice. Objective outcome measures are increasingly used to justify clinical prescription of prostheses through assessing the mobility, function and quality of life of people with an amputation using a prosthesis. ${ }^{17-19}$ While there is a reasonable amount of literature evaluating the effects of prosthetic componentry choice on functional outcomes for amputees, ${ }^{15}$ there is a need for a review of the evidence relating to prosthetic socket design and its possible influence on health outcomes.

A variety of materials exists including high temperature thermoplastics, acrylic and epoxy resins integrated with textiles of fiberglass, nylon, carbon-fibre and composites, to achieve the structural element surrounding the residuum which is called the prosthetic socket. The socket transfers body weight downward through the residuum to the exterior of the socket to the attachment points, which connect the socket to distal components that make up the endo-skeletal or exo-skeletal part of the prosthesis.

In circumstances of trans-femoral (above the knee) prosthetic fittings, componentry would include prosthetic knee joint, connectors, pylons in place of tibial shank, and foot/ankle componentry. With the trans-tibial (below the knee) prosthesis, the socket has an adapter connecting into a pylon or directly into the foot/ankle module.

After client residuum casting, the negative cast is usually filled with plaster to form a positive mould, with the negative cast removed once the plaster is set. Modification or rectification of the positive plaster is typically undertaken with files and fine instruments to remove plaster from the residuum copy over areas that can typically tolerate weight, and plaster is built onto the cast over areas that require relief from pressure typically being bony landmarks. 
In this thesis, a prosthetic socket produced via a rectification of prosthetic cast is referred to as the specific surface bearing (SSB) socket. This term whilst not used universally has been adopted within this thesis to represent socket designs described as Patella Tendon Bearing (PTB), Patellar Tendon Supracondular socket (PTS or PTBSC), Patellar Tendon Kegel (PTK) and Kondylen-Bein-Muenster (KBM).

Alternatively, a prosthetic socket can be designed with total surface contact and weight bearing, using the concept of evenly compressed tissue of the residuum with an enclosed or sealed system that will reduce movement within the socket, to provide a suitable socket for weight bearing, and designed to reduce the specific load onto areas of the residuum. ${ }^{20}$ This is the total surface bearing (TSB) design, which is manufactured through a uniform compression during the negative casting stage, and then a minimal amount of plaster, if any, removed globally during the rectification stage. Residuum tissue areas are not specifically loaded, and bony areas are not provided with a void as in the design of SSB rectification.

\subsubsection{The SSB socket design}

Following World War 2, the need for prosthetic provision across Europe increased markedly and provided the stimulus for the design of the SSB trans-tibial socket, commonly known as patellar tendon bearing (PTB). Described in the 1950 s by Radcliffe, the PTB socket allows persons with trans-tibial amputation to tolerate weight bearing through the residuum. ${ }^{21}$ With pressure concentrated on the tolerant patellar tendon, the posterior musculature of the gastrocnemius/soleus and medio-anteriorly on tibial flare, coupled with relief over bony prominences within the residuum, suspension of the prosthesis was achieved mostly via supracondylar cuff. ${ }^{21}$ The PTS, PTB-SC, PTK and KBM socket designs each follow a similar principle to the PTB. These designs differ slightly from the PTB design as they have additional functional effect derived from extended proximal socket trimlines forming supracondylar self-suspension, increased medio-lateral stability of the knee joint and improved cosmesis. ${ }^{22}$ The SSB socket design is an improvement on its predecessor - the 'conventional' prosthesis consisting of a proximal leather thigh corset, which allows for minimal weight to be borne through the residuum. It is heavy, cumbersome and can restrict knee joint range of motion. 
The SSB socket design is usually manufactured with a foam interface liner; however may have a silicone or gel liner within the rigid socket. The specific areas of pressure relayed to the residuum during hand casting, rectification and manufacture are unique to the SSB design, which remains in popular use throughout the world today. Beneath the foam liner, the amputee usually wears cotton or woollen sock/s directly onto the residuum; to assist with accommodating volume fluctuation and wicking away perspiration to optimise socket fit and comfort. Advantages of the SSB socket design include relatively low cost to manufacture, ${ }^{23,24}$ readily available materials for use and greater adjustability within the socket foam interface to ensure fit for the changing residuum. ${ }^{22}$

After amputation surgery, the residuum requires stabilising with oedema control, desensitisation, contracture prevention, and protection from trauma or falls; all being goals in preparation for prosthetic fitting. ${ }^{25}$ Prostheses are provided once primary healing stage has been achieved, usually 21-28 days after amputation surgery. Rehabilitation typically involves a SSB socket design to cater for the rapid and changing volume of the residuum, with alterations in both alignment and changes to the foam liner interface to accommodate changes.

Depending on service availability, hospital protocol, wound healing and general fitness and conditioning of client, prosthetic provision may be delayed. Replacement prosthetic sockets are provided to cater for changes in residuum shape and changes in client activity, throughout the rehabilitation stage and gait re-education, extending to when clients are dwelling in the community setting. Clients are likely to require ongoing prosthetic services for the remainder of their ambulatory life.

The SSB socket was defined as the comparator in the review discussed in this thesis, and the TSB socket as the intervention. We hence sought to determine if the TSB is as effective across a range of health domains for the person with a trans-tibial amputation.

\subsubsection{The TSB socket design}

In the early 1980s Ossur Kristinsson, a trans-tibial amputee and prosthetist, developed the Icelandic roll-on silicone socket (ICEROSS) liner, widely used in Iceland from 1986. ${ }^{7}$ Made from silicone and cylindrical in shape, the liner is inverted and rolls onto the residuum providing upward compression force. ${ }^{7}$ The resultant intimate fit and the nature 
of the silicone serves to provide a stabilising interface that offers the skin and tissue protection from the prosthetic socket and external forces that are transferred upward through the residuum. ${ }^{26}$ The original ICEROSS liner incorporates an attachment in the external distal end to which a corrugated pin is secured. The pin engages into a lock component fabricated into the distal end of the prosthetic socket, thus providing a secure connection between the silicone liner and the prosthetic socket, becoming the suspension mechanism.

By the 1990s, ICEROSS liners were introduced to the worldwide prosthetic community, reducing the need for supracondylar suspension for trans-tibial amputees. Advantages of using ICEROSS liners include a superior suspension system, stabilisation of soft tissue and minimal pistoning (stretching), helping to improve circulation and increased client comfort. 26

To complement the ICEROSS system, Ossur Kristinsson in 1993 described the TSB socket concept; where within the TSB socket, weight was borne by the entire surface of the residuum and the socket design was used in collaboration with the silicone liner system. ${ }^{7}$ The TSB socket design was also described by Staats, advocating the hydrostatic principle achieved by suction suspension, with or without the use of a silicone interface. ${ }^{20}$ Essentially, the TSB design relied on the hydrostatic principle of containing the entire mass of the residuum in an equal volume of socket with minimal movement/pistoning to keep the residuum in contact with the socket at all times during the gait cycle.7,20

The TSB design concept spread to clinical practice and has been reported to have had varied clinical results depending on residuum suitability, clinician knowledge, skill in fabrication and choice of the suspension system used in conjunction with the TSB design. ${ }^{23,26}$ Although at times described as a hydrostatic design, the TSB socket commonly used in practice follows the hydrostatic principle of load transfer with limited area loading introduced; for example minimal global reduction, or slight pre-tibial rectification. ${ }^{27}$ This is to cater for the changing mechanics of the residuum throughout the gait cycle.

The TSB socket design was defined in this thesis as including designs reported as TSB, hydrostatic, unrectified and limited area loading. All of these TSB socket descriptions mostly utilise a silicone or gel liner, achieving suspension through distal pin attachment, 
or a negative pressure system achieved by a sealed system secured by expulsion valve, elevated vacuum, or membrane incorporated within the interface liner with or without the use of a knee sleeve. The TSB design can be differentiated from the SSB design, as it typically lacks a foam interface liner, has no primary suspension via supracondylar means of cuff or straps and importantly has no specific increased pressure loading areas onto the residuum.

There is a dearth of evidence measuring and comparing the use of the TSB and SSB socket design types at the global level, and in Australia. Hence it is unknown what percentage of socket styles are used in clinical practice, and if this is influenced by regional area, access to equipment and materials, or training in various techniques by prosthetic practitioners.

\subsubsection{Access to prosthetic services}

\subsubsection{Funding for prostheses}

Different care models and funding opportunities influence prescription, provision and variation in prosthetic services. ${ }^{3}$ In developed countries such as Australia, access to prosthetic services including prosthetic provision and associated ambulatory rehabilitation, is provided mostly via Government funding every three years if warranted, and sooner if medically indicated.28,29 There is some variation between Australian States and Territories relating to labour costs, choice of componentry, and interface provision. ${ }^{28-30}$ Decisions regarding prescription of componentry, interface use and socket design are ideally developed and delivered in a patient-centred clinic with collaboration from prosthetist, rehabilitation physician, physiotherapist and client.

In developing or low-income countries, where funding is restricted or non-existent, prosthetic services may be accessed via non-government or not-for-profit organisations (NGO)'s usually where countries have adopted a community based rehabilitation (CBR) model.4,31 Countries and regions with limited funding for advanced componentry or interfaces, will provide traditionally designed functional prostheses, mostly SSB socket design with supracondular cuff. This design is readily adjustable, lower in cost to provide and maintain, and usually manufactured by locally trained providers whose skillset is appropriate for this manufacture technique. ${ }^{3}$ Durability, longevity, ease of construct and maintenance, culturally appropriate design and cosmesis, are key 
considerations of prosthetic provision. It is widely thought that prostheses which have high durability and delivered at low cost, should be the first choice for individuals in developing regions. ${ }^{3,32}$

In underdeveloped countries where NGO's are limited, the demand for prosthetic service often outstrips available facilities, staff and training, and access to materials. ${ }^{3}$ Therefore ambulation mobility is via mobility aids such as crutches or wheelchairs or rudimentary devices when prosthetic services are unavailable or inaccessible.

\subsubsection{Conceptualisation of outcomes}

The use of domains relating to health can be somewhat complex, depending on the area of interest and interpretation. The scope and classification of outcomes in this thesis was informed by the World Health Organisation's (WHO) international classification of functioning disability and health (ICF). ${ }^{33}$ The ICF identifies healthrelated domains for measuring the effects of health, both negative and positive, on any given disease, diagnosis or disability group. These domains are classified from body, individual and societal perspectives by means of two lists: i) body functions and structures, and ii) activities and participation. 33

The health outcomes and measures identified in this thesis were typically used in clinical practice in hospital, rehabilitation and community settings; for example, Step Activity Monitor, ${ }^{34}$ Prosthetic Evaluation Questionnaire, ${ }^{35}$ and the Socket Comfort Score $; 6$ to assist clinicians and prescribers regarding the function of the prosthesis provided. A range of measures, subjective and objective, were employed throughout the included studies.

Irrespective of the reasons for prescription, there is a need for a greater understanding of the effect of using different socket designs in terms of client rehabilitation, mobility, function and quality of life; to ensure that socket design is thoroughly considered during the prescription process and the socket that is prescribed maximises the clients wellbeing.

\subsection{Motivation for the research: The value of the systematic review}

Practitioners, service managers and clients need to be kept informed about new technologies, techniques and interventions that can influence health outcomes for 
prosthetic users. Clinical decision-making often falls to practitioner experience and expert opinion. Rarely are the resources, time and skill available to review, appraise and interpret all research-based evidence. Therefore, the use of synthesised research findings in the form of a systematic review can make this task manageable. A systematic review seeks to collate and critically appraise all research evidence that fits a pre-specified criteria in order to address a specific research question. ${ }^{37}$

As the technique of socket manufacture has progressed over the last two decades, a number of studies have compared the traditional SSB design with the more contemporary TSB design. Several reviews2,13,15,18,32,38 have been conducted relating to prosthetic components and suspension systems effect within a prosthesis; or regarding outcomes of quality of life, potential ambulation or functional mobility with a lower limb prosthesis. These reviews have not examined effectiveness of TSB socket design compared to SSB socket design.

The following systematic reviews were identified during the scoping process prior to conducting the systematic review discussed in this thesis:

i) van der Linde et al15 published "A systematic review of the effect of different prosthetic components on human functioning with a lower limb prosthesis" in 2004. This review included all levels of lower limb amputation, with focus on prosthetic feet, prosthetic knees, prosthetic sockets and prosthetic mass. Only one socket study was identified which did not compare TSB design to SSB design. This review provides us with insight as to differences in effect with different components, therefore emphasises the importance of utilising consistent componentry within studies.

ii) In 2006 van Velzen ${ }^{13}$ published "Physical capacity and walking ability after lower limb amputation: a systematic review". All levels of lower limb amputation were included, without reference to socket design utilised. This review provided us with insight as to the relationship between walking capacity and walking ability, and broadens our understanding of the influence of cause of amputation on walking ability.

iii) Sansam and colleagues ${ }^{2}$ published "Predicting walking ability following lower limb amputation: A systematic review of the literature" in 2009. This review included all levels of lower limb amputation, without reference to socket design. It contributes to our knowledge surrounding health conditions, physical fitness, balance and standing ability, 
and how personal contextual factors can contribute to an individual's mobility after lower limb loss.

iv) Authors Sinha and Van den Heuvel ${ }^{38}$ conducted a systematic review in 2011 "A Systematic review of quality of life in lower limb amputees". Whilst this was one of the domains we identified to include in our systematic review, the authors largely excluded sources of grey literature, included lower limb amputees of all levels, with the review not being specific to trans-tibial socket design.

v) In 2011 Jowen Penn-Barwell18 published "Outcomes in lower limb amputation following trauma: A systematic review and meta-analysis". The author included only traumatic amputees, of various lower limb levels and pooled data relating to quality of life, mobility, employment, prosthesis use and pain symptoms. Whilst outcomes were the primary focus of this review, socket design was not identified or discussed.

vi) Gholizadeh et al32 published "Transtibial prosthesis suspension systems: Systematic review of literature" in 2013. This review focused on the various suspension systems utilised with a trans-tibial prostheses, with some apparent cross-over with our study. The main difference identified is that Gholizadeh and colleagues investigated suspension system effectiveness and not socket design effectiveness. This review broadens our understanding as to the effectiveness of a suspension system that is designed to reduce pistoning during mobilising.

In order to gain a better understanding of the contributing factors that influence health outcomes for prosthetic users, a review of socket design is required. To inform practise, a review of all relevant evidence needs to be undertaken to collate the results in a usable format. The development of evidence based clinical guidelines can assist practitioners in determining when to prescribe a particular type of prosthetic socket, and for whom it would be of most benefit, and under what circumstances of health. A systematic review is the first step in guideline development and is a tool for improving health outcomes, by combining outcomes of primary studies, and also identifying gaps in existing evidence. ${ }^{37}$ 


\subsection{Systematic review and evidence based healthcare}

Reviews of evidence seek to collate and synthesise evidence on a particular subject. There are a range of review types referred to in evidence-based practise, with the literature review, the scoping review and systematic reviews amongst the fourteen review types identified by Grant and Booth (2009). ${ }^{39}$ A description of these common reviews is outlined.

A literature review generally includes published material that provide examination of recent or current literature, may or may not include comprehensive searching, may or may not include a quality appraisal assessment, the evidence synthesis is typically narrative, and analysis may be chronological, conceptual or thematic. ${ }^{39}$

A scoping review is often used to inform systematic reviews by identifying research gaps and summarising findings of research. ${ }^{40}$ Scoping reviews identify a research question and relevant studies, though do not include a quality assessment of included studies, which limits data synthesis and interpretation. ${ }^{40}$ Data is usually charted and summarised, and can usually be conducted in a short time frame.

A systematic review is a process of transparent, robust methodology whereby researchers can define a research question, conduct an extensive search, identify all primary research relating to an area of investigation, and determine the rigor of those studies via critical appraisal using standardised appraisal tools for methodological quality. 39,40 Upon determining primary research both published and unpublished; interventions, comparators and outcomes are examined to identify research that can be appropriately combined to enable the results of similar studies to be pooled where possible to create a larger body of work. The analysis in the review process investigates if combined results provide a more meaningful interpretation of outcome, simply due to the larger power of participant numbers.

Systematic reviews may consider different types of evidence; quantitative, qualitative, an evaluation of health economics, or textual evidence. ${ }^{41} \mathrm{~A}$ comprehensive systematic review, is a review that considers two or more types of evidence. ${ }^{41}$

Systematic reviews differ from literature, narrative and scoping reviews by adhering to guidelines on the conduct of a review ${ }^{39}$ involving the following seven steps: 
1. Research Question: The first step in performing a systematic review is to formulate a primary research question as part of the research protocol which encapsulates the objective of the review. ${ }^{41}$ The research question identifies the inclusion criteria for considering studies and should include reference to the review's intended population, intervention, comparator, and outcomes (PICO). ${ }^{41}$

2. Research Protocol: Once the research question is formulated, the research protocol is developed. The goal of developing a research protocol is to develop formulation of the questions and methods of the review before retrieving the literature. The methods for literature searching, screening, data extraction, and analysis should be contained in the protocol to minimise bias before starting the literature search. ${ }^{41} \mathrm{~A}$ requirement of a Joanna Briggs Institute $(\mathrm{JBI})$ systematic review process is for an a priori published protocol. 42

3. Comprehensive search strategy: The literature search aims for exhaustive, comprehensive searching 39 to identify all international research relating to the review question. ${ }^{41}$ The search strategies aims to identify both published and unpublished studies utilising a three stage search process. ${ }^{41}$

4. Critical Appraisal: A systematic review aims to synthesise the best available evidence, therefore the methodological quality of included studies needs to appraised using validated checklist or tool to assess for biases. ${ }^{41}$ This quality assessment is undertaken by two reviewers to determine inclusion/exclusion of studies. ${ }^{39}$

5. Data Extraction: Details regarding participants, interventions, comparators and outcomes are to be extracted from included studies. ${ }^{41}$ Use of a standardised extraction tool aims to minimise errors in extracting data.

6. Data Synthesis: After including and excluding studies based on the quality appraisal, data analysis of results can be undertaken. It is important to combine study data only when it is appropriate to do so, otherwise analysis and subsequent conclusions drawn may not be valid. ${ }^{41}$

7. Interpretation of Results: This information can largely be presented in the data analysis and results table in the manuscript. The strengths and weaknesses of the included studies must be discussed. Conclusions should be based on the best available scientific evidence. Recommendations for practice and future research can be made. ${ }^{39}$ 
Care is needed to examine the methodology of published reviews when using their outcome to inform practice, as some reviews that may identify as a 'systematic review', may not have adhered to the conduct guidelines.

Systematic reviews were first utilised within clinical psychology and described by Smith and Glass et al in 1980,43 and later described by Professor David Sackett relating to evidence based medicine as 'the conscientious, explicit, judicious use of current best evidence in making decisions about the care of individual patients. '44

In a research context, the systematic review is considered to be the study design with the highest level of study quality, ${ }^{37}$ and often cited when decisions for health practice and implementation are undertaken. Common methodologies for systematic review process within healthcare are the Cochrane Collaboration, ${ }^{37}$ and the Joanna Briggs Institute (JBI) models. ${ }^{41}$

The JBI model of evidence-based healthcare seeks to include published and unpublished studies including dissertations, conference proceedings or wider expert opinion; to provide a comprehensive platform for identifying relevant research that may be considered for systematic review inclusion. ${ }^{41}$ The JBI model of systematic reviews sits within the institute's framework for translating evidence into practice which involves disseminating, implementing and evaluating evidence based guidelines in clinical settings, as well as examining the scientific and professional literature. 45

In this thesis, and the systematic review that underpins it, the effectiveness of total surface bearing compared to specific surface bearing prosthetic socket design was examined on selected health outcomes of adults with a trans-tibial amputation using JBI methodology.

\subsection{Overarching research objective}

The purpose of the systematic review based research was to identify, assess, synthesise and present the best available evidence on the effectiveness of the TSB socket design compared with the SSB socket design on health outcomes of function, mobility, comfort and pain, energy expenditure and prosthetic and health-related quality of life of adults and adolescents with a trans-tibial amputation; and to assess evidence 
irrespective of interface or suspension utilised, irrespective of cause, level of amputation, or years since amputation.

The question the systematic review addressed, and this thesis focuses on is whether the effects of the contemporary total surface bearing (TSB) socket design on these outcomes outweighed those of the more traditional specific surface bearing (SSB) socket design.

\subsubsection{Structure of dissertation}

This dissertation is organised into seven chapters. This review dissertation consists of:

Chapter 1: Introduction: The first chapter describes the context of the review with reference to the existing research on prosthetics and description of socket designs. Motivation for the research is introduced and the purpose of the systematic review is defined.

Chapter 2: Systematic review methods: The second chapter describes the methodological process undertaken in the systematic review underpinning this dissertation. This chapter describes the types of studies, participants, types of intervention and comparator, and the outcomes and outcome measures utilised in the studies. The search strategy is detailed, alongside the appraisal process for methodological quality, the process of data extraction undertaken and the method of data synthesis.

Chapter 3: Results: The third chapter describes search results, the methodological quality and study characteristics of included studies. The findings of the review and the synthesised findings are presented.

Chapter 4: Discussion: The fourth chapter discusses the main findings from the data extracted from systematic review included studies, and the limitations within the review.

Chapter 5: Conclusions and recommendations for practice and research: The fifth chapter describes the conclusions, the implications for research and the implications for practice drawn from the results of the systematic review.

Chapter 6: References: The sixth chapter details references used throughout the thesis. Chapter 7: Appendices: The final chapter presents the nine appendices referred to throughout the dissertation. 


\section{Chapter 2 Systematic Review Methods}

Chapter 2 outlines the systematic review methods. This covers the review eligibility criteria, search strategy, study selection process including how studies were critically appraised, the data extraction and data synthesis methods. The a priori systematic review protocol is presented in Appendix I.

\subsection{Types of studies}

The review, on which this thesis reports, considered both experimental and observational study designs, including randomised controlled trials, non-randomised controlled trials, quasi-experimental studies, before and after studies, prospective and retrospective cohort studies, case control studies and analytical cross sectional studies for inclusion. It also considered descriptive studies including case series for inclusion, as long as participants had utilised both the TSB and SSB socket designs within the study.

\subsection{Types of participants}

This review included adult and adolescents aged 14 years and older, with acquired or congenital limb loss at the trans-tibial level, irrespective of the aetiology, time since amputation or the presence of co-morbidities, using prostheses with either the contemporary TSB socket design or the traditional SSB socket design. To be considered for inclusion, studies had to have documented study populations utilising both the TSB and SSB socket designs within the study.

\subsection{Types of interventions and comparators}

The intervention of interest was the use of the TSB socket. This is the 'hydrostatic' or 'limited load bearing' design often using a silicone or gel liner interface, with suspension achieved via distal attachment of a pin and lock, or via a negative pressure system using an expulsion valve and sleeve, vacuum suction, integrated membrane seal or a combination of these. 
The comparative intervention was the SSB socket design. This is often referred to as a PTB, PTS, PTB-SC, PTK or KBM and typically utilises a soft foam liner interface paired with supracondylar suspension.

Variation existed in the suspension and interface used, requiring sub-group analyses to show the effects of interface and/or suspension, if any, on health outcomes.

\subsection{Types of outcomes and outcome measures}

This review considered studies that included at least one of the following outcomes:

- Function and Mobility: assessed using physical measures including laboratory-based gait analysis, community-based Step Activity Monitors, ${ }^{34}$ or self-report sub-scales of the Prosthesis Evaluation Questionnaire $(\mathrm{PEQ})^{35}$ relating to function and ambulation.

- Comfort and Pain: measured using Questionnaires such as the Socket Comfort Score (SCS), ${ }^{36}$ Brief Pain Inventory (BPI), ${ }^{46}$ or sub-scale of the PEQ.

- Quality of Life: measured using questionnaires such as the sub-scales of the PEQ35 and self-reported preference for socket design.

- Energy Expenditure: measured using a physical measure of oxygen uptake during a graded exercise test.

\subsection{Search Strategy}

The search strategy aimed to find both published and unpublished studies. A three-step search strategy was utilised. An initial limited search of PubMED, Scopus and CINAHL was undertaken, followed by analysis of the text words contained in the title and abstract and of the index terms used to describe each article. A second search using all identified keywords and index terms was then undertaken across all included databases. Thirdly, the reference lists of all identified reports and articles were searched for additional studies. The search strategies are appended (Appendix II).

Only studies published in the English language were considered for inclusion. Studies published from the inception of the included databases until the $30^{\text {th }}$ of June 2012 were considered.

The databases searched were: 
PubMED

CINAHL Plus

Scopus

Embase

PEDro

Mednar

Cochrane Central Register of Controlled Trials (CENTRAL)

ProQuest Dissertations and Theses

National Library of Australia's Trove service

Electronic Thesis Online Service (EThOS)

Australian New Zealand Clinical Trial Registry (ANZCTR)

EU Clinical Trials Registry (EU-CTR)

Clinical Trials.gov - USA Trial Registry

The websites, gazettes, conference and congress proceedings of the professional bodies of the International Society of Prosthetics and Orthotics (ISPO), the Australian Orthotic and Prosthetic Association Inc. (AOPA) and the American Academy of Orthotics and Prosthetics (AAOP), and Conference Proceedings Citation Indexes (Web of Science) were explored for unpublished studies.

Initial keywords were:

'TSB', 'SSB', 'PTB', 'total surface bearing', 'specific surface bearing', 'patellar tendon bearing', 'prosthetic', 'functional outcomes', 'socket design', 'amputee', 'trans-tibial' 'outcome measures', 'domains', 'lower-limb'.

\subsection{Assessment of methodological quality and approach to study inclusion}

Papers selected for retrieval were assessed by two independent reviewers for methodological validity prior to inclusion in the review using standardised critical appraisal instruments from the Joanna Briggs Institute Meta-Analysis of Statistics 
Assessment and Review Instrument (JBI-MAStARI) (Appendix III). No disagreements arose between the reviewers regarding inclusion of papers.

\subsection{Data extraction}

Data was extracted from papers included in the review using the standardised data extraction tool from JBI-MAStARI (Appendix IV). The data extracted included details about the interventions, populations, study methods and outcomes of significance to the review question and specific objectives. The extracted data was used to populate characteristics of included studies table (Appendix VII).

\subsection{Data synthesis}

Quantitative data was pooled in statistical meta-analysis using Cochrane Review Manager version 5.2, where appropriate. ${ }^{47}$ Results were subject to double data entry. Effect sizes expressed as odds ratios (for categorical data) and weighted mean differences (for continuous data) and their 95\% confidence intervals were calculated for analysis. Heterogeneity was assessed statistically using the standard chi-square test with fixed effects for all meta-analyses. However, due to the estimated variation in participant aetiology, unstated co-morbidities, the interface and suspension systems utilised and the influence of componentry across studies, analyses were repeated using a random effects model and these are the findings reported in this thesis. Where statistical pooling was not possible, the findings are presented in narrative form.

Due to the variation in the suspension modes and interfaces used in the included studies, sub-group analyses were undertaken to examine possible influence of the type of interface and/or suspension on the size and direction of the measures of effect. 


\section{Chapter 3 Results}

\subsection{Search results}

The search results and study selection process is outlined in Figure 1. The 13 databases and sources of unpublished literature yielded 864 articles for review, of which 544 were obvious exclusions as they related to dentistry or joint replacement, for example. A further 99 titles were duplicate publications, leaving 221 abstracts that were examined for relevance to the review based on title and abstract content. After scrutinising on the basis of the inclusion criteria, 25 abstracts were retained with full articles retrieved. On review of the references for these articles, another citation was added. Therefore, 26 articles were retrieved in full text for detailed examination, as illustrated in Appendix V.

A further 14 articles were excluded after full text examination, leaving 12 articles to undergo critical appraisal. As described in section 2.5.2, the tools used to conduct critical appraisal are shown in Appendix III. One article was split into two, as it had two clear sub-populations meeting all inclusion criteria, with one population group from the inpatient setting and the other from the outpatient setting. ${ }^{48}$ Splitting the total study population into two, Hachisuka $A,{ }^{48 a}$ and Hachisuka $B,{ }^{48 b}$ was primarily for ease of data analysis. Critical appraisal was re-done for this article. Therefore a total of 13 articles underwent critical appraisal. Two studies were excluded at the critical appraisal stage due to poor methodological quality.49,27 Appendix VI provides details of the excluded papers and reasons for their exclusion. The characteristics of the 11 articles included in the review are presented in Appendix VII. 


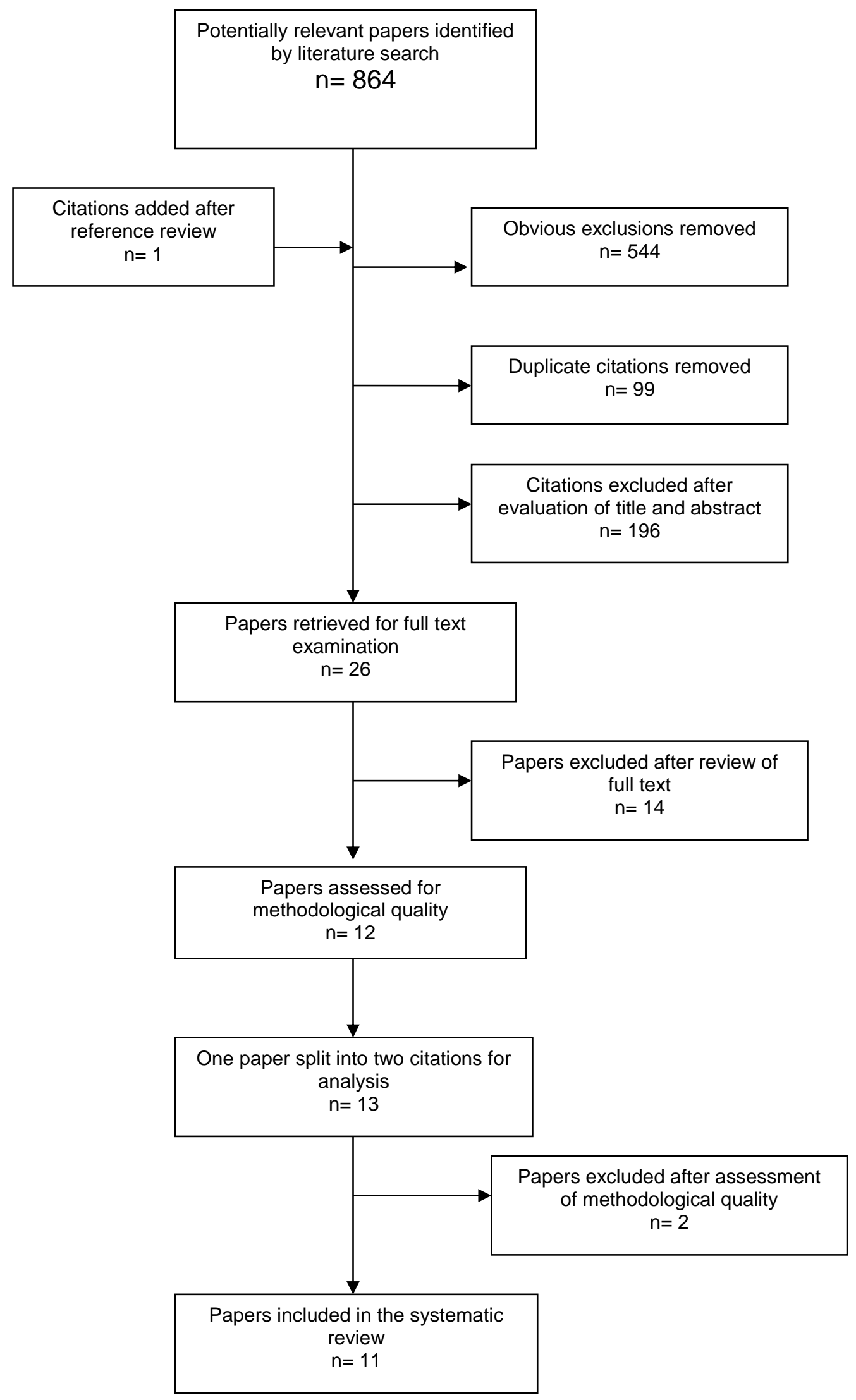

Figure 1: Flow chart for identification of studies for inclusion and exclusion 


\subsection{Methodological quality}

Following the search process, 13 studies were critically appraised by two independent reviewers to assess their methodological quality and subsequently include or exclude them from this review. No disagreements arose between the reviewers during the critical appraisal process. Both reviewers agreed that two of the studies were not of satisfactory methodological quality and should be excluded. The main reason these two studies were not included was due to the lack of relevant outcome measures and limited acclimatisation to socket design in the methodology (Appendix VI).

Of the 11 included studies, 10 were experimental in design, of which five were randomised controlled trials $23,24,50,51,52$ one was a pseudo-randomised controlled trial design ${ }^{53}$ and four were quasi-experimental before-and-after studies. ${ }^{48 a, 48 b, 54,55} \mathrm{~A}$ single descriptive observational study of case series design was the remaining included study. .56

The results of critical appraisal are presented in Table 1 for the experimental studies and in narration for the descriptive study. Corresponding questions can be found in Appendix III. Overall, the methodological quality of the randomised and pseudorandomised controlled trials was high, although for questions 1, 2, 3 and 5 (questions related to randomisation, allocation blinding and allocation concealment) of the quality appraisal checklist, there was no or little evidence of these procedures (Table 1). For question 2, this is understandable as clinicians and researchers were unable to blind the patient to the interventions received in this review and in question 5 , because concealment of socket design is difficult when collecting functional outcome data. Additionally, outcomes were deemed to have been measured with objective criteria if they were not self-reported, or able to be influenced by the researcher, therefore this thesis did not consider a negative response for these questions to be fatal methodological flaws.

Studies were assessed for risk of selection bias, performance bias, attrition bias and detection bias using the standardised JBI critical appraisal tool. Across the included studies attrition was very low. Overall, eight studies met five or more criteria, indicating sufficient methodological quality for inclusion. 
Table 1: Results of critical appraisal of included Randomised Controlled Trials / Pseudo-randomised Trials

\begin{tabular}{|l|l|l|l|l|l|l|l|l|l|l|}
\hline Citation & Q1 & Q2 & Q3 & Q4 & Q5 & Q6 & Q7 & Q8 & Q9 & Q10 \\
\hline Coleman 200423 & Y & N/A & U & Y & U & Y & Y & Y & Y & Y \\
\hline Datta 200424 & Y & N/A & N & Y & U & Y & Y & Y & Y & Y \\
\hline Hachisuka A199848a & N & N & N & U & U & Y & Y & Y & N & N/A \\
\hline Hachisuka B 199848b & N & N & U & Y & U & Y & Y & Y & Y & N \\
\hline Engsberg 200650 & Y & N/A & N & N/A & Y & Y & Y & Y & Y & Y \\
\hline Manucharian 201153 & N/A & N/A & U & N/A & U & Y & Y & Y & Y & Y \\
\hline Klute 201151 & Y & N/A & N & Y & N & Y & Y & Y & Y & Y \\
\hline Selles 200552 & Y & N/A & U & Y & U & Y & Y & Y & Y & Y \\
\hline Astrom 200454 & N & N & U & N/A & Y & Y & Y & Y & Y & N \\
\hline Yigiter 200255 & N & N/A & N & N/A & N & N/A & Y & Y & Y & Y \\
\hline
\end{tabular}

Abbreviation: $Q=$ Question; $Y=$ Yes; N=No; U=Unclear; N/A=Not applicable

Datta et al. 1996 was the only descriptive case series study included in the review. ${ }^{56}$ The study met six of the nine quality assessment criteria set in the JBI critical appraisal tool for this study design type and hence may be considered as a relatively high quality case series study. Regarding the shortcomings in study design, a random sample was not obtained, the outcome measure of patient preference for socket design was subjective in nature delivered via a self-report questionnaire and there was not a control group for comparison described.

Of the included 11 studies; using the JBI classification system for ranking evidence on effectiveness, 10 may be classified as level 2 primary studies of effectiveness and one is a level 3 study of effectiveness (Appendix IX).

\subsection{Search study characteristics}

\subsubsection{Study settings}

Of the 11 included studies, all were written in English. There was wide variety in the geographical location where the studies were conducted and within the populations studied. Four studies were conducted in the USA, $23,50,51,52$ two in the UK, ${ }^{24,56}$ and one 
each in the Netherlands, ${ }^{52}$ Sweden, ${ }^{54}$ and Turkey, ${ }^{55}$ and one conducted in Japan. ${ }^{48}$ The latter was the study which was split into two studies for ease of analysis. ${ }^{48}$

Eight studies were conducted with subjects recruited from a hospital outpatient setting with a prosthetic facility; $23,24,48 a, 50,52,53,54,56$ two studies were conducted with subjects in the inpatient rehabilitation hospital setting, ${ }^{48 b, 55}$ and one study did not define the study setting. ${ }^{51}$

Studies were published between the years 1996 and 2011.

\subsubsection{Interventions and comparators}

All studies reported using an intervention of a TSB socket design and comparator of SSB socket design, however there was variation in the description of the actual socket. Five authors were contacted for clarification of socket design terminology, with confirmation received from Coleman and Engsberg prior to inclusion. ${ }^{23,50}$ Attempts were made to contact Astrom et al. to confirm pre-existing socket design with a sample of 29 subjects; however without receiving confirmation, the decision was made to utilise results only from the seven participants in a gait sub-group, where SSB was stated as the previously used design and TSB as the intervention. ${ }^{54}$.Datta et al. were contacted to confirm the TSB design was used with a gel interface system, outlined with TSB terminology. ${ }^{24}$ Without receiving a response, the study was included as the technique described and description of the product used with ICEROSS liner was consistent with a TSB socket design technique in the gel interface group. Yigiter's study of 20 subjects was also included, as the paper specified that a TSB socket was used; however it was assumed with a gel interface, and SSB socket with assumed foam interface. ${ }^{55}$ These assumptions were based on an inference made in the paper to this effect.

With respect to the nature of the intervention, six studies used TSB sockets with a gel interface combined with distal pin lock suspension;23,24,48a,48b,52,56 one used a gel interface with vacuum suspension; 51 one used a gel interface with sleeve and valve suspension; 54 one used a foam interface with proximal sleeve suspension; ${ }^{53}$ and two studies did not specify the interface or suspension method used with the TSB design. 50,55

Regarding the comparator, six studies used a foam interface with proximal supracondylar suspension $24,48 a, 48 b, 50,55,56$ two studies used a foam interface with proximal sleeve suspension 23,53 two studies used a gel interface with distal pin lock 
suspension ${ }^{51,52}$ and one study used a gel interface for some subjects and foam interface for others, all using supracondylar suspension. ${ }^{54}$

\subsubsection{Participants}

\subsubsection{Age}

All studies included adults and/or adolescents with unilateral trans-tibial amputation, with a mean age across all included studies of 47.6 years (range: 14-81 years). One study reported participant age in categories of under 40 years, 40-49 years, 50-59 years, $60-69$ years and 70 years or older, without specifying the mean age or range. ${ }^{53}$ The study by Yigiter was noticeably different from the other included studies in terms of participant age, with a significantly younger study population (mean age of $27.8+/-7.0$ years). 55

Participant numbers were relatively low across the 11 included studies, with a mean number of participants of 23 participants across the included studies (range 5 - 54), and combined total number of participants in the review of 257.

\subsubsection{Co-morbidities, baseline activity, and years since amputation}

Nine of the included studies reported a population with no other significant comorbidities at the time of the study. 23,24,48a,50-54,56 Participants in these studies had been wearing a prosthesis for a minimum of one year and were walking unassisted in the community, equating to a functional ambulation minimum K-level of K2.57 Seven of these nine studies reported participants as having a mean of 14.7 years since amputation, 23,24,48a,50-52,54 however two studies did not report on the number of years since amputation. 53,56 Two studies recruited new amputees undergoing post-amputation rehabilitation and who were yet to receive their first prosthesis. ${ }^{48 b, 55}$

\subsubsection{Aetiology / cause of amputation}

Aetiology was reported by 10 studies.23,24,48a,48b,51-56 The most commonly occurring causes of amputation across these studies were as follows: $53 \%$ trauma, $34 \%$ dysvascular (with or without diabetes) and other causes including congenital, infection, neoplastic and miscellaneous accounting for $13 \%$ of amputations. The studies by Coleman and Yigiter were the only studies included in this review to include a single aetiology or cause of amputation, identified as 'trauma' in their studies. ${ }^{23,55}$ 


\subsubsection{Follow-up in measurement of outcomes}

The duration of follow-up is indicated by the time for acclimatisation on each socket design prior to assessment of its impact on the outcome measures. The acclimatisation period ranged from 10 days to 104 weeks. In the rehabilitation setting with new amputees, acclimatisation ranged from 10 days to eight weeks. ${ }^{486,55}$ In the experimental studies with established amputee participants, acclimatisation ranged from three to 12 weeks. ${ }^{23,24,48 a, 50-54}$ In a descriptive case series study of established amputee participants, acclimatisation ranged from two weeks to 104 weeks. ${ }^{56}$

Clinical practice would typically undertake three weeks minimum acclimatisation when assessing socket change prescription, and six weeks minimum when assessing prosthetic componentry changes.

\subsection{Findings of the review}

The presentation of findings is organised in three parts as follows. First, the range of outcomes and instruments covered in the analysis is described. This is followed by a brief description of the individual studies on which the synthesis is based. Third, the synthesis method and findings are presented. Readers are referred to Appendix VII 'Characteristics of Included Studies' and Appendix VIII 'List of study findings' for a quick overview of the nature of included studies and findings.

\subsubsection{Reported outcomes and measurement scales/instruments}

The outcomes identified and included were assessed in the primary studies using both subjective and objective measures relating to function and mobility, comfort and pain, quality of life and energy expenditure. Whilst measures of prosthetic mass and economic variables (cost of materials and fabrication for prosthetic manufacture) were identified in some included studies, they were not extracted and analysed as this was beyond the defined scope of the review. As evidence on cost effectiveness is important for directing efficient decision-making regarding choice of socket design looking ahead, the issue of cost is returned to in the discussion and conclusion of the review.

Table 2 details the identified outcomes common to all 11 studies, relating to the health outcomes of interest and the instruments used to measure these. Outcome measures used in the included studies are discussed in full within the synthesised findings section (3.5.). 
Table 2: Outcome measures and corresponding studies considered in this review

\begin{tabular}{|c|c|c|c|c|c|}
\hline Domain & Outcome & Setting/scale & Reliability & Citation & Data \\
\hline \multirow{12}{*}{$\begin{array}{l}\text { Function \& } \\
\text { mobility }\end{array}$} & Walk speed & \multirow{6}{*}{$\begin{array}{l}\text { Laboratory based } \\
\text { Gait Analysis }\end{array}$} & Objective & $24,50,52,54,55$ & M-A \\
\hline & Stride length & & Objective & $23,24,50,52,55$ & M-A \\
\hline & Step width & & Objective & 55 & Narrative \\
\hline & Temporal asymmetry & & Objective & 52,55 & M-A \\
\hline & Spatial asymmetry & & Objective & 50,52 & M-A \\
\hline & Cadence & & Objective & $24,50,55$ & M-A \\
\hline & Balance & Laboratory based & Objective & 55 & Narrative \\
\hline & Weight bearing & Laboratory based & Objective & 55 & Narrative \\
\hline & Activities of daily living & \multirow[b]{3}{*}{$\begin{array}{l}\text { Laboratory \& } \\
\text { community based }\end{array}$} & Objective & 52,55 & Narrative \\
\hline & Step Count & & Objective & 23,51 & M-A \\
\hline & Wear time & & Objective & 23 & Narrative \\
\hline & Ambulation & PEQ subscale & Validated & $23,50,51$ & M-A \\
\hline \multirow[t]{6}{*}{ Pain/comfort } & Socket Comfort Score & \multirow{3}{*}{ Questionnaire } & Validated & 23,53 & $M-A$ \\
\hline & Brief Pain Inventory & & Validated & 23 & Narrative \\
\hline & Wear comfort & & Non-validated & 56 & Narrative \\
\hline & Residual Limb Health & \multirow[t]{3}{*}{ PEQ subscale } & Validated & $23,50,51$ & M-A \\
\hline & Pain & & Non-Validated & 52 & Narrative \\
\hline & Phantom Pain & & Non-Validated & 52 & Narrative \\
\hline \multirow{9}{*}{$\begin{array}{l}\text { Quality of } \\
\text { life }\end{array}$} & Socket preference & Self-report & Non-validated & $23,48 a, 48 b, 50,52,55$ & M-A \\
\hline & Frustration & \multirow{8}{*}{ PEQ subscale } & Validated & $23,50,52$ & M-A \\
\hline & Utility & & Validated & 23,50 & M-A \\
\hline & Well-being & & Validated & 23,50 & $M-A$ \\
\hline & Sounds & & Validated & 23,50 & $M-A$ \\
\hline & Social Burden & & Validated & 23,50 & M-A \\
\hline & Perceived Response & & Validated & 23,50 & $M-A$ \\
\hline & Appearance & & Validated & 23,50 & $M-A$ \\
\hline & Satisfaction & & Non-validated & 52 & Narrative \\
\hline Energy use & $\mathrm{O}_{2}$ Uptake & Exercise test & Objective & 50 & Narrative \\
\hline
\end{tabular}

Abbreviation: PEQ: Prosthesis Evaluation Questionnaire; M-A: Meta-analysis 
Overall, 28 outcomes were identified and assessed from the 11 studies. As already outlined above and will be seen below, it was appropriate to combine only some of the measures using meta-analysis.

\subsubsection{Characteristics of individual studies}

Coleman et al., $2004^{23}$

This RCT with cross-over design compares prosthetic outcomes using gel interface and pin lock system, with those that use a foam interface with sleeve suspension. The author confirmed that the gel system was a TSB socket design and the foam interface was an SSB socket design.

Thirteen subjects completed the study, with the authors asserting in their hypotheses that ambulatory activity, prosthetic wear time, comfort and satisfaction would increase with the TSB socket design with gel liner and pin suspension system. Two and a half months acclimatisation was given with each socket design, whereby all subjects received new sockets of both the SSB and TSB design, regardless of the system they were using at the time of recruitment. Although participants were asked to wear the newly provided prosthesis throughout the acclimatisation phase and the testing period, participants had access to their previous prosthesis; therefore sole use of study prosthesis was not ensured.

The subjects recruited had amputations as a consequence of trauma, with a mean age of 49 years (range: 31.5 - 65.8 years of age), with an average of 24 years since amputation (range: 4.7 - 39.3 years). Activity levels were assessed using the Amputee Mobility Predictor tool (AMPRO). The AMPRO provides a score that relates to the USA Medicare functional levels. ${ }^{57} \mathrm{~K}$-levels range from $\mathrm{KO}$ to $\mathrm{K} 4$ and are widely used in prosthetic rehabilitation and prescription in Australia and the USA. Participant activity levels were as follows: one subject $=\mathrm{K} 2$, eight subjects $=\mathrm{K} 3$ and four subjects $=\mathrm{K} 4$.

Function and mobility was measured with a step-activity monitor, worn in the subject's everyday life setting for a consecutive two-week period. The data recorded included steps per minute and overall wear time while the monitor is attached to the participant's study prosthesis. The impact of socket design on quality of life was investigated using seven subscales of the PEQ. ${ }^{35}$

Datta et al., $2004^{24}$ 
This RCT compared gait characteristics using the PTB SSB sockets with gel interface and the TSB socket design for trans-tibial amputees. Data was collected from 21 amputees, with a mean age of 51.7 years $(S D=15$; range $=14.3-79$ years $)$, and a mean of 10.1 years $(S D=13.85)$ since amputation. Seven subjects were amputated due to a vascular cause, 10 from traumatic causes and four due to undisclosed causes, all with a K2 ambulation activity level. ${ }^{57}$ Ten subjects in the control group received the SSB socket design with a foam interface and supracondylar suspension, while the 11 participants in the experimental group received the TSB design, utilising a silicone liner and distal pin suspension with ICEX technique. All subjects were previously wearing prostheses with an SSB socket design with cuff suspension, standardised componentry and were walking without any gait aids at the time of recruitment. Baseline evaluations of gait analysis were conducted with the pre-study prosthesis and then repeated six weeks after the new TSB or SSB sockets were fitted.

Function and mobility was assessed in a gait laboratory, with gait registered via a VICON 370 3-dimensional motion analysis system with one Kistler force plate, using a standard set of reflective markers. Comfort and pain was assessed with use of the Socket Comfort Score, ${ }^{36}$ however standard deviation was not reported and these results could not be combined in meta-analysis.

Hachisuka et al., 199848a, 48b

This quasi-experimental before and after study sought to compare the advantages, disadvantages and clinical implications of the TSB and SSB socket designs for transtibial amputees. Data was collected from 32 participants, who were categorised as belonging to one of two groups: inpatients and outpatients. For the purposes of this review, the Hachisuka et al. study was divided into two parts: Hachisuka A relates to the 23 outpatient established amputee subjects ${ }^{48 a}$, and Hachisuka $B$ relates to the nine new amputees undergoing inpatient rehabilitation. $48 \mathrm{~b}$ Of the combined 32 subject sample, 27 were male and five female, with a mean age of 44.5 years $(S D=16.0)$ and mean months since amputation 10.9 (SD=13.8 months), with 21 amputations due to traumatic causes, seven due to vascular causes and four of which the cause was undisclosed.

Hachisuka A subjectively assessed satisfaction and preference for socket design with the 23 community-based outpatients who had their previous PTB KBM socket replaced 
with a TSB design. Participants' socket preference was identified via the use of a selfreported satisfaction survey.

Hachisuka B reports on nine inpatients that had not previously used a prosthesis and were given both the TSB and SSB designs, which were worn throughout the rehabilitation process a few days at a time, so a comparison during gait re-learning could be made. Patient socket preference was identified using the same self-report satisfaction survey as was used for the outpatient group.

Engsberg et al., 200650

This RCT study compared rectified and unrectified sockets for trans-tibial amputees. Authors were contacted for clarification on this terminology and confirmed that rectified referred to the situation whereby the positive plaster mould underwent manual modification to manufacture a specific weight bearing socket design (SSB). Whereas an unrectified socket was a positive mould taken via an alginate gel casting process, retaining the residual limb shape without modification, therefore creating a total surface bearing socket design (TSB).

A total of 43 subjects with a mean age of 47 years (SD=10 years), with amputations due to undisclosed causes, all independent ambulators without significant health problems, with mature residuum's and wearing a prosthesis continuously for a minimum of two years, were recruited. Subjects were randomly assigned to receive a prosthesis with TSB or SSB socket design, worn for a minimum of four weeks and then changed to the alternate design for the same period of time, for the purpose of acclimatisation. Both foam and gel interfaces were used in the socket designs, however when contacted, the authors could not say with confidence which type of interface was used with the TSB or SSB design. Therefore this study was used for TSB and SSB comparison and analysis, though not included in the sub-group analyses for interface and suspension modes. Outcomes including gait characteristics, socket preference, quality of life assessments and energy expenditure were measured after each socket was worn for the duration of a four-week period.

Function and mobility was assessed through use of a gait laboratory. Gait was assessed using a nine-metre walkway, with three-dimensional motion analysis system, six infrared cameras and one Kistler force plate. Six reflective markers were placed on 
anatomical landmarks of the pelvis, trunk and lower limbs. Quality of Life was assessed using the nine subscales of the PEQ. 35

Energy expenditure was calculated by investigating oxygen uptake, measured during a graded exercise test. Thirty-six subjects participated in this test because the sample was screened for fitness prior to participating in this exertive test.

Manucharian, 201153

This pseudo-RCT aimed to investigate whether differences exist in the comfort level of the hand casted SSB socket design and the more hands-off method of production of the TSB socket design. The study participants consisted of 36 subjects, the majority (30) with amputations due to vascular causes and the remaining six due to traumatic causes. The data produced by this study relates to the results of the Socket Comfort Score (SCS), a single-question self-report tool with 11-point numerical rating scale ranging from 0 to 10 , where 0 represents the most uncomfortable and 10 the most comfortable socket imaginable. ${ }^{36}$

Subjects were all previously wearing the SSB or TSB socket designs and were assigned to two groups: 21 to the SSB group and 15 to the TSB group, in which they were fitted with their new socket designs. The TSB design used the hydrocast technique, whilst the SSB socket was made using the traditional hand-cast technique. Both socket designs used a foam interface with knee sleeve suspension and distal expulsion valve. Comfort was measured with SCS upon delivery of the new prosthesis and after one month of use.

Klute et al., 201151

This RCT with cross-over design investigated the effect of two different suspension systems; vacuum assisted suction suspension (VASS) and pin suspension, after three weeks acclimatisation. The VASS was reported as being a TSB design and the pin system as an SSB design; the study therefore met the inclusion criteria for the systematic review.

Twenty subjects were recruited however 15 withdrew due to an inability to tolerate the vacuum TSB system, leaving five subjects enrolled in the study. The cause of amputation of four of these participants was trauma and a vascular cause in the remaining participant. The sample had a mean age of 56 years ( $S D=9$ years) and a 
mean of 13 years ( $S D=15$ years) since amputation, with all five subjects wearing the pin suspension SSB socket design prior to recruitment.

Function and mobility were assessed through use of an activity monitor worn throughout the day in participants' everyday life setting and recording step count, reported as steps per day. The activity monitor was worn for a consecutive two-week period, following three weeks of acclimatisation. Quality of Life was assessed using three subscales from the PEQ. ${ }^{35}$

Selles et al., $2005^{52}$

This prospective RCT compared the functional outcome of the TSB and SSB socket designs. Twenty-six adult unilateral trans-tibial amputees were recruited; 14 due to vascular causes, 11 because of traumatic causes and one of unknown cause. The sample had a mean age of 62.7 years ( $S D=14.5$ years) and all participants were experienced prosthesis users, having previously used the SSB socket design (PTB or KBM style) up until and including the study baseline.

Subjects were randomised to two groups: one group were provided with a newly manufactured TSB design socket with silicone gel liner and pin suspension with ICEX technique; the other group were fitted with a newly manufactured SSB style socket with silicone gel liner and pin suspension with hand-cast technique. Standardised manufacturing techniques were used and the same prosthetist occupied for socket fabrication and fitting. This was the only study included in this review that used the same type of suspension and interface for both groups, with socket design being the only true variation between the groups. Outcome measures including gait characteristics, presence and impact of pain or phantom pain, satisfaction and socket preference were assessed at baseline and after a period of three months acclimatisation with the new socket fitting.

Function and mobility were assessed through the use of a gait laboratory and community-based step activity monitor. Gait was assessed using a 15-metre straight track with three-dimensional motion analysis system and three infrared cameras; with sampling at $50 \mathrm{~Hz}$. Standard sets of reflective markers were placed on anatomical landmarks of the pelvis and lower limbs. An activity monitor was worn by each participant for two days in their everyday environment to monitor the frequency of activities of daily living, specifically the motions of sitting, standing, stair walking, and 
time spent in dynamic activities being percentage of 24 hours spent walking, stair walking, engaged in cyclic and in non-cyclic movements.

Quality of life was assessed using the PEQ 'satisfaction' subscale. ${ }^{35}$ Comfort and pain were also assessed using the PEQ. ${ }^{35}$

Astrom et al., 200454

This quasi-experimental before and after study compared the effect of TSB and SSB socket designs on measures of gait and socket comfort, for 29 trans-tibial amputees. A sub-group of seven subjects were confirmed to have only had the SSB design at study commencement and were then fitted with the gel interface TSB design during the study. The authors were approached for confirmation that all 29 subjects received the SSB design prior to receiving the TSB design. Confirmation of this was not received; therefore only the data available from the sub-group for which it was explicitly stated in the paper that the SSB socket design had been used prior to participation in the study.

Data was collected from this sub-group of four males and three females, with amputation cause being four non-vascular and three dysvascular, all of whom underwent gait analysis. Subjects were on average nine years (range: 1-39 years) postamputation, with a mean age of 46 years (range: 23-71 years of age). The socket design previously worn by the participants was the SSB hard outer socket with KBM suspension, with five subjects using a silicone gel interface and two using a foam interface. The TSB design intervention socket used a polyurethane gel liner with knee sleeve and distal expulsion valve to create a negative pressure suspension.

Walking speed was analysed in a gait laboratory setting at baseline with the SSB socket design, and again after two months acclimatisation with the TSB socket design. Gait was registered with the VICON 370 three-dimensional motion analysis system using one Kistler force plate, five infrared cameras, a data station and PC workstation. The sampling rate was $50 \mathrm{~Hz}$. Thirteen reflective markers were placed on anatomical landmarks of the pelvis and lower limbs. Comfortable walking speed and fast walking speed were recorded using a 10-metre walkway. All subjects wore the same foot and ankle componentry for both analyses with their own comfortable shoes and using the same prosthetist occupied to align the prostheses.

Yigiter et al., $2002^{55}$ 
This quasi-experimental before and after study compared the impact of the SSB and TSB socket designs on prosthetic rehabilitation. Data was collected from 20 subjects, with a mean age of 27.8 years ( $S D=7$ years, range $=15-37$ years), all with amputations of traumatic origin and presently being treated in a hospital-based rehabilitation setting, yet to receive their ambulatory prosthesis. This study was unique within the present review, as all subjects were new amputees undertaking primary rehabilitation. Subjects were fitted with both the TSB and SSB socket designs and provided with 10 days training in using both socket designs. Outcome measures were assessed after fitting and training with each socket design.

Function and mobility was assessed through analysis of gait and activities of daily living. Gait was registered through footprint analysis on a 12-metre walkway with measures recorded from the central seven metres.

Activities of daily living (ADL) were assessed via tasks of ascending and descending inclines and stairs, donning and doffing the prosthesis, picking up objects from the floor, crossing obstacles and sitting on and standing up from a chair.

Quality of Life was assessed through subjects' selection of which socket they would prefer to keep at the conclusion of rehabilitation.

Datta et al., 199656

This descriptive retrospective case series utilised a sample consisting of 54 subjects; 27 with amputations due to traumatic causes, 11 due to vascular causes, six congenital and 10 for which the cause was undisclosed. Participants had an average age of 48 years (range: $22-80$ years). Participants completed a postal questionnaire relating to their experience using the traditional SSB prosthetic socket design with foam interface and supracondylar suspension, compared to the recently provided ICEROSS TSB socket design with gel liner and pin lock suspension. A three page composite questionnaire was devised to gather information relating to personal experience with the new material, technique in regards to function, comfort and pain and ease of use. Due to the inconsistency of the instrument used to measure 'comfort and pain' compared to the other included studies, the data for this variable was not pooled in a meta-analysis, but presented in narrative form in the sub-group analysis for comfort and pain. There was no difference identified between socket designs $(p=0.68)$ in relation to the comfort of the prosthetic socket. 
The remaining elements of the questionnaire from which data was not extracted, covered client opinions and experiences on using walking aids indoors, outdoors and in bad weather, incidence of residuum breakdown and presence of pain, problems with sweating with interface liners, comfort of walking long distances and over rough terrain and climbing up and down stairs. The advantages and disadvantages of both the ICEROSS TSB and SSB systems were identified.

\subsection{Synthesised findings}

\section{Methods used in synthesis}

Although all the studies compared the TSB design with the SSB design, there were differences identified with respect to the interface and suspension methods used. To better understand how these different elements may influence the overall effect of the socket design, sub-group analyses were undertaken.

Studies that used a different measurement instrument for the same outcome were not pooled and the results of these are combined in a narrative summary. The descriptive case series data was also included in this review in a narrative form.

Data for all studies was extracted using the Joanna Briggs Institute (JBI) Meta-Analysis of Statistics Assessment and Review Instrument (MAStARI) and then transferred to the Cochrane Review Manager Version 5.2,47 to generate the forest plots, as this computer software allowed for further subgroup exploration in terms of the influence of different suspension modes and interface utilised within the intervention and comparator sockets. An alpha level of $p=0.05$ was used for all statistical tests. The fixed effects model Mantel-Haenzel was chosen initially, however, in the presence of statistically significant heterogeneity between the studies, a random effects model of Dersimonian \& Laird was employed to account for inter- and intra-study variation.

Synthesised results by health outcome domain

Function and mobility

Function and mobility was assessed in seven studies using 12 different measures. Physical performance was evaluated by 11 measures; with eight conducted in the laboratory setting, two undertaken in the community setting and one in both settings. One measure of ambulation was assessed using a self-report questionnaire. 
Physical performance measures included laboratory-based gait analyses to assess walking speed, step length, step width, temporal and spatial asymmetries and cadence. Physical performance measures conducted in rehabilitation facilities included weight bearing and balance. Community-based performance measures were wear time of prosthesis and steps per minute per day as measured through use of an activity monitor. Activities of daily living (ADLs) were recorded through facility-based performance and use of activity monitors in the community setting for use with everyday living activities. Subscales of the Prosthesis Evaluation Questionnaire (PEQ) were used to report on function, mobility and ambulation. ${ }^{35}$

Outcomes, instruments used and the studies assessing these are reported below.

\subsubsection{Laboratory-based physical performance measures}

Gait analysis

Instrumented gait analysis use in prosthetic evaluation provides better insights and knowledge of the different adaptive mechanisms of the body when walking with a prosthesis compared to visual observation. ${ }^{58}$ Most instrumented gait studies are conducted in a gait laboratory, with a measured walkway and high resolution cameras which take video footage detecting reflective markers placed specifically on body segments and joint axes. Force plates are often embedded into the walkway to allow for ground reaction force capture and analysis. Gait analysis is used in clinical settings to assist diagnosis, to allow before-and-after comparisons with specific interventions, or to document changes over time, which can influence treatment, on the basis of the clinician's interpretations of gait characteristics.

\subsubsection{Walking speed}

Walking speed is assessed as the distance travelled over time. In rehabilitation, walking speed is used as a measure of ambulatory confidence and ability. However, depending on population characteristics, walking speed may be influenced. Older populations, individuals with vascular aetiology, or amputees with co-morbidities would be expected to exhibit a decreased walking speed due to the impact of these variables on their general health and functionality.

Five studies measured walking speed within a gait laboratory environment and calculated walking speed in metres per second $(\mathrm{m} / \mathrm{sec}) .{ }^{24,50,52,54,55}$ Four studies used a 
gait laboratory with motion capture via high resolution cameras, using reflective standard marker sets, converting data to three-dimensional images for analysis of gait characteristics. ${ }^{24,50,52,54}$ However, the length of the walkway differed between these studies: Engsberg used a nine metre walkway, capturing data in the middle two metres; 50 Astrom used a 10-metre walkway; ${ }^{54}$ and Selles used a 15-metre walkway. ${ }^{52}$ Datta did not disclose the length of the walkway, however it is assumed from the description of the gait laboratory equipment used, that a minimum distance of nine metres would have been used for the walkway. ${ }^{24}$ One study, Yigiter et al., measured walking speed via footprint analysis using a 12-metre walkway with data captured from the central seven metres. ${ }^{55}$ Reason for amputation, participant numbers and study results are detailed in Table 3. Results are combined statistically and the meta-analysis is presented in Figure 2.

Table 3: Studies included in the meta-analyses (Figures 2, 3 and 4) reporting on walking speed measured in metres per second.

\begin{tabular}{|c|c|c|c|c|}
\hline Study ID & Study design & Participants & $\begin{array}{l}\text { Intervention/ } \\
\text { comparator }\end{array}$ & $\begin{array}{l}\text { Walking speed } \\
\text { mean (SD) } \mathrm{m} / \mathrm{sec}\end{array}$ \\
\hline $\begin{array}{l}\text { Astrom } \\
2004^{54}\end{array}$ & $\begin{array}{l}\text { Quasi-experimental } \\
\text { before and after }\end{array}$ & $\begin{array}{l}\mathrm{n}=7 \\
\text { Aetiology: V/T/O (3/0/4) }\end{array}$ & $\begin{array}{l}\text { TSB: GI, SI-vS } \\
\text { SSB: FI/GI, SC-S }\end{array}$ & $\begin{array}{l}\text { TSB } 1.16(0.23) \\
\text { SSB } 1.05(0.25)\end{array}$ \\
\hline $\begin{array}{l}\text { Datta } \\
2004^{24}\end{array}$ & Experimental RCT & $\begin{array}{l}\mathrm{n}=21 \\
\text { Aetiology: V/T/O (7/10/4) }\end{array}$ & $\begin{array}{l}\text { TSB: GI, PS } \\
\text { SSB: FI, SC-S }\end{array}$ & $\begin{array}{l}\text { TSB } 1.08(0.26) \\
\text { SSB } 1.01(0.24)\end{array}$ \\
\hline $\begin{array}{l}\text { Engsberg } \\
2006^{50}\end{array}$ & Experimental RCT & $\begin{array}{l}\mathrm{n}=43 \\
\text { Aetiology: V/T/O (unknown) }\end{array}$ & $\begin{array}{l}\text { TSB } \\
\text { SSB }\end{array}$ & $\begin{array}{l}\text { TSB } 1.25(0.22) \\
\text { SSB } 1.25(0.22)\end{array}$ \\
\hline $\begin{array}{l}\text { Selles } \\
2005^{52}\end{array}$ & Experimental RCT & $\begin{array}{l}\mathrm{n}=26 \\
\text { Aetiology: V/T/O (14/11/1) }\end{array}$ & $\begin{array}{l}\text { TSB: GI, PS } \\
\text { SSB: GI, PS }\end{array}$ & $\begin{array}{l}\text { TSB } 0.92(0.28) \\
\text { SSB } 0.92(0.31)\end{array}$ \\
\hline $\begin{array}{l}\text { Yigiter } \\
2002^{55}\end{array}$ & $\begin{array}{l}\text { Quasi-experimental } \\
\text { before and after }\end{array}$ & $\begin{array}{l}\mathrm{n}=20 \\
\text { Aetiology: V/T/O (0/20/0) }\end{array}$ & $\begin{array}{l}\text { TSB: GI, PS } \\
\text { SSB: FI, SC-S }\end{array}$ & $\begin{array}{l}\text { TSB } 0.74(0.11) \\
\text { SSB } 0.66(0.11)\end{array}$ \\
\hline
\end{tabular}

Abbreviations: TSB: Total surface bearing; SSB: Specific surface bearing; GI: Gel Interface; FI: Foam Interface; PS: Pin Suspension; SI-vS: Sleeve and valve suspension; SC-S: Supracondylar suspension; Aetiology =V: Vascular, T: Traumatic; O: Other; Yrs: Years; SD: Standard deviation 
Walking speed was analysed for a total of 187 participants across five studies. The weighted mean difference (WMD) was calculated for the mean walking speed of both socket designs. Three of the five studies reported faster walking speeds with the TSB socket groups. Two studies reported no difference in walking speed. The meta-analysis shows an overall effect size of $(\mathrm{WMD}=0.05 ; \mathrm{Cl}=0.00,0.10 ; p=0.04)$, using a random effects model. Although no statistical heterogeneity was detected between the studies (Chi square $=2.28, p=0.68,1^{2}=0 \%$ ), given the small number of studies, heterogeneity cannot be excluded.

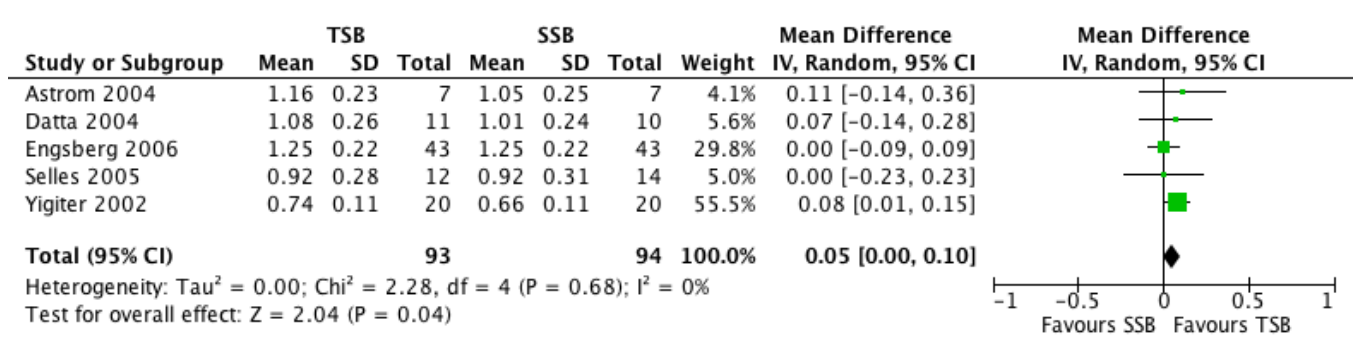

Figure 2: Gait analysis (Walking speed measured in metres/second)

The combined data shows an increase in walking speed with the TSB socket; however this was not a statistically significant difference compared to walking speed with the SSB socket design. Clinically, the increase of $5 \mathrm{~cm}$ per second is not considered significant over such short distances; however this increase may be of clinical significance over longer distances.

A potentially confounding factor in this analysis was the use of different interfaces within the socket groups. Therefore a subgroup analysis was conducted for the three studies that used the gel interface within the TSB design and the foam interface within the SSB design. $24,54,55$ Data was pooled for these three studies and is presented in Figure 3.

Walking speed was combined for 75 participants across the three studies using gel interfaces in the treatment (TSB) group and foam interfaces in the comparator (SSB) groups. All three studies reported increased walking speed with the TSB socket and when combined statistically this was a significant difference. The meta-analysis of data showed an overall effect size of $(\mathrm{WMD}=0.08 ; \mathrm{Cl}=0.02,0.14 ; \mathrm{p}=0.01)$ using a random effects model. There was no statistical heterogeneity found to exist between the studies (Chi square $=0.06, p=0.97,\left.\right|^{2}=0 \%$ ). 


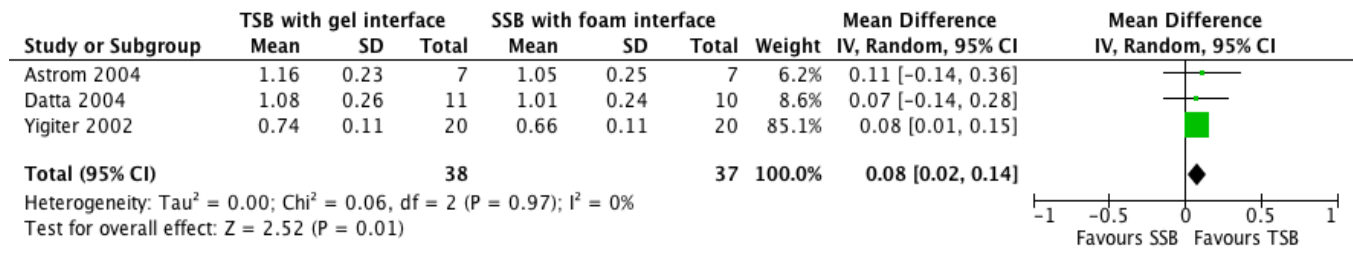

Figure 3: Gait analysis (Walking speed measured in metres/second for socket designs with gel interfaces for TSB group and foam interface for SSB group)

The significance of these results, although a small difference $(8 \mathrm{~cm})$ measured in a controlled laboratory environment, may be considered clinically significant as it translates to an improvement in mobility in the home environment or community setting when longer distances are required for general daily activities.

A further factor that may have affected the size and direction of the effect measure may be differences in the type of suspension used with each socket in the studies. This was examined by undertaking a subgroup analysis, shown below in Figure 4. Distal pin lock suspension was used with the TSB design and gel interface and supracondylar suspension was used with the SSB design with foam interface.

There were 61 participants who had their gait analysed to measure walking speed across two studies, using the interfaces and suspension method described above. ${ }^{24,55}$ The meta-analysis shows an effect size of $(\mathrm{WMD}=0.08 ; \mathrm{Cl}=0.01,0.14 ; \mathrm{p}=0.02)$ using a random effects model. There was no statistical heterogeneity found between the studies (Chi square $=0.01, p=0.93,1^{2}=0 \%$ ).

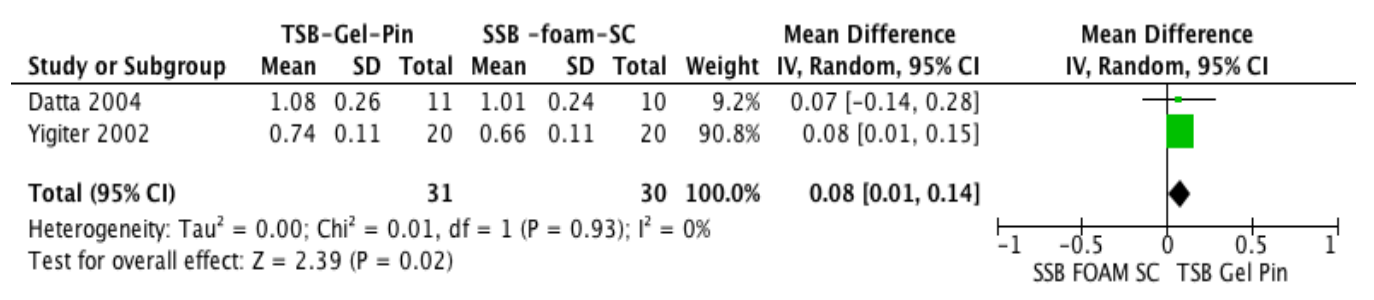

Figure 4: Gait analysis (Walking speed measured in metres/second for socket designs with gel interfaces and distal suspension for TSB group and foam interface with proximal suspension for the SSB group)

In summary, an increase in walking speed was found with the TSB socket design compared to the SSB design. Subgroup analysis that explored variation in the interface and suspension method indicated a statistically significant improvement in walking speed with the TSB design using a gel interface combined with distal pin suspension. 
The sub-group analyses included participants with a lower mean age and a higher proportion of amputations due to non-vascular reasons. Regardless of these notations, it is the intra-subject comparison of change in metres/second that is being investigated and when the younger traumatic amputee participants record an increased walking speed at baseline, there is evidence of improvement in walking speed when using the TSB design. In a clinical setting, improved walking speed is considered to be a marker of improved balance, mobility and an indicator of comfort.

Clinically, a small increase may not be of great significance over short distances, however over longer distances where endurance is required, an increase in metres per second could translate into a useful functional achievement. The improvement may be attributed to the gel interface liners that provide an improved connection between the residuum and the socket; and distal pin suspension that acts to reduce the pistoning of the residuum within the socket, rather than the difference in socket design alone.

\subsubsection{Stride length}

Stride length is the distance between two successive placements of the same foot, consisting of two step-lengths, left and right, measured in centimetres and assessed under laboratory conditions. Five studies reported on stride length: four within a laboratory setting as described for 'walking speed',24,50,52,55 and one study within the community setting utilising an activity monitor. ${ }^{23}$ Population characteristics and outcomes are presented in Table 4. 
Table 4: Studies reporting stride length in metres.

\begin{tabular}{|c|c|c|c|c|}
\hline Study ID & Study design & Participants & $\begin{array}{l}\text { Intervention/ } \\
\text { comparator }\end{array}$ & $\begin{array}{l}\text { Stride length } \\
\text { mean (SD) }\end{array}$ \\
\hline $\begin{array}{l}\text { Datta } \\
2004^{24}\end{array}$ & Experimental RCT & $\begin{array}{l}\mathrm{n}=21 \\
\text { Aetiology: V/T/O (7/10/4) }\end{array}$ & $\begin{array}{l}\text { TSB: GI, PS } \\
\text { SSB: FI, SC-S }\end{array}$ & $\begin{array}{l}\text { TSB } 1.25(0.24) \\
\text { SSB } 1.21(0.27)\end{array}$ \\
\hline $\begin{array}{l}\text { Engsberg } \\
2006^{50}\end{array}$ & Experimental RCT & $\begin{array}{l}n=43 \\
\text { Aetiology: V/T/O (unknown) }\end{array}$ & $\begin{array}{l}\text { TSB } \\
\text { SSB }\end{array}$ & $\begin{array}{l}\text { TSB } 1.44(0.18) \\
\text { SSB } 1.43(0.17)\end{array}$ \\
\hline $\begin{array}{l}\text { Selles } \\
2005^{52}\end{array}$ & Experimental RCT & $\begin{array}{l}\mathrm{n}=26 \\
\text { Aetiology: V/T/O (14/11/1) }\end{array}$ & $\begin{array}{l}\text { TSB: GI, PS } \\
\text { SSB: GI, PS }\end{array}$ & $\begin{array}{l}\text { TSB } 1.15(0.22) \\
\text { SSB } 1.12(0.30)\end{array}$ \\
\hline $\begin{array}{l}\text { Yigiter } \\
2002^{55}\end{array}$ & $\begin{array}{l}\text { Quasi-experimental } \\
\text { before and after }\end{array}$ & $\begin{array}{l}\mathrm{n}=20 \\
\text { Aetiology: V/T/O (0/20/0) }\end{array}$ & $\begin{array}{l}\text { TSB: GI, PS } \\
\text { SSB: FI, SC-S }\end{array}$ & $\begin{array}{l}\text { TSB } 1.12(0.10) \\
\text { SSB } 1.09(0.14)\end{array}$ \\
\hline $\begin{array}{l}\text { Coleman } \\
2004^{23}\end{array}$ & Experimental RCT & $\begin{array}{l}\mathrm{N}=13 \\
\text { Aetiology: } \mathrm{V} / \mathrm{T} / \mathrm{O}(0 / 13 / 0)\end{array}$ & $\begin{array}{l}\text { TSB: GI, PS } \\
\text { SSB: FI, SC-S }\end{array}$ & $\begin{array}{l}\text { TSB } 1.38(0.04) \\
\text { SSB } 1.38(0.04)\end{array}$ \\
\hline
\end{tabular}

Abbreviation: TSB: Total surface bearing; SSB: Specific surface bearing; GI: Gel Interface; FI: Foam Interface; PS: Pin Suspension; SC-S: Supracondylar suspension; Aetiology=V: Vascular, T: Traumatic, O: Other; Yrs: Years; SD: Standard deviation

Stride length was analysed for a total of 173 participants across four studies. The metaanalysis (Figure 5) shows a weak effect size of (WMD=0.02; Cl=-.03, .07) using a random effects model. No statistical difference $(p=0.40)$ was observed in stride length comparing the TSB and SSB socket designs. There was no statistical heterogeneity found between the studies (Chi square $=0.18, p=0.98,{ }^{2}=0 \%$ ).

\begin{tabular}{|c|c|c|c|c|c|c|c|c|c|}
\hline Study or Subgroup & \multicolumn{3}{|c|}{ TSB } & \multicolumn{3}{|c|}{ SSB } & \multicolumn{2}{|r|}{ Mean Difference } & $\begin{array}{l}\text { Mean Difference } \\
\text { IV, Random, } 95 \% \mathrm{CI}\end{array}$ \\
\hline Datta 2004 & 1.25 & 0.24 & 11 & 1.21 & 0.27 & 10 & $5.1 \%$ & $0.04[-0.18,0.26]$ & \\
\hline Engsberg 2006 & 1.44 & 0.18 & 43 & 1.43 & 0.17 & 43 & $45.2 \%$ & $0.01[-0.06,0.08]$ & \\
\hline Selles 2005 & 1.15 & 0.22 & 12 & 1.12 & 0.3 & 14 & $6.2 \%$ & $0.03[-0.17,0.23]$ & \\
\hline Yigiter 2002 & 1.12 & 0.1 & 20 & 1.09 & 0.14 & 20 & $43.5 \%$ & $0.03[-0.05,0.11]$ & \\
\hline Total $(95 \% \mathrm{CI})$ & & & 86 & & & 87 & $100.0 \%$ & $0.02[-0.03,0.07]$ & \\
\hline \multicolumn{9}{|c|}{$\begin{array}{l}\text { Heterogeneity: } \mathrm{Tau}^{2}=0.00 ; \mathrm{Chi}^{2}=0.18, \mathrm{df}=3(\mathrm{P}=0.98) ; \mathrm{I}^{2}=0 \% \\
\text { Test for overall effect: } \mathrm{Z}=0.85(\mathrm{P}=0.40)\end{array}$} & $\begin{array}{ccc}-0.5 & 0.25 & 0.5 \\
\text { Favours SSB] } & \text { Favours T }\end{array}$ \\
\hline
\end{tabular}

Figure 5: Gait analysis (Stride length measured in metres comparing the TSB and SSB socket designs)

As was the case with the measurement of walking speed, the type of interface and/or suspension method may have confounded the estimate of effect of stride length. 
Therefore, the three studies that used the same interfaces and suspension methods in their TSB sockets, and the same interface and suspension modes for their SSB sockets were examined and subgroup analyses reported.

Gait was analysed for 61 participants in order to measure stride length using a gel interface and distal pin suspension within the TSB group compared with the SSB group utilising a foam interface and proximal suspension method. The meta-analysis (Figure 6) shows a small effect size of $(\mathrm{WMD}=0.03 ; \mathrm{Cl}=-0.04,0.10)$ using a random effects model, with no statistical difference $(p=0.39)$ observed. No statistical heterogeneity was detected between the studies (Chi square $=0.01, p=0.93,12=0 \%$ ), however only two studies were included in this meta-analysis. ${ }^{24,55}$

\begin{tabular}{|c|c|c|c|c|c|c|c|c|c|}
\hline \multirow[b]{2}{*}{ Study or Subgroup } & \multicolumn{3}{|c|}{ TSB-Gel-Pin } & \multicolumn{3}{|c|}{ SSB -foam-SC } & \multirow[b]{2}{*}{ Weight } & \multirow{2}{*}{$\begin{array}{l}\text { Mean Difference } \\
\text { IV, Random, 95\% CI }\end{array}$} & \multirow{2}{*}{$\begin{array}{c}\text { Mean Difference } \\
\text { IV, Random, } 95 \% \mathrm{CI}\end{array}$} \\
\hline & Mean & SD & Total & Mean & SD & Total & & & \\
\hline Datta 2004 & 1.25 & 0.24 & 11 & 1.21 & 0.27 & 10 & $10.6 \%$ & $0.04[-0.18,0.26]$ & \\
\hline Yigiter 2002 & 1.12 & 0.1 & 20 & 1.09 & 0.14 & 20 & $89.4 \%$ & $0.03[-0.05,0.11]$ & \\
\hline Total $(95 \% \mathrm{CI})$ & & & 31 & & & 30 & $100.0 \%$ & $0.03[-0.04,0.10]$ & \\
\hline \multicolumn{9}{|c|}{$\begin{array}{l}\text { Heterogeneity: } \text { Tau }^{2}=0.00 ; \mathrm{Chi}^{2}=0.01, \mathrm{df}=1(\mathrm{P}=0.93) ; \mathrm{I}^{2}=0 \% \\
\text { Test for overall effect: } \mathrm{Z}=0.85(\mathrm{P}=0.39)\end{array}$} & 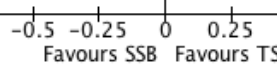 \\
\hline
\end{tabular}

Figure 6: Gait analysis (Stride length measured in metres comparing the TSB with gel interfaces and distal suspension and the SSB socket design with foam interface with proximal suspension)

Coleman et al. ${ }^{23}$ reported on stride length as determined by a Step Activity Monitor worn by participants in their everyday home or community setting over a two week period and used the same interface and suspension modes as Yigiter ${ }^{55}$ and Datta. ${ }^{24}$ However, these results were not included in the meta-analysis (Figure 6) as the data was recorded in the community-based setting and therefore under less controlled conditions as a laboratory. As detailed in Table 4, no difference was observed in the stride length of participants wearing the two socket designs.

In summary, there was no difference in stride length between the two socket designs; regardless of interface or suspension system utilised and irrespective of analysis setting, laboratory-based or in the home environment. Stride length is likely related to individual walking style, leg length and physiological features rather than mechanical elements of the prosthesis. 


\subsubsection{Cadence}

Cadence is the number of steps per unit of time and is usually calculated in steps per minute. It is related to both walking speed and step length. Three studies assessed cadence in a laboratory-based environment. Two studies used a motion capture system for analysis, $, 4,50$ and one used a footprint analysis. ${ }^{5}$ These methods are similar enough in their data capture of temporal measures for them to be appropriately combined in a meta-analysis. Participant, socket design and outcomes are described in Table 5, with results combined in meta-analysis and shown in Figure 7.

Table 5: Studies included in the meta-analysis that assessed cadence measured as steps per minute.

\begin{tabular}{|l|l|l|l|l|}
\hline Study ID & Study design & Participants & $\begin{array}{l}\text { Intervention/ } \\
\text { comparator }\end{array}$ & $\begin{array}{l}\text { Cadence steps/min } \\
\text { mean (SD) }\end{array}$ \\
\hline $\begin{array}{l}\text { Datta } \\
\mathbf{2 0 0 4} \mathbf{2 4}^{24}\end{array}$ & Experimental RCT & $\mathrm{n}=21$ & $\begin{array}{l}\text { TSB: GI, PS } \\
\text { SSB: FI, SC-S }\end{array}$ & TSB 102.14 (7.32) \\
\hline $\begin{array}{l}\text { Engsberg } \\
\mathbf{2 0 0 6}\end{array}$ & Expetiology: V/T/O (7/10/4) & SS9.7 (8.90) \\
\hline $\begin{array}{l}\text { Yigiter } \\
\mathbf{2 0 0 2 5}\end{array}$ & Quasi-experimental & $\mathrm{n}=20$ & TSB & TSB 105 (11) \\
\hline
\end{tabular}

Abbreviation: TSB: Total surface bearing; SSB: Specific surface bearing; Gl: Gel Interface; Fl: Foam Interface; PS: Pin Suspension; SC-S: Supracondylar suspension; Aetiology=V: Vascular, T: Traumatic, O: Other; Yrs: Years; SD: Standard deviation

Combining data from the three studies, there were 147 participants with gait analysed to measure cadence. The meta-analysis showed a large effect size of $(\mathrm{WMD}=3.02$; $\mathrm{Cl}=-1.25,7.30 ; \mathrm{p}=0.17$ ) using a random effects model; however there was no statistically significant difference between the cadence of the TSB and SSB groups as the confidence interval crosses the 'line of no effect'. There was moderate statistical heterogeneity found between the studies (Chi square=3.68, $p=0.16,1^{2}=46$ ). 


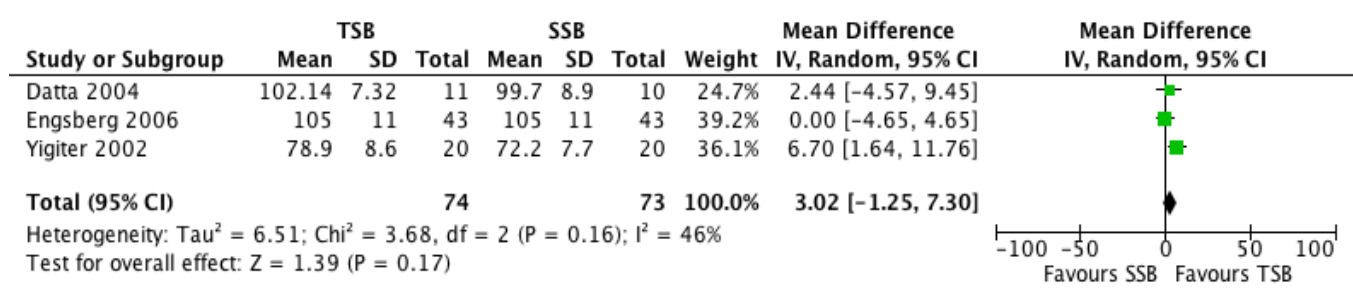

Figure 7: Gait analysis (Cadence measured by steps per minute comparing TSB socket design with SSB socket design irrespective of interfaces and suspension methods utilised)

As with walking speed and stride length, the type of interface and/or suspension mode may confound the estimate of the effect size for cadence. Therefore, a sub-group analysis was conducted in order to ascertain whether the results differed when controlling for these potential confounds. The two studies included in this sub-group analysis both utilised the gel interface with distal pin suspension in the TSB design and the foam interface with supracondylar suspension in the SSB socket design, ${ }^{24,55}$ whereas the study by Engsberg was excluded from this analysis as the primary author could not confirm which specific interface and suspension method was used. ${ }^{50}$ The results are shown below in meta-analysis Figure 8 .

There were 61 participants in the two studies measuring cadence in socket designs controlling for interface and suspension technique. The meta-analysis of data shows a large effect size of (WMD=5.24; $\mathrm{Cl}=1.14,9.34)$ using a random effects model. There was no statistical heterogeneity found between the studies (Chi square $=0.93, p=0.33$, $\left.{ }^{2}=0 \%\right)$. When examining only the same studies with the same interface and suspension methods, a statistically significant increase $(p=0.01)$ in cadence was seen for the TSB socket design. Clinically this may not be significant over short distances, though if endurance cadence was maintained this could be meaningful to the person with amputation walking longer distances. 


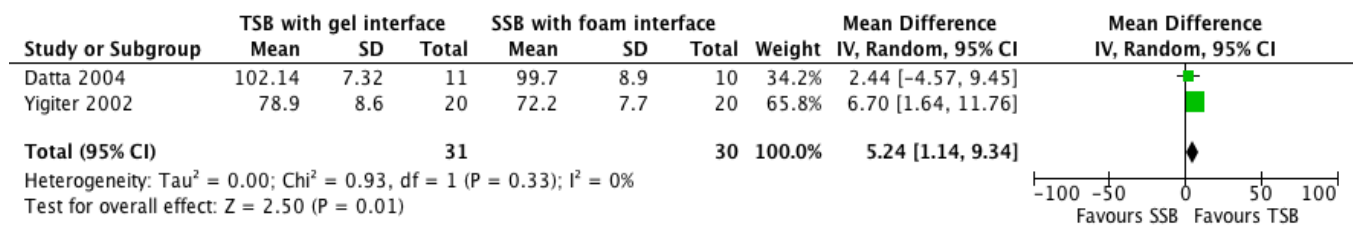

Figure 8: Gait analysis (Cadence measured by steps per minute comparing the TSB with gel interface and distal pin suspension and SSB socket design with foam interface and proximal suspension method)

In summary, there was no statistically significant difference in cadence, except when considering the effect of suspension methods and interfaces between the two designs. Although participants with the TSB socket design had on average more steps per minute, this improvement was not statistically significant. Improved cadence is likely attributable to the feature of the gel interface with distal pin suspension providing an intimate fit between residuum and socket, and suspension providing less pistoning within the socket compared to the foam and supra-condylar interface and suspension.

Asymmetries in gait: spatial and temporal

Persons with amputation typically exhibit asymmetrical or antalgic gait compared to 'healthy' persons (without amputation or lower-limb pathology). ${ }^{46}$ The soft tissues of the residuum are not well-suited for load bearing, therefore the intact (non-amputated) limb usually has increased stance time during the gait cycle, coupled with shortened swing time, to minimise the amount of time during which weight bearing occurs through the amputated limb. ${ }^{47}$ This contributes to an increase in the forces bared by the intact limb, possibly contributing to joint pain and degeneration. One aim of amputee prosthetic rehabilitation is to provide a comfortable prosthetic fit with good weight distribution characteristics and the goal of achieving symmetrical stance and swing phases to prevent undue joint loading of the intact limb.

\subsubsection{Temporal or step asymmetry}

Temporal or step asymmetry is the difference in the left step versus the right step in measured length. In healthy subjects symmetrical gait is expected, with equal length steps. It is important to compare intra-subject results in this review, to determine whether socket design has any effect on gait symmetry.

Two studies examined step asymmetry measured in a laboratory environment, where asymmetry was analysed by determining a Symmetry Index (SI). ${ }^{52,55}$ Selles et al. 
conceptualised $\mathrm{SI}$ as prosthetic step length divided by intact step length. ${ }^{52}$ If the resulting number was larger than one, the inverse $(1 / x)$ was calculated. This method results in the production of a number between zero and one, with one representing perfect symmetry. Yigiter et al. reported prosthetic and intact side step lengths for both the TSB and SSB designs, ${ }^{55}$ from which SI was calculated with the same method used by Selles, therefore allowing data from both studies to be combined in meta-analysis (Figure 9). ${ }^{52}$

Datta et al. also measured step asymmetry, although used a different calculation method to determine SI and the results therefore were not compatible for inclusion in the meta-analysis. ${ }^{24}$ The results from this study are presented in narrative form.

Outcomes from the three studies are summarised in Table 6.

Table 6: Studies assessing step asymmetry reported as a symmetry index.

\begin{tabular}{|l|l|l|l|l|}
\hline Study ID & Study design & Participants & $\begin{array}{l}\text { Intervention/ } \\
\text { comparator }\end{array}$ & $\begin{array}{l}\text { Step asymmetry } \\
\text { mean (SD) }\end{array}$ \\
\hline Selles & Experimental RCT & $\mathrm{n}=26$ & TSB: GI, PS & TSB $0.98(0.09)$ \\
$\mathbf{2 0 0 5 ^ { 5 2 }}$ & Aetiology: V/T/O (14/11/1) & SSB: GI, PS & SSB $0.92(0.09)$ \\
\hline $\begin{array}{l}\text { Yigiter } \\
\mathbf{2 0 0 2 5}\end{array}$ & Quasi-experimental & $\mathrm{n}=20$ & $\begin{array}{l}\text { TSB: GI, PS } \\
\text { SSB: FI, SC-S }\end{array}$ & TSB $0.98(0.98)$ \\
\hline $\begin{array}{l}\text { Datta } \\
\mathbf{2 0 0 4} \mathbf{2 4}^{24}\end{array}$ & Expere and after & Aetiology: V/T/O (0/20/0) & SSB (0.97) \\
\hline
\end{tabular}

Abbreviation: TSB: Total surface bearing; SSB: Specific surface bearing; Gl: Gel interface; FI: Foam interface; PS: Pin suspension; SC-S: Supracondylar suspension; Aetiology=V: Vascular, T: Traumatic, O: Other; Yrs: Years; SD: Standard deviation

There were 66 participants across the two studies with gait analysed to measure step asymmetry. ${ }^{22,23}$ When statistically combined using a random effects model, results revealed the TSB socket group had a slightly more symmetrical gait in terms of step asymmetry with a small effect size $(\mathrm{WMD}=0.06 ; \mathrm{Cl}=-0.01,0.13)$, however this difference is not statistically significant $(p=0.09)$. There was no statistically significant heterogeneity found between the studies (Chi square $=0.01, p=0.92,12=0 \%$ ). 


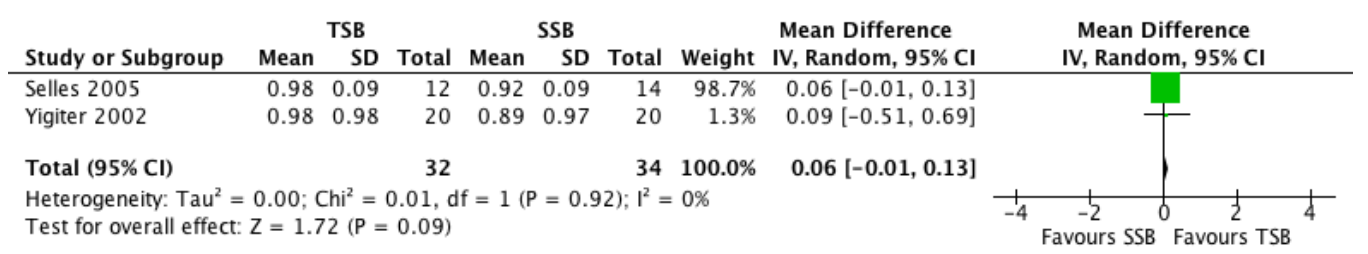

Figure 9: Gait analysis (Step asymmetry assessed with a symmetry index comparing the TSB with the SSB socket design)

Datta et al. ${ }^{24}$ reported on step asymmetry in their RCT to provide an overview of gait quality. The authors used the Herzog formula to calculate the SI. ${ }^{48}$ Mann-Whitney U tests were used in statistical evaluations with a significance level of .05 .

Mean (SD) symmetry index (SI) was reported for both groups. The SI for the SSB group was $7.95(S D=4.25)$ at baseline and $4.50(S D=4.35)$ at six weeks follow-up. SI data for the TSB group showed baseline data of 7.01 ( $S D=5.27$ ) with pre-study SSB socket compared to 7.28 (SD =0.94) with the new TSB socket. The authors found that there was no significant difference in walking symmetry between the socket designs.

In summary, considering the results from the meta-analysis (Figure 9) and results from Datta et al, no overall difference was observed in step asymmetry when wearing the SSB compared to the TSB socket design. ${ }^{24}$

\subsubsection{Spatial or stance asymmetry}

Stance asymmetry measures the amount of time spent on the prosthetic limb compared to the intact limb during a gait cycle. Two studies reported stance asymmetry measured in a laboratory environment, analysed by determining a Symmetry Index (SI).50,52 Selles et al. calculated SI in the same way as step asymmetry, with prosthetic stance-phase duration divided by intact stance-phase duration and if the resulting number was larger than 1 , the inverse $(1 / x)$ was calculated. ${ }^{52}$ Through this method a number between 0 and 1 is created, with 1 representing perfect symmetry. Engsberg et al., calculate stance $\mathrm{SI}$ in the same way, however reporting the result as a percentage. With $\mathrm{SI}$ calculated by like method, results were combined in meta-analysis shown in Figure 10.50

Datta et al., ${ }^{24}$ also measured stance asymmetry, however they used a different calculation method to determine SI and their results therefore could not be pooled 
within the meta-analysis. ${ }^{24}$ Results are included in narrative form. The three studies key design features and results are summarised in Table 7.

Table 7: Studies included assessing stance asymmetry reported as a symmetry index.

\begin{tabular}{|c|c|c|c|c|}
\hline Study ID & Study design & Participants & $\begin{array}{l}\text { Intervention/ } \\
\text { comparator }\end{array}$ & $\begin{array}{l}\text { Stance asymmetry } \\
\text { mean (SD) }\end{array}$ \\
\hline $\begin{array}{l}\text { Engsberg } \\
2006^{50}\end{array}$ & $\begin{array}{l}\text { Experimental } \\
\mathrm{RCT}\end{array}$ & $\begin{array}{l}n=43 \\
\text { Aetiology: V/T/O (unknown) }\end{array}$ & $\begin{array}{l}\text { TSB } \\
\text { SSB }\end{array}$ & $\begin{array}{l}\text { TSB } 0.97(0.06) \\
\text { SSB } 0.97(0.05)\end{array}$ \\
\hline $\begin{array}{l}\text { Selles } \\
2005^{52}\end{array}$ & $\begin{array}{l}\text { Experimental } \\
\text { RCT }\end{array}$ & $\begin{array}{l}\mathrm{n}=26 \\
\text { Aetiology: V/T/O (14/11/1) }\end{array}$ & $\begin{array}{l}\text { TSB: GI, PS } \\
\text { SSB: GI, PS }\end{array}$ & $\begin{array}{l}\text { TSB } 0.94(0.07) \\
\text { SSB } 0.94(0.05)\end{array}$ \\
\hline $\begin{array}{l}\text { Datta } \\
2004^{24}\end{array}$ & $\begin{array}{l}\text { Experimental } \\
\mathrm{RCT}\end{array}$ & $\begin{array}{l}\mathrm{n}=21 \\
\text { Aetiology: V/T/O (7/10/4) }\end{array}$ & $\begin{array}{l}\text { TSB: GI, PS } \\
\text { SSB: FI, SC-S }\end{array}$ & $\begin{array}{l}\text { TSB -13.45 (13.51) } \\
\text { SSB -10.93 (13.34) }\end{array}$ \\
\hline
\end{tabular}

Abbreviation: TSB: Total surface bearing; SSB: Specific surface bearing; Gl: Gel interface; FI: Foam interface; PS: Pin suspension; SC-S: Supracondylar suspension; Aetiology= V: Vascular, $T$ : Traumatic, O: Other; Yrs: Years; SD: Standard deviation

When the two studies were combined in meta-analysis (Figure 10), there were 112 participants with gait analysed to measure stance asymmetry. The meta-analysis shows an effect size of $(\mathrm{WMD}=0.00 ; \mathrm{Cl}=-0.02,0.02 ; \mathrm{p}=1.00)$ using a random effects model. There was no statistical heterogeneity found between the studies (Chi square $=0.00$, $p=1.00, \quad 2=0 \%)$. No statistically significant difference was observed in stance asymmetry between the groups comparing the TSB and SSB socket designs, as measured when calculating the symmetry index from the gait analysis.

\begin{tabular}{|c|c|c|c|c|c|c|c|c|c|}
\hline \multirow[b]{2}{*}{ Study or Subgroup } & \multicolumn{3}{|c|}{ TSB } & \multicolumn{3}{|c|}{ SSB } & \multicolumn{2}{|r|}{ Mean Difference } & \multirow{2}{*}{$\begin{array}{c}\text { Mean Difference } \\
\text { IV, Random, } 95 \% \mathrm{CI}\end{array}$} \\
\hline & Mean & SD & Total & Mean & SD & Total & Weight & IV, Random, 95\% CI & \\
\hline Engsberg 2006 & 0.97 & 0.06 & 43 & 0.97 & 0.05 & 43 & $80.5 \%$ & $0.00[-0.02,0.02]$ & \\
\hline Selles 2005 & 0.94 & 0.07 & 12 & 0.94 & 0.05 & 14 & $19.5 \%$ & $0.00[-0.05$ & \\
\hline Total $(95 \% \mathrm{Cl})$ & & & 55 & & & 57 & $100.0 \%$ & $0.00[-0.02,0.02]$ & \\
\hline \multicolumn{9}{|c|}{$\begin{array}{l}\text { Heterogeneity: } \text { Tau }^{2}=0.00 ; \mathrm{Chi}^{2}=0.00, \mathrm{df}=1(P=1.00) ; I^{2}=0 \% \\
\text { Test for overall effect: } Z=0.00(P=1.00)\end{array}$} & $\begin{array}{lccc}-1 & -0.5 & 0 & 0.5 \\
& \text { Favours SSB } & \text { Favours T }\end{array}$ \\
\hline
\end{tabular}

Figure 10: Gait analysis (Stance asymmetry assessed by a symmetry index calculation comparing the TSB socket design with the SSB socket design)

Datta et al., reported on stance asymmetry in their RCT with gait analysis conducted as described above for Step Asymmetry. ${ }^{24}$ The reported data was the calculated SI for 
both groups. The SSB group's SI was reported as $-9.51(S D=12.03)$ at baseline and $10.93(S D=13.34)$ at six weeks follow-up. SI data for the TSB group was -6.06 $(S D=8.96)$ at baseline with the previous SSB socket, compared with $-13.45(S D=13.51)$ with the new TSB socket. The authors reported no significant difference in stance asymmetry between the socket designs. These results are consistent with the combined results from the meta-analysis (Figure 10).

In summary, there were no significant differences found in the symmetry index of either temporal or spatial asymmetries between the socket designs.

\subsubsection{Step width}

Step width is a measure of the medio-lateral separation of the feet. Yigiter et al. was the only study in this review to analyse step width. ${ }^{55}$ Twenty participants walked the 12metre laboratory walkway at self-selected speed, with step width recorded from the central seven metres to ensure constant velocity. Four measurements of step width were recorded as the horizontal distance between the two heels. The authors reported a statistically significant $(p<0.05)$ difference in step width, with the step width of those using the TSB socket design narrower, with a mean of $10.5 \mathrm{~cm}(S D=2.3 \mathrm{~cm})$ compared to the SSB socket design, with a mean of $14.0 \mathrm{~cm}(S D=2.2 \mathrm{~cm})$. The Wilcoxon Rank Test and Pearson's Correlation Analysis were used in statistical evaluations, with the alpha level set at 0.05 . Decreased step width indicates a more efficient forward progression as the centre of mass does not exhibit as much lateral displacement. Individuals with poor balance often display a wider step width, or rely on handrails or rehabilitation gait aids to improve stability when walking. It can be concluded that the TSB socket design offers a more intimate fit and therefore reduces lateral movement and improves balance during ambulation.

\subsubsection{Balance}

Yigiter et al. was the only study in this review to investigate balance. ${ }^{55}$ The 20 subjects were required to stand on the prosthetic side with eyes open for 30 seconds and then with eyes closed. Balance is a complex process involving coordination of multiple sensory, motor and biomechanical components. An individual senses the position of his or her body in relation to gravity and their surroundings by combining visual, vestibular and somatosensory inputs. It is commonly believed that musculoskeletal injury to one or both of the lower extremities alters the somatosensory (proprioceptive) input that is 
essential for neuromuscular coordination. ${ }^{49}$ Testing with eyes closed eliminates the visual input in balance. Body oscillations and compensatory motions were observed and time taken to commence compensatory motions was recorded in seconds.

Balance on the prosthetic side with eyes open showed a statistical significance $(p<0.05)$ in favour of the TSB socket design, with a mean of 17.8 seconds $(S D=5.6)$ compared to the SSB design with a mean of 13.3 seconds $(S D=6.2)$.

\subsubsection{Weight bearing}

Yigiter et al. was the only study to investigate the weight bearing characteristics of socket designs with their 20 study participants. ${ }^{55}$ Gruendel's method was used which was developed to assess weight bearing (WB) through hemiplegic legs. ${ }^{50}$ Minimal (min WB) and maximal (max WB) weight-bearing values are recorded. The formula (Max WB $+\min$ WB)/2 was used to provide average weight bearing (M1) of the compromised limb. The percentage of total weight borne through the compromised limb is calculated using the formula M1 / TBW $\times 100$. This method can be used with any condition where there is unequal weight acceptance. Percentage of weight bearing through the prosthetic limb was recorded, while the subject was standing on two juxtaposed scales for three consecutive minutes.

The authors reported a statistically significant $(p<0.05)$ increase in the percentage of body weight being transferred through the amputated limb, with the TSB socket design having a mean of $42.6 \%(S D=3.2)$ compared with the mean for the SSB socket of $38.0 \%(S D=3.9)$.

\subsubsection{Activities of daily living (ADLs)}

Activities of daily living (ADLs) are tasks chosen to simulate day-to-day functional activities. Two studies of experimental design assessed ADLs within their study populations. ${ }^{52,55}$ Selles et al. was a prospective RCT, 52 and Yigiter et al. a quasiexperimental before and after study.55 The ADLs observed and reported on and the methodology utilised in the data gathering process were sufficiently different, preventing the pooling of data from these two studies in statistical meta-analysis.

Selles et al.'s study included a sample of 26 amputees with amputations due to various aetiologies: including $50 \%$ of traumatic origin; all participants had been walking with a prosthesis for at least 12 months and all subjects had been wearing the SSB socket 
design with foam interface and proximal suspension prior to recruitment and for the baseline ADL evaluation. ${ }^{52}$ The mean age of participants in this study was 63 years $(S D=14.5)$. The study population was randomised into two groups, one receiving a TSB socket with gel interface and pin suspension and the other an SSB socket design with gel interface and pin suspension. This was the only study in this review where both the comparator and intervention groups utilised the same type of suspension and interface with the new socket designs, ensuring that socket design was the only true variation. ADL analysis was repeated after three months use with the new TSB sockets.

The authors analysed ADLs through mobility-related activities including body postures, body motions and the transitions between body postures as assessed via an activity monitor. The activity monitor is a validated tool that has been used in previous transtibial prosthetic studies. ${ }^{51}$ Subjects were asked to wear the monitor for two days, where data from a 24-hour period was used. Subjects were given limited information about the monitor in order to limit any possible influence on the outcome.

Time spent walking, measured in hours, was lower with the new TSB sockets 4.61 hours $(S D=2.40)$ at baseline, and a mean of 4.55 hours $(S D=3.82)$ at follow-up when compared to the group receiving the new SSB sockets recording a mean of 7.43 hours $(S D=4.76)$ at baseline and 5.25 hours $(S D=4.54)$ at follow-up.

Time spent upright (in hours) was significantly higher at baseline in the SSB group, with a mean of 20.02 (SD=9.51); however this was reduced at follow-up with a mean of 16.77 (SD=8.76); whereas the TSB group increased their time spent upright with a mean of 13.11 ( $S D=4.98)$ hours at baseline, compared to a mean of 14.42 hours $(\mathrm{SD}=7.62)$ at follow-up.

The number of walking periods recorded for the TSB group remained the same at baseline $(M=119.18, S D=62.30)$ compared to follow-up $(M=119.09, S D=87.07)$; however the SSB group showed a reduced number of walking periods at follow-up, with a mean of $124.38(S D=91.05)$, compared to the baseline measurement mean of 186.33 $(S D=114.73)$.

The number of body posture transitions were comparable between the two groups, with the TSB group increasing from baseline, with a mean of $118.45(S D=50.33)$ to followup, with a mean of 122.09 (SD=51.92); and the SSB group also increasing from 
baseline with a mean of $130.33(S D=45.24)$ to follow-up with a mean of 132.85 $(S D=50.17)$.

Overall, time (as a percentage of 24hours) spent in dynamic activities increased with the TSB group, with a mean of $6.35 \%(S D=3.49)$ at baseline compared to $6.66 \%$ $(S D=4.77)$ at follow-up. The SSB group showed a decrease from baseline with a mean of $9.00 \%$ ( $S D=5.61)$ to follow-up, with a mean of $7.44 \%$ (SD=6.17).

Yigiter et al.'s study was unique in that all 20 subjects were new amputees engaged in primary rehabilitation. ${ }^{55} \mathrm{~A}$ number of facility-specific in-house outcome measures were used to gather data for ADLs; however these were not valid for comparison with other studies and are therefore presented separately.

ADLs were evaluated in terms of the time required to complete certain ambulatoryassociated tasks in the rehabilitation unit. These included: donning and doffing the prosthesis, climbing and descending 10 steps, walking up and down a six-metre incline of 40 degrees, picking up an object from the floor, crossing an obstacle of $20 \mathrm{~cm}$ in height and $30 \mathrm{~cm}$ in depth and sitting down and standing up from a chair.

TSB was the better performing socket with all activities assessed. The time taken to don the prosthesis was a mean of 6.8 seconds $(S D=1.5)$ with the TSB design, compared to a mean of 8.6 seconds $(S D=2.0)$ with the $S S B$ socket. Doffing the prosthesis was significantly quicker with the TSB socket, with participants taking a mean of 2.2 seconds $(S D=0.9)$ compared to the $S S B$ design with participants taking a mean of 4.2 seconds $(S D=0.9)$. Climbing up stairs was also performed more efficiently with the TSB design, taking an average of 10.5 seconds $(S D=1.1)$ compared to 14.4 seconds (SD=1.1) with the SSB socket; and similar was found in descending 10 stairs, with the TSB design at a mean of 8.8 seconds $(S D=1.1)$ compared to the $S S B$ socket with a mean of 11.3 seconds (SD=1.4). Those wearing the TSB socket design were on average quicker in ascending $(M=10.5$ seconds, $S D=2.7)$ and descending $(M=11.6$ seconds, $S D=2.2$ ) the incline compared to those wearing the $S S B$ design, timed on average at 13.7 seconds $(S D=3.7)$ and 14.3 seconds $(S D=3.4)$, respectively. Picking up an object from the floor was done on average more quickly in those wearing the TSB socket design, taking a mean of 1.4 seconds $(S D=0.4)$ compared to those wearing the SSB design that were timed at an average of 1.5 seconds $(S D=0.4)$. Each of these results were statistically significant with $p<0.05$. 
Those wearing the TSB design also showed better performance in crossing an obstacle $(M=1.2$ seconds, $S D=0.4)$ compared to those wearing the SSB socket $(M=1.3$ seconds, $S D=0.4)$ and sitting down and standing up from a chair was also performed more quickly among the participants wearing the TSB socket design with a mean of 1.4 seconds $(S D=0.4)$ compared to those wearing the SSB design with a mean of 1.5 seconds $(S D=0.4)$.

In summary, evaluating ADL tasks often has greater meaning to the rehabilitation team and persons with an amputation than other outcome measures commonly evaluated in research, as they represent tasks important to enable a level of independence in everyday living. Results of both the Selles et al. and Yigiter et al. studies indicate improved performance in a range of practical tasks and postures with the TSB socket design. ${ }^{52,55}$ It is important that the interface and suspension method used in the prostheses worn by the 26 participants in Selles et al.'s study were identical, as this suggests that the conclusion may be drawn that an increase in functional performance in their study was likely due to the difference in socket design.

3.5.2. Function and mobility - Community based physical performance measures

In addition to the ADL measures reported by Selles et al. step activity and prosthetic wear time were also analysed in the community setting.52 The home-based or community environment is important as data collected here is typically gathered over a longer timeframe, with subjects participating in their normal daily routine whilst being monitored.

\subsubsection{Step count}

Step count is the number of steps taken per day, measured using a count device. Two studies recorded steps per minute per day in the community setting through use of an activity monitor attached to the prosthesis. ${ }^{23,51}$

Both Klute and Coleman used the step watch activity monitor (SAM) to record step count. ${ }^{51,23}$ The SAM collects data relating to the number of steps taken per minute by an individual and is programmed by the researcher in relation to the participants' individual physical characteristics including height, gait pattern and variance of cadence, which is used to ensure accuracy of data collection. Laboratory testing before 
commercial distribution of the SAM illustrates that when used correctly, accuracy typically exceeds $99 \% .{ }^{24}$ The SAM only collects the number of steps for the leg to which it is attached.

Coleman et al. applied the SAM to the prosthetic limb in the final two weeks of each socket design's three-month study period. ${ }^{23}$ Klute also applied the SAM to the prosthetic limb for the last two weeks of each socket design's three-week study period.51 Both studies recorded steps per minute for the continuous two-week period. Participant details and results are presented in Table 8 below, with pooled data presented in a meta-analysis (Figure 11).

Table 8: Studies included in a meta-analysis reporting steps per day (1000's).

\begin{tabular}{|c|c|c|c|c|}
\hline Study ID & Study design & Participants & $\begin{array}{l}\text { Intervention/ } \\
\text { comparator }\end{array}$ & $\begin{array}{l}\text { Steps/day (1000's) } \\
\text { mean (SD) }\end{array}$ \\
\hline $\begin{array}{l}\text { Coleman } \\
2004^{23}\end{array}$ & & $\begin{array}{l}\mathrm{n}=13 \\
\text { Aetiology: V/T/O (0/13/0) }\end{array}$ & $\begin{array}{l}\text { TSB: GI, PS } \\
\text { SSB: FI, SC-S }\end{array}$ & $\begin{array}{l}\text { TSB } 2.262(1.39) \\
\text { SSB } 4.135(1.39)\end{array}$ \\
\hline $\begin{array}{l}\text { Klute } \\
2011^{51}\end{array}$ & $\begin{array}{l}\text { Experimental } \\
\text { RCT }\end{array}$ & $\begin{array}{l}\mathrm{n}=5 \\
\text { Aetiology: V/T/O (1/4/0) }\end{array}$ & $\begin{array}{l}\text { TSB: GI, VS } \\
\text { SSB: GI, PS }\end{array}$ & $\begin{array}{l}\text { TSB } 2.714(0.64) \\
\text { SSB } 5.214(1.29)\end{array}$ \\
\hline
\end{tabular}

Abbreviation: TSB: Total surface bearing; SSB: Specific surface bearing; GI: Gel Interface; FI: Foam Interface; PS: Pin Suspension; SC-S: Supracondylar suspension; VS: Vacuum suspension; Aetiology= V: Vascular, T: Traumatic, O: Other; Yrs: Years; SD: Standard deviation.

In total, there were 36 participants with measured step count per day. The metaanalysis shows an effect size of (WMD=-2.14; $C l=-2.95,-1.32)$ using a random effects model. There was no statistical heterogeneity found between the studies (Chi square $=0.55, p=0.46,12=0 \%$ ). A statistically significantly higher step count per day was recorded with the SSB socket design $(p<0.00)$, with an effect size of -2.14 . 


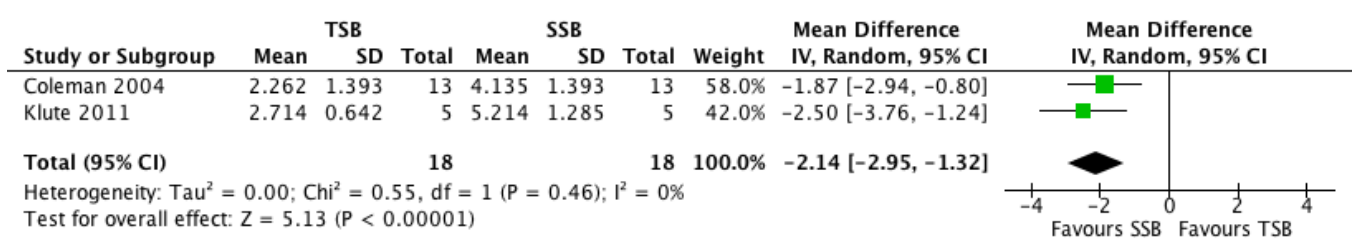

Figure 11: Two experimental studies comparing the TSB socket design with the SSB socket design in terms of step count

\subsubsection{Prosthetic wear time}

Prosthetic wear time was reported by Coleman et al. and measured through use of the SAM, as described in the previous section (i.e. step count). ${ }^{23}$ When the data was downloaded from the SAM, the number of steps taken in each 24 hour period of wear was shown, in addition to the amount of time the person spent at rest and in low, medium and high activity levels.

The authors report that subjects spent $82 \%$ more time wearing the SSB socket design compared with the TSB design (13.3 vs. 7.3 hours/day; $p=0.002$ ); and $72 \%$ more time doing high intensity activity ( $>30$ steps / minute) (27.1 vs. 15.8 minutes/day; $p=0.002$ ). Results were analysed using a paired samples two-tailed Student's t-test. The authors suggest that increased wear time and intensity of activity with the SSB socket suggests a preference for use and an increased comfort with the SSB socket design.

Potential confounds to this interpretation of the results include that subjects were allowed to revert back to their pre-study prosthesis, even though they were asked to wear the study sockets for as much of the time as possible. Actual ambulation was only recorded with the study socket. If the subject chose to switch to the previous design during the day, those steps were not recorded and hence the wear time was not recorded. The authors describe that the difference in overall activity reflects difference in amount of time the subjects wore the study sockets. It is reported that the SSB socket was worn for $86 \%$ of the days monitored, whereas the TSB socket was worn more sporadically, with some partial days and some entire days skipped; it was worn for the full day in only $55 \%$ of the days monitored.

In summary, increased function, as measured by wear time and ambulation activity (using the SAM) was found with the SSB system; however these results should be interpreted with caution due to confounding factors. 


\subsubsection{Function and mobility: Questionnaire}

A single self-report questionnaire subscale titled 'Ambulation' within the Prosthesis Evaluation Questionnaire (PEQ) was used to assess the domain of function and mobility. ${ }^{35}$ The PEQ was specifically designed for lower limb prosthetic use and consists of 15 subscales that examine an amputee's perception of the quality of their prosthesis, their ability to perform various activities when using their prosthesis and the psychological and social effects of living with the prosthesis. Essentially, it is a measure of prosthesis-related quality of life. ${ }^{35}$

The questions in the PEQ relate to the four weeks preceding the assessment date. The reliability, validity and sensitivity of the test have all been investigated and results identify the PEQ as appropriate for use among lower limb amputees. However, only nine of the sub-scales have been validated, of which the ambulation subscale is one.

\subsubsection{Ambulation}

'Ambulation' is a validated sub-scale of the PEQ, which was used in three of the included studies.23,50,51 This subscale is assessed through participants answering eight questions with response in a Visual Analogue Scale (VAS) of $100 \mathrm{~mm}$. Questions in the Ambulation sub-scale query the amputee's general ability to walk, as well as in confined spaces, on stairs and ramps, in urban environments and on slippery surfaces. The scale is recorded such that 100 indicates the best outcome, for example 'easiest to walk' and 0 indicates the 'most difficult to walk'. Participant characteristics and results are detailed in Table 9, with results combined in meta-analysis (Figure 12). 
Table 9: Studies included in meta-analysis assessing ambulation

\begin{tabular}{|c|c|c|c|c|}
\hline Study ID & Study design & Participants & $\begin{array}{l}\text { Intervention/ } \\
\text { comparator }\end{array}$ & $\begin{array}{l}\text { Ambulation } \\
\text { mean (SD) 0-100 }\end{array}$ \\
\hline $\begin{array}{l}\text { Coleman } \\
2004^{23}\end{array}$ & & $\begin{array}{l}n=13 \\
\text { Aetiology: V/T/O (0/13/0) }\end{array}$ & $\begin{array}{l}\text { TSB: GI, PS } \\
\text { SSB: FI, SC-S }\end{array}$ & $\begin{array}{l}\text { TSB } 71.6(24) \\
\text { SSB } 78.6(17.5)\end{array}$ \\
\hline $\begin{array}{l}\text { Engsberg } \\
2006^{50}\end{array}$ & Experimental RCT & $\begin{array}{l}\mathrm{n}=43 \\
\text { Aetiology: V/T/O (unknown) }\end{array}$ & $\begin{array}{l}\text { TSB } \\
\text { SSB }\end{array}$ & $\begin{array}{l}\text { TSB } 81(21) \\
\text { SSB } 83(17)\end{array}$ \\
\hline $\begin{array}{l}\text { Klute } \\
2011^{51}\end{array}$ & & $\begin{array}{l}n=5 \\
\text { Aetiology: V/T/O (1/4/0) }\end{array}$ & $\begin{array}{l}\text { TSB: GI, VS } \\
\text { SSB: GI, PS }\end{array}$ & $\begin{array}{l}\text { TSB } 67(22) \\
\text { SSB } 95(6)\end{array}$ \\
\hline
\end{tabular}

Abbreviation: TSB: Total surface bearing; SSB: Specific surface bearing; GI: Gel interface; FI: Foam interface; PS: Pin suspension; SC-S: Supracondylar suspension; VS: Vacuum suspension; Aetiology= V: Vascular, T: Traumatic, O: Other; Yrs: Years; SD: Standard deviation

In total, there were 122 participants who were assessed using the PEQ ambulation subscale. The meta-analysis shows an effect size of $(\mathrm{WMD}=-10.03 ; \mathrm{Cl}=-23.88,3.81)$ using a random effects model. High statistical heterogeneity was found between the studies (Chi square $=5.61, p=0.06,1^{2}=64 \%$ ). There was no statistically significant difference $(p=0.16)$ in self-reported ambulation between socket designs.

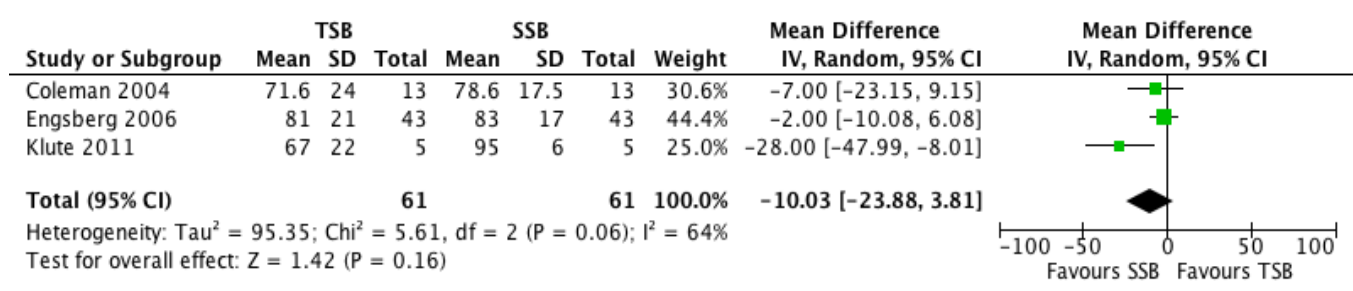

Figure 12: Three experimental studies comparing the TSB and SSB socket designs in terms of self-reported ambulation

Ambulation was the only self-report measure included in this meta-analysis to assess function and mobility. The importance of this measure is that it allows for assessment of how the prosthetic user believes their walking abilities to be and their confidence walking in different environments and situations. Klute ${ }^{51}$ differed from Coleman ${ }^{23}$ and Engsberg 50 as the TSB design included in their study used vacuum suspension, which can be difficult to tolerate. It is important to also consider that only five of the 20 
subjects recruited by Klute completed the protocol due to intolerance of the TSB socket with vacuum.

Overall for the function and mobility domain, community-based measures indicate a preference for the SSB design, although potential confounds have been highlighted; laboratory-based measures indicate a preference for the TSB design, particularly when using gel interface liners with pin suspension mechanism.

\subsubsection{Comfort and pain: questionnaires}

Comfort and pain was determined through results from six self-report questionnaires. 'Residual Limb Health' is a validated sub-scale of the PEQ,35 Socket Comfort Score (SCS), ${ }^{36}$ Brief Pain Inventory (BPI), ${ }^{46}$ and 'Wear comfort', with the last two 'Pain' and 'Phantom pain' measured via non-validated sub-scales of the PEQ.

\subsubsection{Residual Limb Health}

Three studies $23,50,51$ examined Residual Limb Health (RLH) as measured using a validated sub-scale of the PEQ. ${ }^{35}$ The RLH sub-scale has eight questions examining sweat, smell, volume change, rashes, in-grown hairs and blisters. All three studies were RCTs of cross-over design, in which the PEQ subscale was completed by subjects after having used each socket design for a minimum of four weeks. Each participant compared both sockets, i.e. the studies were of a within-subjects design. Population characteristics and outcome results are presented in Table10. 
Table 10: Studies within the meta-analysis assessing the 'Residual Limb Health' subscale of the PEQ.

\begin{tabular}{|c|c|c|c|c|}
\hline Study ID & Study design & Participants & $\begin{array}{l}\text { Intervention/ } \\
\text { comparator }\end{array}$ & $\begin{array}{l}\text { RLH } \\
\text { mean (SD) 0-100 }\end{array}$ \\
\hline $\begin{array}{l}\text { Coleman } \\
2004^{23}\end{array}$ & \multirow{3}{*}{ Experimental RCT } & $\begin{array}{l}\mathrm{n}=13 \\
\text { Aetiology: V/T/O (0/13/0) }\end{array}$ & $\begin{array}{l}\text { TSB: GI, PS } \\
\text { SSB: FI, SC-S }\end{array}$ & $\begin{array}{l}\text { TSB } 65.1(19.8) \\
\text { SSB } 64.5(23.1)\end{array}$ \\
\hline $\begin{array}{l}\text { Engsberg } \\
2006^{50}\end{array}$ & & $\begin{array}{l}\mathrm{n}=43 \\
\text { Aetiology: V/T/O (unknown) }\end{array}$ & $\begin{array}{l}\text { TSB } \\
\text { SSB }\end{array}$ & $\begin{array}{l}\text { TSB } 76(17) \\
\text { SSB } 73(17)\end{array}$ \\
\hline $\begin{array}{l}\text { Klute } \\
2011^{51}\end{array}$ & & $\begin{array}{l}n=5 \\
\text { Aetiology: V/T/O (1/4/0) }\end{array}$ & $\begin{array}{l}\text { TSB:GI, VS } \\
\text { SSB: GI, PS }\end{array}$ & $\begin{array}{l}\text { TSB } 77(20) \\
\text { SSB } 90(5)\end{array}$ \\
\hline
\end{tabular}

Abbreviation: TSB: Total surface bearing; SSB: Specific surface bearing; GI: Gel interface; FI: Foam interface; PS: Pin suspension; SC-S: Supracondylar suspension; VS: Vacuum suspension; Aetiology= V: Vascular, T: Traumatic, O: Other; Yrs: Years; SD: Standard deviation

Klute et al. did not perform statistical analysis on the PEQ results due to the small sample size in their study; however data has been extracted and combined with data from the other two studies in meta-analysis. ${ }^{51}$

There were 122 participants for whom data on limb health was measured using the 'Residual Limb Health' subscale of the PEQ. The meta-analysis of data showed an effect size of $(\mathrm{WMD}=-0.28 ; \mathrm{Cl}=-8.45,7.89)$ using a random effects model. There was low statistical heterogeneity found between the studies (Chi square $=2.60, p=0.27$, $1^{2}=23 \%$ ). There was no statistically significant difference in the residual limb health of

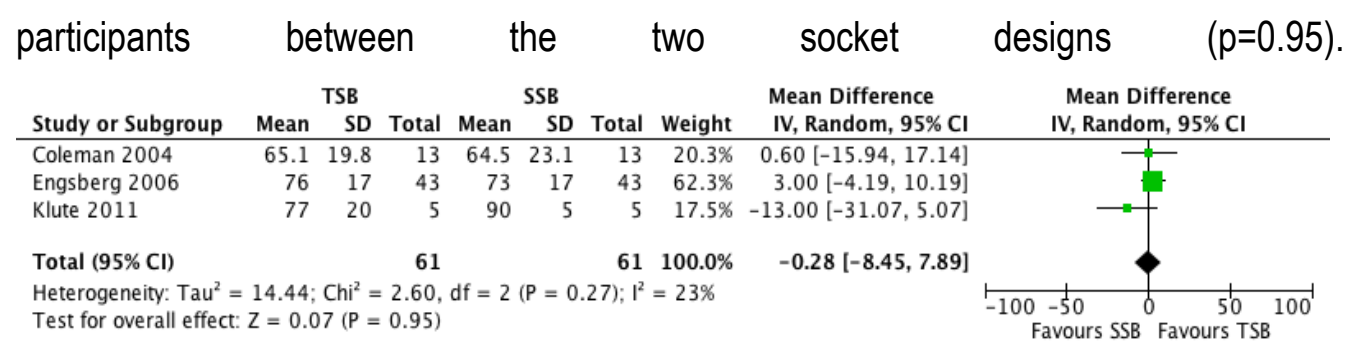

Figure 13: Three experimental studies comparing the TSB and SSB socket designs in terms of residual limb health 
Clinically, an improvement in residual limb health with the TSB design would be expected due to a lack of specific loading and greater surface area distribution for weight bearing. However this is not reflected in the pooled data.

\subsubsection{Socket Comfort Score}

The Socket Comfort Score (SCS) is a single-question self-report tool using an 11-point numeric rating scale, validated specifically for assessment of socket fit comfort. ${ }^{36}$ Subjects are asked to rate the comfort of their socket fit by circling a number from 0 to 10 , where 0 is the 'most uncomfortable' and 10 is 'completely comfortable'.

Three studies assessed comfort through use of the SCS. ${ }^{23,24,53}$ Incomplete data precluded one study from being included in the meta-analysis. ${ }^{24}$ Participant characteristics and outcomes of two studies are presented in Table 11 and results combined in meta-analysis (Figure14). ${ }^{23,53}$

Table 11: Studies included in the meta-analysis that included the Socket Comfort Score

\begin{tabular}{|l|l|l|l|l|}
\hline Study ID & Study design & Participants & $\begin{array}{l}\text { Intervention/ } \\
\text { comparator }\end{array}$ & $\begin{array}{l}\text { SCS } \\
\text { mean (SD) } \mathbf{0}-10\end{array}$ \\
\hline $\begin{array}{l}\text { Coleman } \\
\mathbf{2 0 0 4}{ }^{23}\end{array}$ & $\begin{array}{l}\text { Experimental } \\
\text { RCT }\end{array}$ & $\mathrm{n}=13$ & TSB: GI, PS & TSB $6.84(3.00)$ \\
\hline $\begin{array}{l}\text { Manucharian } \\
\mathbf{2 0 1 1 ^ { 5 3 }}\end{array}$ & $\begin{array}{l}\text { Experimental } \\
\text { Q-RCT }\end{array}$ & $\mathrm{n}=36$ & SSB: FI, SC-S & SSB $7.23(2.50)$ \\
\hline
\end{tabular}

Abbreviation: TSB: Total surface bearing; SSB: Specific surface bearing; FI: Foam interface; SIvS: Sleeve and valve suspension; SCS: Socket Comfort Score; Aetiology= V: Vascular, T: Traumatic, O: Other; Yrs: Years; SD: Standard deviation

In total, there were 62 participants reporting on comfort as measured by the Socket Comfort Score questionnaire. The meta-analysis shows an effect size of (WMD=-1.55; $\mathrm{Cl}-3.40,0.31)$ using a random effects model. A high level of statistical heterogeneity was detected between the studies (Chi square=2.31, $p=0.13,1^{2}=57 \%$ ), however a preference was shown towards the SSB socket design with a small effect size of -1.55 , $p=0.10$; this was not statistically significant. 


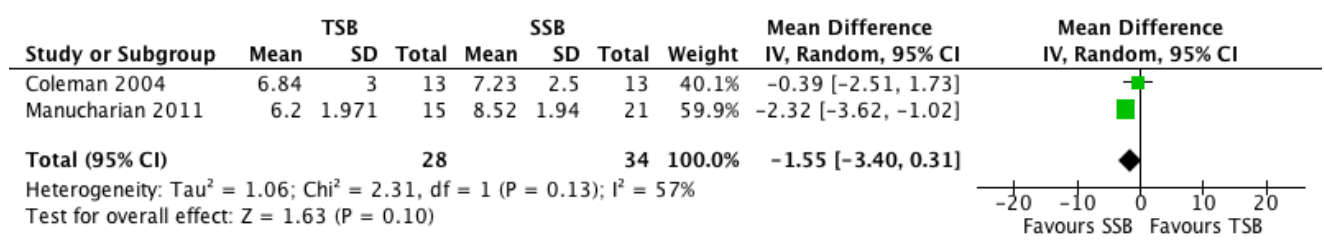

Figure 14: Two experimental studies comparing TSB socket design with SSB socket design in terms of socket comfort

Datta et al. ${ }^{24}$ also assessed comfort using the Socket Comfort Score (SCS). ${ }^{36}$ Eleven subjects in the experimental group reported a mean of 7.2 (range: 5-9) at baseline with the SSB socket and 8.2 (range: 6-10) using the TSB socket. While this indicates an improvement in comfort with the TSB socket design, results could not be included in the meta-analysis, as only range values were provided without SDs and the results for the baseline and follow-up with the SSB control group were not reported. Authors were unable to be contacted for clarification.

SCS was used by three studies to assess comfort of the sockets; however one had reported incomplete results. Manucharian chose to assess only comfort in his study, considering use of five outcome measures before deciding on SCS to best reflect prosthetic users' experience of comfort and pain, derived from prosthetic wear. ${ }^{53}$ However, in an attempt to eliminate potential confounds, prosthetic sockets in his study were fabricated using foam interfaces for both the TSB and SSB designs. This is the only study in this review reporting on use of a foam interface with the TSB design, which is not widely used in clinical practice. The very nature of a non-specific loading on the residuum, as in the TSB style, relies on the uniform compression of soft tissue, which is achieved through the roll-on of the silicone or gel interface. It is clinically understandable that a foam interface without specific load bearing would be assessed as low comfort. Statistically, there was no difference between the two designs and this is further reinforced by the previously mentioned clinical perspective.

\subsubsection{Brief Pain Inventory}

Coleman et al. ${ }^{23}$ reported using a modified version of the Brief Pain Inventory (BPI). ${ }^{46}$ The BPI is an 11-item questionnaire that consists of four questions utilising a 0-10 numeric rating scale asking patients to rate their pain at its 'worst in the last 24-hours', 'least in the last 24-hours', 'pain on average', and 'pain right now', with zero indicating 'no pain' and 10 representing 'pain as bad as you could imagine'. The remaining seven $\mathrm{BPI}$ questions probe the degree to which the pain interferes with general activity, mood, 
walking ability, normal work, relations with other people, sleep and enjoyment of life, again using a 0-10 numeric rating scale. For these interference items, zero represents 'does not interfere' and 10 indicates 'interferes completely'. ${ }^{52}$ Coleman et al. stated that the BPI results were analysed using the Chi-square distribution test. Mean and SD pain scale data were not reported; only statistical comparison results ( $p$ values) were reported. ${ }^{23}$ No difference was found in any of the $11 \mathrm{BPI}$ questions with $p$ values ranging from $(p=0.67$ to $p=0.99)$, indicating no difference in the experience and reporting of pain comparing the TSB and SSB socket designs.

\subsubsection{Wear comfort}

Datta et al. ${ }^{56}$ reported on the 'wear comfort' of the TSB and SSB sockets via the use of a self-report questionnaire. The 3-page composite questionnaire was devised by the authors to gather information relating to personal experience with new material, technique in regard to function, comfort and pain and ease of use. Due to the fact that the instrument developed was not a standardised tool, the resultant data was not pooled with other studies; however results are provided in narrative form.

The authors used this questionnaire in their descriptive study of retrospective case series with 54 subjects, who completed the postal questionnaire relating to their experience using the traditional SSB socket with foam interface and supracondylar suspension and compared to the recently provided TSB socket design with gel interface and pin lock suspension. The mean age of participating subjects was 48.35 years (range 22-80 years). Causes of amputation within this group included: 27 traumatic, 11 vascular, six congenital and 10 miscellaneous/undisclosed.

Comfort of the prosthesis was derived from the returned questionnaire; where responses were recorded using a digital scale with a range of zero to five. Zero represents 'very poor' and five represents 'very good'. Authors reported responses from 52 of the 54 returned questionnaires in relation to 'wear comfort' with the TSB socket, with a mean score of 3.50 . Fifty-one responses were received from the 54 returned questionnaires in response to wear comfort with the SSB socket, with a mean score of 2.82. Statistical analysis was conducted using the Wilcoxon matched-pairs signed ranks test; with no statistically significant difference $(p=0.68)$ found in reported comfort between the two socket designs. 


\subsubsection{Pain}

Pain, like comfort is a very subjective symptom. Selles et al. ${ }^{52}$ explored the experience of pain in 26 subjects through the use of the Dutch version of the PEQ. Pain is one of the 15 subscales used in the PEQ, however it is non-validated. ${ }^{35}$ There are 16 questions in the pain sub-scale that assess general pain, residual limb pain, phantom pain, contra-lateral limb pain and back pain. As this sub-scale has not been validated, the questions could not be grouped together and are reported individually. Selles et al. chose one question relating to residual limb pain to use in their study. A $100 \mathrm{~mm}$ visual analogue scale was used to assess pain experience, with zero being 'extremely intense' and 100 being 'extremely mild'.

The authors reported no difference $(\mathrm{p}=0.47)$ in pain between the two socket designs, with results for the TSB group at 82.1 (SD=29.0) compared to the SSB group at 90.8 $(S D=15.4)$ measured at follow-up after subjects had worn newly provided sockets after three-months acclimatisation.

\subsubsection{Phantom pain}

Selles et al. chose one question from the PEQ ${ }^{35}$ to assess subjects' experience with phantom pain. ${ }^{22} \mathrm{~A} 100 \mathrm{~mm}$ visual analogue scale was used to assess pain experience, with zero being 'extremely intense' and 100 being 'extremely mild'. This scale has also not been validated. Authors did not find any difference in reported phantom pain between the two socket designs ( $p=0.77)$, with results for the TSB group at 73.8 $(S D=33.3)$ compared to the $S S B$ group at $74.4(S D=32.8)$.

In summary, subjects who reported on both residual limb pain and phantom pain, found little difference in pain levels regardless of which socket design they were wearing.

\subsubsection{Quality of Life}

Quality of Life (QoL) was assessed by eight studies using nine different measures, all of which were in the self-report format. Frustration with the prosthesis was measured in three studies;23,50,51 and utility, sounds, social burden, perceived response and appearance were all assessed in two studies. ${ }^{23,50}$ All of these measures are validated sub-scales of the PEQ. ${ }^{35}$ Questions within each sub-scale are grouped together for the purpose of analysis and the overall score is presented for each measure. The PEQ subscale satisfaction, which consists of three individual questions, was used in only one of 
the included studies. ${ }^{52}$ Socket preference was asked of participants in seven studies; whereby indication of which prosthesis was preferred and which system subjects would choose to keep was recorded. 23,48a,48b,50,52,54,55

\subsubsection{Socket preference}

Seven studies asked participating subjects which socket design they preferred and would choose to keep at the conclusion of the study. ${ }^{23,48 a, 48 b, 50,52,54,55}$ Coleman et al. asked subjects which of the TSB and SSB sockets they would prefer to keep, with 12 subjects selecting a newly provided socket (nine SSB and three TSB) and one preferring to retain their pre-study SSB prosthesis. ${ }^{23}$ While Astrom et al. reported that all seven subjects chose to keep and use their recently provided TSB prosthesis. ${ }^{54}$ Selles et al. collected Yes/No responses for socket preference, with 13 participants selecting to keep the SSB design and 11 the TSB design. ${ }^{52}$ Engsberg et al. reported on sockets kept for primary use, with 41 choosing a preferred socket and two subjects opting to keep both sockets using the SSB design for sedentary activities and the TSB socket for exercising. ${ }^{50}$ Hachisuka asked both study groups to rate on a five-point Likert scale their level of satisfaction with the socket designs; 'satisfied' and 'somewhat satisfied' were determined as indicating preference for a given design, while 'somewhat dissatisfied', 'dissatisfied' and 'undetermined' indicated that that particular design was not preferred. ${ }^{48 a, 48 b}$ Yigiter et al. recorded the socket design subjects chose to be discharged from the rehabilitation centre with. ${ }^{55}$ Data is presented in Table 12, together with participant characteristics. 
Table 12: Studies included in narrative commentary assessing socket preference

\begin{tabular}{|l|l|l|l|l|}
\hline Study ID & Study design & Participants & $\begin{array}{l}\text { Intervention/ } \\
\text { comparator }\end{array}$ & Socket preference \\
$\mathbf{x} / \mathbf{n}$
\end{tabular}

Abbreviation: TSB: Total surface bearing; SSB: Specific surface bearing; GI: Gel interface; FI: Foam interface; PS: Pin suspension; SI-vS: Sleeve and valve suspension; SC-S: Supracondylar suspension; VS: Vacuum suspension; Aetiology= V: Vascular, T: Traumatic, O: Other; Yrs: Years; SD: Standard deviation

Data was explored using a meta-analysis with odds ratio resulting in a large effect size of $3.66(\mathrm{Cl} 0.86,15.50 ; \mathrm{p}=0.08)$ using a random effects model. However, there is substantial statistical heterogeneity between the studies, as indicated by the wide confidence intervals (Chi square $=27.26, p=0.0001,{ }^{2}=78 \%$ ) 


\begin{tabular}{|c|c|c|c|c|c|c|c|c|}
\hline Study or Subgroup & \multicolumn{2}{|l|}{ TSB } & \multicolumn{2}{|c|}{ SSB } & Weight & Odds Ratio & \multicolumn{2}{|c|}{$\begin{array}{c}\text { Odds Ratio } \\
\mathrm{M}-\mathrm{H}, \text { Random, } 95 \% \mathrm{Cl}\end{array}$} \\
\hline Astrom 2004 & 7 & 7 & 0 & 7 & $8.0 \%$ & $225.00[3.93,12894.87]$ & & $\longleftrightarrow$ \\
\hline Coleman 2004 & 3 & 13 & 10 & 13 & $16.0 \%$ & $0.09[0.01,0.56]$ & & \\
\hline Engsberg 2006 & 25 & 43 & 16 & 43 & $20.0 \%$ & $2.34[0.99,5.57]$ & & \\
\hline Hachisuka 1998 & 15 & 23 & 8 & 23 & $18.7 \%$ & $3.52[1.04,11.83]$ & & 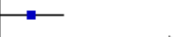 \\
\hline Hachisuka B & 9 & 9 & 0 & 9 & $8.1 \%$ & $361.00[6.47,20143.55]$ & & + \\
\hline Selles 2005 & 11 & 12 & 13 & 14 & $11.6 \%$ & $0.85[0.05,15.16]$ & & \\
\hline Yigiter 2002 & 15 & 20 & 5 & 20 & $17.8 \%$ & $9.00[2.15,37.66]$ & & - \\
\hline Total $(95 \% \mathrm{CI})$ & & 127 & & 129 & $100.0 \%$ & $3.66[0.86,15.50]$ & & \\
\hline Total events & 85 & & 52 & & & & & \\
\hline $\begin{array}{l}\text { Heterogeneity: } \mathrm{Tau}^{2}= \\
\text { Test for overall effect }\end{array}$ & $\begin{array}{l}2.52 ; C h \\
Z=1.76\end{array}$ & $\begin{array}{l}i^{2}=27 \\
(P=0\end{array}$ & $\begin{array}{l}.26, \mathrm{df}= \\
.08)\end{array}$ & $=6(P$ & $=0.0001)$ & $; I^{2}=78 \%$ & $\begin{array}{cc}0.001 & 0.1 \\
\text { Favours SSB } & 1\end{array}$ & $\begin{array}{cc}10 & 1000 \\
\text { Favours TSB }\end{array}$ \\
\hline
\end{tabular}

Figure 15: Socket preference (Seven experimental studies comparing TSB socket design with SSB socket design)

Results from the seven experimental studies showed that $67 \%$ of participants who trialled the TSB socket indicated their preference for this design. $54,23,50,488,486,52,55$ Regarding the SSB socket, $40 \%$ of participants indicated a preference for this design. ${ }^{23,50,88 a, 52,55}$ Some participants selected both socket designs without a preference for either one specifically. 50

As with walking speed, stride length and cadence, the type of interface or suspension method may confound the estimate of effect size of socket preference. Therefore subgroup analysis was conducted to ascertain if results were different when controlling for these potential confounds. The four studies included in this sub-group analysis all utilised a gel interface with distal pin suspension in the TSB design and used a foam interface with supracondylar suspension in the SSB socket design. $23,48 a, 48,55$ The results are combined below in meta-analysis (Figure 16).

Overall there were 130 participants reporting on socket preference via self-report with interventions and comparators having like interfaces and suspension techniques. The meta-analysis of data showed an odds ratio large effect size of $3.77(\mathrm{Cl}=0.34,42.17)$ using a random effects model. Again substantial statistical heterogeneity existed between the studies (Chi square=21.96, $p<0.0001,{ }^{2}=86 \%$ ). There was no difference in preference of socket design ( $p=0.28$ ) when comparing studies using same suspension and interface methods within their study groups. 


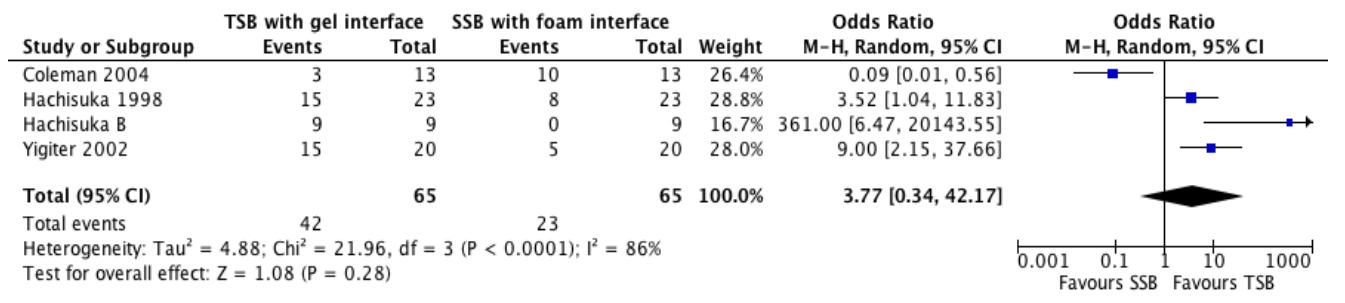

Figure 16: Socket preference (Four experimental studies comparing the TSB with gel interface and distal pin suspension and SSB socket design with foam interface, and proximal suspension method)

There were four studies with a total of 65 participants utilising the TSB design with gel interface and distal suspension and SSB design with foam interface and proximal suspension. The majority of participants (65.6\%) preferred the TSB design with $35.4 \%$ of participants indicating preference for the SSB design, with some participants indicating a preference for both designs. ${ }^{23,48 a, 48 b, 55}$

Two of these studies assessed subjects in their interim phase of rehabilitation, which refers to the first ambulation post-amputation. ${ }^{55,48 b}$ As interim patients, subjects had not previously worn a prosthesis and therefore any influence of preconceived ideas for a particular socket design were unlikely to exist at the time of testing. Hence socket preference would be based only on the experiences that occurred within the study period.

There were 58 participants reporting on socket preference via self-report comparing the TSB with gel interface and distal pin suspension and SSB socket design with foam interface and proximal suspension method. The meta-analysis (Figure 17) shows an odds ratio of huge effect size $34.94(\mathrm{Cl}=1.04,1178.68)$ using a random effects model. TSB socket design was preferred in the rehabilitation population group, with a statistically significant effect size of $(p=0.05)$; however there was substantial heterogeneity as observed by the extremely wide confidence intervals (Chi square $=$ $\left.2.92, p=0.09,1^{2}=66 \%\right)$. 


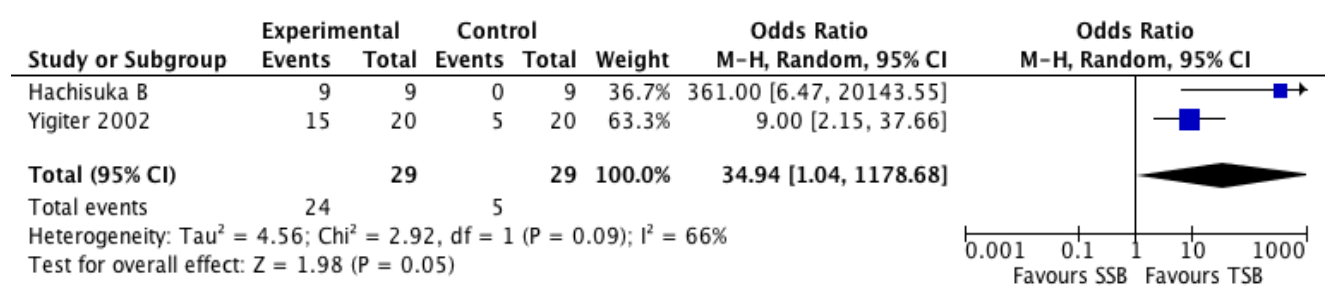

Figure 17: Socket preference (Two experimental studies comparing the TSB with gel interface and distal pin suspension and SSB socket design with foam interface and proximal suspension method in the early rehabilitation phase)

Both studies revealed an overall preference for the TSB over the SSB sockets, with combined results being that $82.7 \%$ of participants preferred the TSB design and $17.3 \%$ of participants preferred the SSB design.

In summary, the included studies revealed there is a reported preference for the TSB socket design. However the preference was not statistically significant except for within the context of early rehabilitation groups. In the latter, a clear preference was found, with subjects 34 times more likely to choose the TSB compared to the SSB design. In terms of implications for practice the findings of the review on the self-selected socket preference are arguably of critical importance as they reveal the patients choice regardless of what the objective measures indicate.

\subsubsection{Frustration}

Three experimental studies $23,50,51$ looked at the results for the PEQ validated sub-scale of Frustration. ${ }^{35}$ In the PEQ, level of frustration was determined by two questions: 'How frequently were you frustrated with your prosthesis?'; 'Rate the level of frustration at the most frustrating time'. A visual analogue scale was used ranging from 0 to $100 \mathrm{~mm}$, with zero representing 'no frustration' and 100 representing the 'most frustrating experience'. The data pooled from these studies is presented in Table 13, with results combined in meta-analysis and shown in Figure 18. 
Table 13: Studies included in the meta-analysis assessing frustration.

\begin{tabular}{|c|c|c|c|c|}
\hline Study ID & Study design & Participants & $\begin{array}{l}\text { Intervention/ } \\
\text { comparator }\end{array}$ & $\begin{array}{l}\text { Frustration } \\
\text { mean (SD) } 0-100\end{array}$ \\
\hline $\begin{array}{l}\text { Coleman } \\
2004^{23}\end{array}$ & \multirow{3}{*}{$\begin{array}{l}\text { Experimental } \\
\mathrm{RCT}\end{array}$} & $\begin{array}{l}\mathrm{n}=13 \\
\text { Aetiology: } V / T / O(0 / 13 / 0)\end{array}$ & $\begin{array}{l}\text { TSB: GI, PS } \\
\text { SSB: FI, SC-S }\end{array}$ & $\begin{array}{l}\text { TSB } 63.2(27.1) \\
\text { SSB } 71.1(34)\end{array}$ \\
\hline $\begin{array}{l}\text { Engsberg } \\
2006^{50}\end{array}$ & & $\begin{array}{l}n=43 \\
\text { Aetiology: V/T/O (unknown) }\end{array}$ & $\begin{array}{l}\text { TSB } \\
\text { SSB }\end{array}$ & $\begin{array}{l}\text { TSB } 73(32) \\
\text { SSB } 79(25)\end{array}$ \\
\hline $\begin{array}{l}\text { Klute } \\
2011^{51}\end{array}$ & & $\begin{array}{l}\mathrm{n}=5 \\
\text { Aetiology: V/T/O (1/4/0) }\end{array}$ & $\begin{array}{l}\text { TSB: GI, VS } \\
\text { SSB: GI, PS }\end{array}$ & $\begin{array}{l}\text { TSB } 43(29) \\
\text { SSB } 91(11)\end{array}$ \\
\hline
\end{tabular}

Abbreviation: TSB: Total surface bearing; SSB: Specific surface bearing; GI: Gel interface; FI: Foam interface; PS: Pin suspension; SC-S: Supracondylar suspension; VS: Vacuum suspension; Aetiology= V: Vascular, T: Traumatic, O: Other; Yrs: Years; SD: Standard deviation

Overall, 122 participants reported on frustration with using their prosthesis, as measured by the PEQ frustration sub-scale. The meta-analysis shows a large effect size (WMD=-18.46; $C l=-41.94,5.01)$ using a random effects model. There was substantial statistical heterogeneity between the studies (Chi square $=7.79, p=0.02$, $1^{2}=74 \%$ ). A higher level of frustration was reportedly experienced with the SSB socket, however this was not statistically significant $(p=0.12)$.

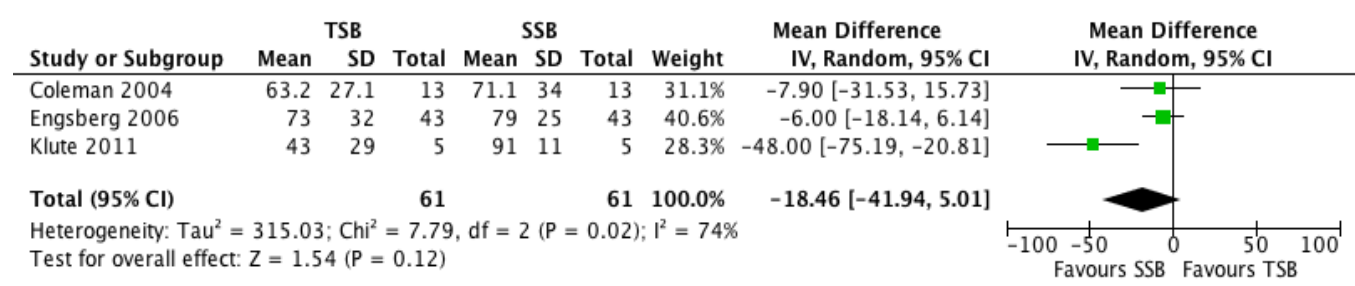

Figure 18: Three experimental studies comparing TSB socket design with SSB socket design in terms of frustration with prosthesis

\subsubsection{Utility}

Two studies ${ }^{23,50}$ looked at the results for the PEQ validated sub-scale of utility. ${ }^{35}$ In the $P E Q$, utility was determined using eight questions regarding socket fit, weight, comfort in standing and sitting, balance, energy, feel of the surface and donning. A visual analogue scale was used from 0 to $100 \mathrm{~mm}$, with zero representing 'terrible' and being the worst possible outcome and 100 representing 'excellent' and being the best 
possible outcome. The data pooled from these studies is presented in Table 14, with results combined in meta-analysis and shown in Figure 19.

Table 14: Studies included in meta-analysis assessing utility

\begin{tabular}{|c|c|c|c|c|}
\hline Study ID & Study design & Participants & $\begin{array}{l}\text { Intervention/ } \\
\text { comparator }\end{array}$ & $\begin{array}{l}\text { Utility } \\
\text { mean (SD) 0-100 }\end{array}$ \\
\hline $\begin{array}{l}\text { Coleman } \\
2004^{23}\end{array}$ & Experimental & $\begin{array}{l}\mathrm{n}=13 \\
\text { Aetiology: V/T/O (0/13/0) }\end{array}$ & $\begin{array}{l}\text { TSB: GI, PS } \\
\text { SSB: FI, SC-S }\end{array}$ & $\begin{array}{l}\text { TSB } 62.8(25.4) \\
\text { SSB } 70.7(23.7)\end{array}$ \\
\hline $\begin{array}{l}\text { Engsberg } \\
2006^{50}\end{array}$ & RUI & $\begin{array}{l}n=43 \\
\text { Aetiology: V/T/O (unknown) }\end{array}$ & $\begin{array}{l}\text { TSB } \\
\text { SSB }\end{array}$ & $\begin{array}{l}\text { TSB } 80(17) \\
\text { SSB } 82(16)\end{array}$ \\
\hline
\end{tabular}

Abbreviation: TSB: Total surface bearing; SSB: Specific surface bearing; Gl: Gel interface; FI: Foam interface; PS: Pin suspension; SC-S: Supracondylar suspension; Aetiology= V: Vascular, T: Traumatic, O: Other; Yrs: Years; SD: Standard deviation

Across the two studies, there were 112 participants who provided data for utility as measured by the PEQ utility sub-scale. The meta-analysis of data showed an effect size of (WMD=-2.71; $\mathrm{Cl}=-9.25,3.84)$ using a random effects model. There was no statistical heterogeneity between the studies (Chi square $=0.33, p=0.57,1^{2}=0 \%$ ). There was no statistically significance difference in reported utility between the two socket designs $(p=0.42)$.

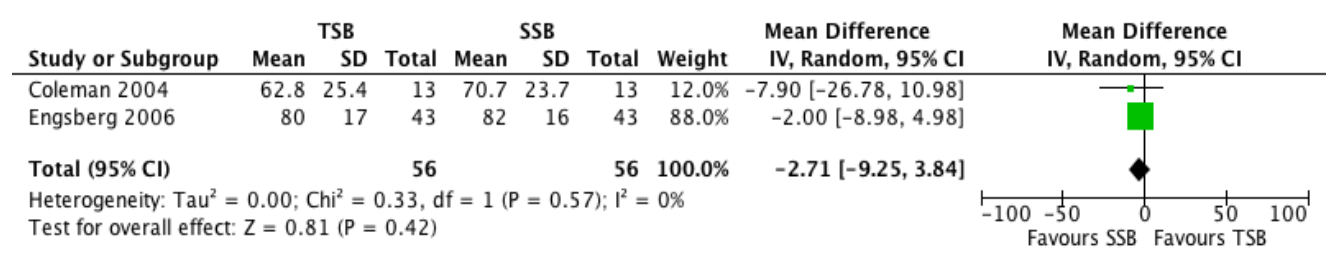

Figure 19: Two experimental studies comparing the TSB socket design with the SSB socket design in terms of utility of the prosthesis

\subsubsection{Well-being}

Two studies ${ }^{23,50}$ examined the results for the PEQ validated sub-scale of well-being. ${ }^{35}$ In the PEQ, well-being was determined through eight questions regarding socket fit, weight, comfort in standing and sitting, balance, energy, feel of the surface and donning. A visual analogue scale was used ranging from 0 to $100 \mathrm{~mm}$, with zero 
representing 'terrible' and being the worst possible outcome and 100 representing 'excellent' and being the best possible outcome. The data pooled from these studies is presented in Table 15, with results combined in meta-analysis and shown in Figure 20.

Table 15: Studies included in the meta-analysis assessing well-being.

\begin{tabular}{|c|c|c|c|c|}
\hline Study ID & Study design & Participants & $\begin{array}{l}\text { Intervention/ } \\
\text { comparator }\end{array}$ & $\begin{array}{l}\text { Well-being } \\
\text { mean (SD) 0-100 }\end{array}$ \\
\hline $\begin{array}{l}\text { Coleman } \\
2004^{23}\end{array}$ & Experimental & $\begin{array}{l}\mathrm{n}=13 \\
\text { Aetiology: V/T/O (0/13/0) }\end{array}$ & $\begin{array}{l}\text { TSB: GI, PS } \\
\text { SSB: FI, SC-S }\end{array}$ & $\begin{array}{l}\text { TSB } 80.5(18.9) \\
\text { SSB } 82.4(16.7)\end{array}$ \\
\hline $\begin{array}{l}\text { Engsberg } \\
2006^{50}\end{array}$ & RCT & $\begin{array}{l}\mathrm{n}=43 \\
\text { Aetiology: V/T/O }\end{array}$ & $\begin{array}{l}\text { TSB } \\
\text { SSB }\end{array}$ & $\begin{array}{l}\text { TSB } 85(20) \\
\text { SSB } 86(20)\end{array}$ \\
\hline
\end{tabular}

Abbreviation: TSB: Total surface bearing; SSB: Specific surface bearing; GI: Gel interface; FI: Foam interface; PS: Pin suspension; SC-S: Supracondylar suspension; Aetiology= V: Vascular, $T$ : Traumatic, O: Other; Yrs: Years; SD: Standard deviation

Overall, there were 112 participants reporting on well-being as measured by the PEQ well-being sub-scale. The meta-analysis shows an effect size of (WMD=-1.25; $\mathrm{Cl}=-8.44$, 5.95) using a random effects model. There was no statistical heterogeneity between the studies (Chi square $=0.01, p=0.91,1^{2}=0 \%$ ). There was no statistically significant difference in self-reported well-being in relation to socket design $(p=0.73)$, indicating participants felt the same way about wearing and utilising their prosthesis irrespective of design and functional performance.

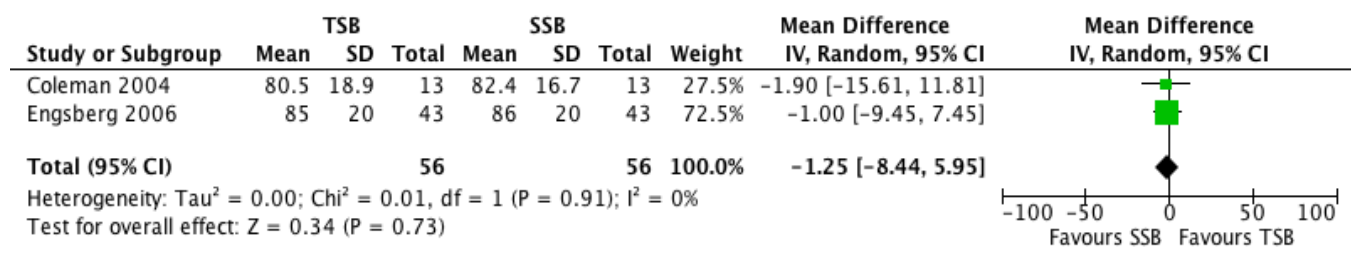

Figure 20: Two experimental studies comparing the TSB socket design with the SSB socket design in terms of self-reported well-being

\subsubsection{Sounds}

The prosthesis as a mechanical device can at times have an audible sound, either from the componentry or due to air expelled from the socket during the stance phase, 
depending on the fit and/or the system used. If sounds are present they can be frustrating to the prosthetic user, often bringing unwanted attention.

Two studies ${ }^{23,50}$ examined the results for the PEQ validated sub-scale sounds. ${ }^{35}$ In the $P E Q$, sounds were determined by two questions regarding the frequency of sound and the extent to which the sound was bothersome. A visual analogue scale was used ranging from 0 to $100 \mathrm{~mm}$, with zero representing 'always' and being the worst possible outcome and 100 representing 'never' and being the best possible outcome. The data pooled from these studies is presented in Table 16, with results combined in metaanalysis and shown in Figure 21.

Table 16: Studies included in meta-analysis assessing sounds

\begin{tabular}{|c|c|c|c|c|}
\hline Study ID & Study design & Participants & $\begin{array}{l}\text { Intervention/ } \\
\text { comparator }\end{array}$ & $\begin{array}{l}\text { Sounds } \\
\text { mean (SD) } 0-100\end{array}$ \\
\hline $\begin{array}{l}\text { Coleman } \\
2004^{23}\end{array}$ & Experimental & $\begin{array}{l}n=13 \\
\text { Aetiology: V/T/O (0/13/0) }\end{array}$ & $\begin{array}{l}\text { TSB: GI, PS } \\
\text { SSB: FI, SC-S }\end{array}$ & $\begin{array}{l}\text { TSB } 63.4(31.6) \\
\text { SSB } 72.8(32.7)\end{array}$ \\
\hline $\begin{array}{l}\text { Engsberg } \\
2006^{50}\end{array}$ & RCT & $\begin{array}{l}\mathrm{n}=43 \\
\text { Aetiology: V/T/O (unknown) }\end{array}$ & $\begin{array}{l}\text { TSB } \\
\text { SSB }\end{array}$ & $\begin{array}{l}\text { TSB } 71(27) \\
\text { SSB } 65(29)\end{array}$ \\
\hline
\end{tabular}

Abbreviation: TSB: Total surface bearing; SSB: Specific surface bearing; GI: Gel interface; FI: Foam interface; PS: Pin suspension; SC-S: Supracondylar suspension; Aetiology= V: Vascular, T: Traumatic, O: Other; Yrs: Years; SD: Standard deviation

Overall, there were 112 participants reporting on sounds of prosthesis as measured by the PEQ sounds sub-scale. The meta-analysis of data showed an effect size of $(\mathrm{WMD}=2.28 ; \mathrm{Cl}=-5.84,15.20)$ using a random effects model. There was some statistical heterogeneity found between the studies (Chi square $=1.21, p=0.27,12=18 \%$ ). There no was statistically significant difference $(p=0.73)$ between the TSB and SSB socket designs in regards to sounds as reported by prosthetic users. 


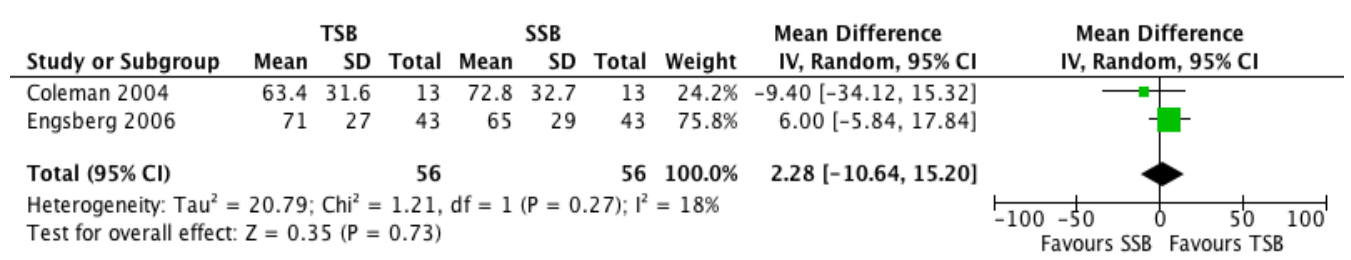

Figure 21: Two experimental studies comparing the TSB socket design with the SSB socket design in terms of the sounds produced by the prosthesis

\subsubsection{Social burden}

Two studies 23,50 looked at the results for the PEQ validated sub-scale of social burden. ${ }^{35}$ Social burden was determined using three questions relating to the burden on the amputee's partner, the hindrance of social activity and care-giving. A visual analogue scale was used ranging from 0 to $100 \mathrm{~mm}$, with zero representing 'extremely burdensome' and 100 representing 'not at all' burdensome. The data pooled from these studies is presented in Table 17, with results combined in meta-analysis and shown in Figure 22.

Table 17: Studies included in meta-analysis assessing social burden

\begin{tabular}{|c|c|c|c|c|}
\hline Study ID & Study design & Participants & $\begin{array}{l}\text { Intervention/ } \\
\text { comparator }\end{array}$ & $\begin{array}{l}\text { Social burden } \\
\text { mean (SD) } 0-100\end{array}$ \\
\hline $\begin{array}{l}\text { Coleman } \\
2004^{23}\end{array}$ & Experimental & $\begin{array}{l}n=13 \\
\text { Aetiology: V/T/O (0/13/0) }\end{array}$ & $\begin{array}{l}\text { TSB: GI, PS } \\
\text { SSB: FI, SC-S }\end{array}$ & $\begin{array}{l}\text { TSB } 82.1(17.2) \\
\text { SSB } 82(24.3)\end{array}$ \\
\hline $\begin{array}{l}\text { Engsberg } \\
2006^{50}\end{array}$ & RCl & $\begin{array}{l}\mathrm{n}=43 \\
\text { Aetiology: V/T/O (unknown) }\end{array}$ & $\begin{array}{l}\text { TSB } \\
\text { SSB }\end{array}$ & $\begin{array}{l}\text { TSB } 92(13) \\
\text { SSB 94(12) }\end{array}$ \\
\hline
\end{tabular}

Abbreviation: TSB: Total surface bearing; SSB: Specific surface bearing; Gl: Gel interface; FI: Foam interface; PS: Pin suspension; SC-S: Supracondylar suspension; Aetiology= V: Vascular, T: Traumatic, O: Other; Yrs: Years; SD: Standard deviation

Overall, there were 112 participants reporting on social burden as measured utilising the PEQ social burden sub-scale. The meta-analysis shows a moderate effect size $(\mathrm{WMD}=-1.80 ; \mathrm{Cl}=-6.82,3.23)$ using a random effects model. There was no statistical heterogeneity found between the studies (Chi square $=0.06, p=0.81, l^{2}=0 \%$ ). There was no statistically significant difference $(p=0.48)$ between the TSB and SSB socket designs 
in regards to social burden; indicating socket design does not have an effect on the level of domestic care-giving from the amputee's perspective.

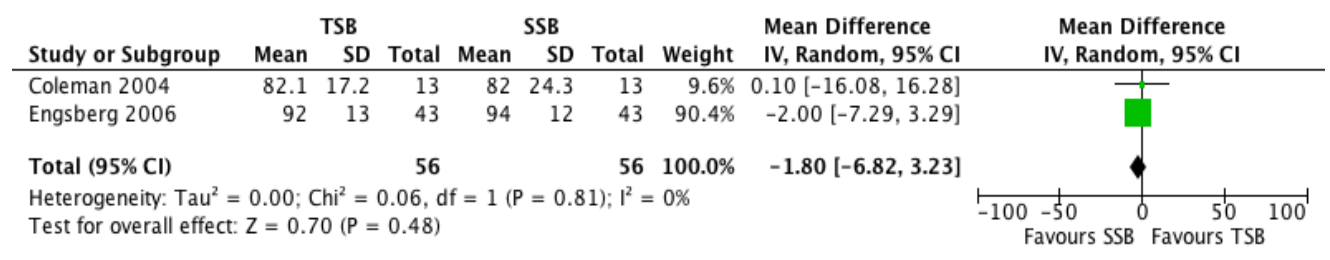

Figure 22: Two experimental studies comparing the TSB and the SSB socket designs in terms of social burden

\subsubsection{Perceived response}

Two studies ${ }^{23,50}$ looked at the results for the PEQ validated sub-scale perceived response. ${ }^{35}$ Perceived response was determined using five questions regarding avoiding strangers' reactions, partner's response to the prosthesis, effects of the prosthesis on the relationship and responses from family members. Visual analogue scales were used ranging from 0 to $100 \mathrm{~mm}$, with 0 representing 'terrible' being the worst possible outcome and 100 representing 'excellent' being the best possible outcome. The data pooled from these studies is presented in Table 18, with results combined in meta-analysis and shown in Figure 23.

Table 18: Studies included in the meta-analysis assessing perceived response

\begin{tabular}{|c|c|c|c|c|}
\hline Study ID & Study design & Participants & $\begin{array}{l}\text { Intervention/ } \\
\text { comparator }\end{array}$ & $\begin{array}{l}\text { Perceived response } \\
\text { mean (SD) } 0-100\end{array}$ \\
\hline $\begin{array}{l}\text { Coleman } \\
2004^{23}\end{array}$ & Experimental & $\begin{array}{l}n=13 \\
\text { Aetiology: V/T/O (0/13/0) }\end{array}$ & $\begin{array}{l}\text { TSB: GI, PS } \\
\text { SSB: FI, SC-S }\end{array}$ & $\begin{array}{l}\text { TSB } 88.7(13.2) \\
\text { SSB } 89.6(13.5)\end{array}$ \\
\hline $\begin{array}{l}\text { Engsberg } \\
2006^{50}\end{array}$ & RCT & $\begin{array}{l}n=43 \\
\text { Aetiology: V/T/O }\end{array}$ & $\begin{array}{l}\text { TSB } \\
\text { SSB }\end{array}$ & $\begin{array}{l}\text { TSB } 91(14) \\
\text { SSB } 98(4)\end{array}$ \\
\hline
\end{tabular}

Abbreviation: TSB: Total surface bearing; SSB: Specific surface bearing; GI: Gel interface; FI: Foam interface; PS: Pin suspension; SC-S: Supracondylar suspension; Aetiology= V: Vascular, $T$ : Traumatic, O: Other; Yrs: Years; SD: Standard deviation

Combining results from the two studies, there were 112 participants reporting on perceived response as measured utilising the PEQ perceived response subscale. The meta-analysis of data showed a large effect size (WMD=-5.79; $C l=-10.56,-1.01)$ using 
a random effects model. There was a low level of heterogeneity between the studies (Chi square $=1.15, p=0.28,12=13 \%)$. A statistically significant $(p=0.02)$ response was identified in favour of the SSB socket, therefore suggesting the SSB socket design was better received than the TSB design.

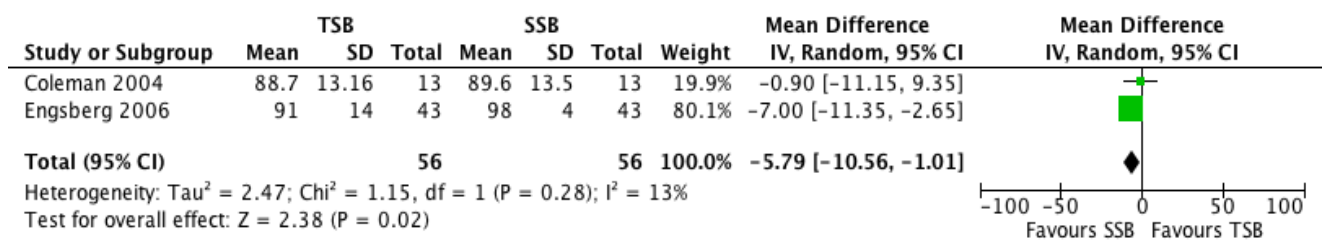

Figure 23: Two experimental studies comparing the TSB and the SSB socket design in terms of perceived response

\subsubsection{Appearance}

Two studies ${ }^{23,50}$ compared the socket design types using the PEQ validated appearance sub-scale. ${ }^{35}$ Appearance was determined using five questions regarding the look of the prosthesis, damage to clothes caused by the prosthesis, damage to the outer cosmetic cover of the prosthesis and the influence of the prosthesis on clothing and shoe choices. Visual analogue scales were used ranging from 0 to $100 \mathrm{~mm}$, with zero representing the 'worst possible' outcome and 100 representing 'not at all' or the best possible outcome. The data pooled from these studies is presented in Table 19, with results combined in a meta-analysis and shown in Figure 24. 
Table 19: Studies included in the meta-analysis assessing appearance

\begin{tabular}{|l|l|l|l|l|}
\hline Study ID & Study design & Participants & $\begin{array}{l}\text { Intervention/ } \\
\text { comparator }\end{array}$ & $\begin{array}{l}\text { Appearance } \\
\text { mean (SD) } \mathbf{0 - 1 0 0}\end{array}$ \\
\hline Coleman 200423 & \multirow{2}{*}{$\begin{array}{l}\text { Experimental } \\
\text { RCT }\end{array}$} & $\begin{array}{l}\mathrm{n}=13 \\
\text { Aetiology: V/T/O (0/13/0) }\end{array}$ & SSB: FI, SC-S & SSB 78.7 (17.4) \\
\cline { 3 - 6 } & $\mathrm{n}=43$ & TSB & TSB 79 (14) \\
& Engsberg 200650 & Aetiology: V/T/O & SSB & SSB 79 (13) \\
\hline
\end{tabular}

Abbreviation: TSB: Total surface bearing; SSB: Specific surface bearing; GI: Gel interface; FI: Foam interface; PS: Pin suspension; SC-S: Supracondylar suspension; Aetiology= V: Vascular, T: Traumatic, O: Other; Yrs: Years; SD: Standard deviation

Overall, there were 112 participants reporting on appearance of the prosthesis as measured by the PEQ sub-scale. The meta-analysis of data showed a weak effect size $(W M D=1.08 ; C l=-4.09,6.25)$ using a random effects model. There was no statistical heterogeneity between the studies (Chi square $=0.76, p=0.38,1^{2}=0 \%$ ). There was no statistically significant difference $(p=0.68)$ between participants' responses regarding the appearance of the two different prosthetic socket designs.

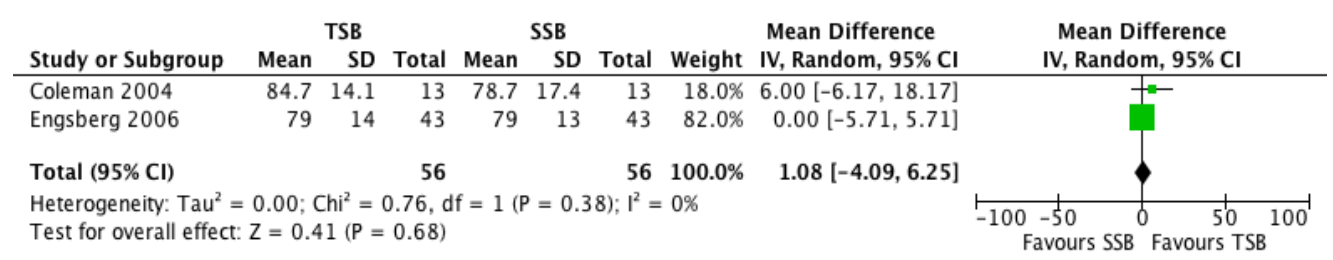

Figure 24: Two experimental studies comparing the TSB and SSB socket designs in terms of their appearance

\subsubsection{Satisfaction}

Selles et al. assessed satisfaction through the use of the Dutch version of the PEQ. ${ }^{52}$ Satisfaction comprises one of the 15 sub-scales of the PEQ, however it has not been validated. The author reported higher satisfaction among the TSB group $(M=148.9$, $S D=58.0)$ at baseline and follow-up $(M=147.0, S D=48.4)$ compared to the SSB group $(M=135.1, S D=48.2)$ at baseline and follow-up $(M=150.5, S D=49.1)$. These results show no difference in satisfaction between the two socket designs. 


\subsubsection{Energy usage}

One of the goals of amputee rehabilitation is the efficient use of a prosthesis. This can be measured by assessing the energy cost or energy expenditure, whereby oxygen $\left(\mathrm{O}_{2}\right)$ cost is recorded in relation to walking speed. Waters and Mulroy describe energy expenditure, or the power requirement (rate of $\mathrm{O}_{2}$ consumption) as the millilitres of $\mathrm{O}_{2}$ consumed per kilogram of body weight per minute ( $\mathrm{m} / \mathrm{kg}$ per min). ${ }^{53}$ The $\mathrm{O}_{2}$ cost is determined by dividing the power requirement (rate of $\mathrm{O}_{2}$ consumption) by the speed of walking. ${ }^{53} \mathrm{It}$ has been reported that individuals with trans-tibial amputations have a $20 \%$ higher rate of $\mathrm{O}_{2}$ uptake that parallels the normal energy-speed relationship. ${ }^{54}$

Engsberg et al. is the only study in this review that reported on energy expenditure comparing the SSB and TSB socket designs. ${ }^{50}$ This study includes a sample of 43 independent ambulators without significant health problems, with a mature residuum and without cause of amputation reported. Testing of energy expenditure was done after a period of four weeks acclimatisation with each socket design, assessed by $\mathrm{O}_{2}$ uptake during a graded exercise test (GXT). Thirty-six subjects were included in the GXT, as health status was determined as not putting them at risk whilst performing the test. Oxygen uptake, pulmonary ventilation, heart rate, blood pressure and rate of perceived exertion were monitored during each of the four stages of the GXT. Results were based on the measurements taken during the final 30 seconds of the last stage of the GXT.

The authors reported that energy expenditure was not different in subjects comparing the TSB socket design $\left(M=13.2, S D=2.6 \mathrm{ml} /\left(\mathrm{kg}^{*} \mathrm{~min}\right)\right)$ and the SSB socket design $\left(M=13.2, S D=2.3 \mathrm{ml} /\left(\mathrm{kg}^{*} \mathrm{~min}\right)\right)$; indicating that socket design change does not have an effect on oxygen uptake.

\subsection{Results summary}

To summarise the findings of the review on the question of the comparative effectiveness of the TSB and SSB socket design type the review identified and analysed 28 different outcome measures used to assess particular health domains across 11 included studies, with results detailed in the table of findings (Appendix VIII). Of the 28 measures, when comparing the TSB to the SSB socket design only, 21 measures found no difference between the socket designs. Four measures found 
significant differences in favour of the TSB design and three measures found significant differences in favour of the SSB design. The measures that showed a significant preference for the TSB prosthesis were: step width, balance, weight bearing and activities of daily living. Those that showed a significant difference in favour of the SSB socket design type were: step count, wear time and perceived response.

Sub-group analyses were conducted for six measures to explore how differences in interface may affect the direction and size of measures of effect. Four of these measures, socket comfort, socket preference, frustration experience and utility of prosthesis, identified no difference in terms of the two socket designs. For two measures, walk speed and cadence, the analysis found a significant difference in favour of the TSB design.

Further analysis with gait speed and socket preference outcome measures, catering for gel interface and distal pin suspension in the TSB design, compared to foam interface and supra-condylar suspension in the SSB design; identified gait speed favoured the TSB design with statistical significance and patient preference for TSB design without statistical significance.

To summarise the results for the range of outcomes for which measures were analysed, the main findings were as follows:

i) The studies present mixed findings concerning which socket design is most effective across a wide spectrum of outcomes and measures.

ii) Interpreting the evidence is difficult as what is compared in the studies is different, with interface gel liners used typically with the TSB design and foam interface with the SSB design. Likewise suspension mechanism comprises mostly distal attachment or negative pressure in the TSB design and proximal supracondylar attachment in the SSB design.

iii) There is evidence to suggest that the TSB socket design is an appropriate prescription choice and may be a preferred fit in regards to comfort and patient preference in the inpatient rehabilitation setting. 


\section{Chapter 4 Discussion}

The final chapter of this dissertation has two objectives. The first is to provide an overview of the main findings of the review and highlight particularly noteworthy aspects of the included studies and the synthesis (section 4.1). The second objective is to discuss the limitations of the review (section 4.2).

\subsection{Main findings}

This systematic review included 11 studies; 10 of which are level 2 primary studies of effectiveness and one a level 3 study of effectiveness based on the JBI Levels of Evidence classification system (Appendix IX). The review compared the TSB and SSB prosthesis socket design using outcome measures relating to four key domains of interest: function and mobility, comfort and pain, quality of life and energy expenditure. The included studies provided a relatively large amount of data, and range of measures to synthesise in order to address the question of the relative effects of the two socket design types.

The synthesis, which included a number of meta-analyses, found that the existing evidence base presents mixed findings on the relative superiority of the socket designs. Differences in the interventions/socket design types used across the studies - in particular the nature of the interface used in the TSB and SSB sockets - emerged as a potential confounder that may have affected the size and direction of effect measures.

Although no clear preference for either socket design was identified when comparing the two designs, sub-group analysis revealed better performance with the TSB socket when using a gel or silicon interface compared to a foam interface. Preference for distal pin suspension compared to proximal supracondylar suspension was identified in subgroup analysis that considered walking speed and cadence measures.

Regarding quality of life, pooled data indicated a preference for the TSB design. Subgroup analysis was stronger for the TSB design when using the gel interface and distal suspension compared to the foam interface and proximal suspension. These results are suggestive that the gel interface with distal pin suspension method may be a preferred choice for the individual with amputation. 
When considering studies that recruited participants from the rehabilitation setting, the TSB design was a clear choice. This is clinically revealing, as the TSB design is the most commonly prescribed prosthesis for patients after they have completed rehabilitation and once the residuum has stabilised in volume.

Another finding worth highlighting is that the reported preference measures showed a preference for the TSB socket design. This preference, which is arguably critical from a clinical perspective was not however statistically significant except for within the context of early rehabilitation groups.

\subsubsection{Nomenclature}

The definitions of TSB and SSB have been described thoroughly; however they may be different to other research describing these socket designs. Differences in terminology were apparent across the included studies; therefore the authors were contacted for clarification where required. If authors were unable to be contacted, assumptions were made based on the description of the methodology of studies to determine similarities in socket design to meet the inclusion criteria.

Without universal standardised nomenclature or terminology, differing terminologies can limit interpretation for future investigators. With studies originating in Asia, Europe and North America, the results of the review in this thesis confirm the similar use of terminology and understanding of socket design descriptions to be consistent across all geographical areas represented.

Consistency in using specific domains when assessing a client's needs was identified and studies used similar measures to report on outcomes relating to the identified domains. It is reasonable to conclude that whilst prosthetic design and manufacture technique can be varied; the use of universal terminology and training techniques needs to be maintained to assist clinical practice and research.

\subsubsection{Variations in interventions and comparators}

Despite the base terminology reported with consistency, inconsistencies were identified in elements of the prosthetic designs utilised, which were considered as a limitation of this review. Overall, there were nine combinations of design, with differences in the type of interface and suspension utilised. Even within the grouped analysis of gel interface, there were four variations of gel interface liners, all with different properties that may 
have had some influence on the measured outcomes. Likewise, the review included prostheses with a variety of suspension methods. Three different suspension methods were reported in the TSB design and three in the SSB design.

The variation in prosthesis interface and suspension identified is typical of the presentation in clinical practice and not necessarily linked to practitioner, facility, region, country or funding provision. Prescription choices are led by clinical assessment of the residuum, with interface selected depending on residuum length, skin condition, amount of soft tissue to be controlled, client's tolerances to distal suspension and knee joint restriction of movement and type and frequency of the activities performed as part of the patient's daily life.

\subsubsection{Variation in study objectives/intention}

An additional noteworthy feature was the high level of variation in the primary intention or objectives of the included studies and the outcomes they examined. Whilst seven studies shared the primary aim of comparing the differences in and effectiveness of trans-tibial socket design;24,48a,48b,52-55 three studies set out to investigate differences in interface use, ${ }^{23,54,56}$ and one study investigated the difference in suspension method. ${ }^{51}$ However, by doing so, the latter four studies used the TSB and SSB socket designs and hence were included in this review.

\subsubsection{Comfort and pain}

Surprisingly, the evidence revealed no difference between the TSB and SSB socket designs within the domain of comfort and pain, using six self-report measures across seven studies. Clinically, practitioners would expect an improvement in residual limb health with the TSB design due to reduced specific loading and an even distribution of forces across the soft tissue of the residuum. Coupled with the gel interface, the TSB design has been a prescription option often undertaken for addressing specific residuum pain.

Findings indicate that when participants reported presence of pain, either neurological or phantom pain, a change of socket design did not influence, alleviate or heighten the pain. The studies providing the results were cross-over design, parallel group design and cohort studies. There was no combination of socket design in any of the studies that led to a difference or influence on pre-existing pain or discomfort. 
These findings are of clinical significance as one of the contributing factors in the prescription clinic setting to replace the socket with a new design and/or interface is the presence of self-reported pain. These results encourage practitioners to investigate the underlying cause of pain with rigor and to investigate treatments to address pain with other measures, which may or may not be in conjunction with a new prosthetic prescription.

\subsubsection{Community based activity}

Other results of interest that were unexpected involved the community-based measure of functional activity, recorded with a step activity monitor. Coleman et al. reported a significantly higher wear time and steps taken per day with the traditional foam interface and supracondylar suspension SSB socket compared to the gel interface distal pin suspension TSB design. ${ }^{23}$ These results were in contrast to the laboratory-based walking measures. Caution must be taken when interpreting these results due to the provision for participants in this study to utilise their pre-existing prosthesis during the study duration, which may have impacted on the results. Though it is reasonable to surmise that the reduction in wear time is likely due to the participant's preference for their previous design, which could be influenced by the type of gel interface utilised in this study.

\subsubsection{Quality of Life}

The most commonly reported measure was the client-reported socket preference, which indicates all studies across the three continents were mindful of client satisfaction and individual preference in determining the prosthesis prescription. Quality of life is difficult to measure and can be overlooked by funding bodies as a contributing reason for prescription. To support movement towards patient-centred prescription choices, clinicians and prescribers should continue to marry the evidence-based information and rationale for prescription with the client's preference for prosthetic fit, comfort and usability.

Involving the client in the prescription process is paramount for clinicians, prescribers and researchers, as is the range of measures used to assess the client's needs. 
The variation in the findings was not unexpected and is typical of prescription and fitting in clinical practice. Therefore, it was important to consider the influence of the TSB and SSB designs on participation outcomes and to group together like studies in relation to use of interface and suspension technique, or rehabilitation setting and undertake subgroup analysis in an effort to determine the influence of interface or suspension on outcomes for persons with amputation.

After conducting a meta-analysis and considering the results of individual studies, it was found that the TSB socket design for trans-tibial amputation is as effective as the traditional SSB design in terms of health outcomes and allowing for life participation. The combination of the gel interface and a more secure suspension system is likely to have contributed to these findings.

\subsection{Limitations of the review}

The first limitation of this review to note relates to its scope; only studies published in the English language were sought and considered for inclusion. There may be additional non-English language studies that may have contributed useful data which may, if they had been considered, led to different conclusions being drawn.

The methodological weaknesses in the design of included studies, relating to selection, performance and attrition and detection bias, summarised in Table 1, are a second feature of the review to note as a limitation. To summarise these shortcomings, which may have introduced a degree of error in the measures of effects, only five studies included randomisation in the study design with the implication that factors other than the difference in socket design type may have influenced the size and direction of the measurement of effects. The nature of the interventions prevents successful blinding of the clients to the intervention used, and concealment of socket design is difficult when collecting functional outcome data, thereby investigators were not blinded to interventions.

A third limitation to note is that most of the measures of effect did not control adequately for the potential effect of the interface liner and mode of suspension surrounding the socket on the outcome measures. Only one study and five measures included in the meta-analysis were robust in the sense that they were able to isolate the socket difference effect from a potential effect of the interface/liner and socket suspension. 
A fourth limitation is that whereas the review was able to identify and include in the synthesis/analysis a relatively wide range of well recognised measures of effects, from a relatively large number of studies and conduct a good number of meta-analyses - a strength of the review - the number of studies and participants in each meta-analysis was small.

A fifth limitation is that the populations in the studies included in the review were heterogeneous with respect to age, cause of amputation and length of time from amputation and the analysis was not able to generate measures by age, aetiology/cause of amputation or any other key characteristics in the synthesis.

A sixth limitation is that in three of the included studies, there was a small degree of doubt about the nature of the socket design (TSB or SSB) or the type of interface utilised. The authors of the studies could not indicate/describe and hence the reviewers made a classification based on their expert opinion.

A final limitation to note is that the measure of effects determined and the conclusions drawn are based on studies that measured effects over varied periods of time/follow up. Currently there are no widely available evidence-based guidelines for acclimatisation of socket design in a research context, however clinical practitioners would allow approximately three weeks for clients to adapt to a new socket intervention prior to review of outcomes. 


\section{Chapter 5 Conclusions and recommendations for practice and research}

This final chapter's objective is to conclude and draw inferences from the evidence review for research and practice.

\subsection{Conclusions}

A substantial body of evidence exists on the effectiveness of the TSB compared to the SSB prosthesis socket design for adolescents and adults with a trans-tibial amputation. This evidence has examined the utility of the TSB compared to the SSB prosthesis socket design for function and mobility, comfort and pain, quality of life and energy expenditure. The evidence suggests that irrespective of the interface or suspension method, there is little difference between the socket designs except for step width, balance, weight bearing and ADL physical measures.

Variation in the interface liners and in the modes of suspension across the studies that have compared the two socket designs complicates understanding of the relative effects of the two socket designs on the outcomes of function and mobility, comfort and pain, quality of life and energy expenditure. It is therefore not possible from the evidence to determine whether interface alone makes a difference to socket design effect and it cannot be concluded whether it is the combination of the interface and socket design that leads to better outcomes, or the interface and/or suspension that leads to better outcomes.

Whilst there are ten good quality level 2 primary studies 23,24,48a,48b,50-55 (Appendix IX) in the evidence base, that were included in this review, there are no level 1 studies. To determine with a high degree of certainty whether there is any significant difference in the effect of trans-tibial socket designs; level 1 primary studies of RCT design utilising the same interface and suspension methods for experimental and control groups and conducted across multiple sites and regions are required.

As this review showed, there are many different ways to judge and measure the relative worth of the one socket design type compared to the other. Hence, to provide evidence to inform practice, the studies need to measure a broad spectrum of outcomes. 
Whilst physical-based measures of participation mainly relating to function and mobility are objective and can quantify improvements in research terms, it is often the selfreported subjective results relating to comfort and quality of life that hold greater meaning for the prosthetic user. For example, the ability to walk with increased speed and symmetrical step pattern over a distance of 10 meters indoors would not be as meaningful to the person with amputation as being able to independently don and doff their prosthesis and participate in a range of daily activities in relative comfort. Community-based objective and subjective measures provide clinicians and researchers with the relevant context to the prosthetic provision and therefore a patientcantered approach to prescription is recommended.

Given that socket prescription needs to take into account residual limb tolerances and funding availability for interface materials, not all clinics have the option to provide a choice of socket design. However, if that choice is available it is appropriate for the practitioner to know that the TSB socket design, if prescribed as per clinical indications may be as effective for trans-tibial amputees as the SSB design has been shown to be over many decades.

This review suggests that prosthetic users should be given the option to try the TSB socket design where clinically indicated and cost-supported to do so. To this end, clinician skill-set needs to incorporate new techniques and technologies within their scope of practice and undergraduate prosthetic students should be required to complete training for provision of the gel interface TSB socket design in preparation for clinical practice. This may require investment in building necessary training modules for practicing clinicians, as it is unknown which tertiary institutions delivering prosthetic training undertake TSB socket design education, or how many clinical facilities and what percentage of clinicians practice using this technique.

With funding bodies, both private and public, looking to extend the health dollar further, longitudinal studies are required to assess the long-term residual limb health, longevity of prosthetic provision and correlate with participation in community activities to assess the ongoing effects of trans-tibial prosthetic prescription with respect to socket design, interface and suspension methods, and componentry prescription. 


\subsection{Recommendations for practice}

The finding that the TSB socket design within prostheses for people with trans-tibial amputation may be as effective as the SSB socket design suggests that prescription in clinical practice should be guided by prescriber knowledge, clinician skill-set for manufacture, patient preference and funding availability. (Level 2)

The review highlights the need for investment to ensure prosthetists have the skill-set necessary to deliver the TSB socket technique if prescribed, or indicated as a client preference. (Level 3)

Given that the TSB prescription goes hand-in-hand with gel interface provision, the review suggests that funding bodies should consider changing the paradigm of care, with provisions made for maintaining limb health and enhancing quality of life through the use of the gel interface and TSB socket design combination. (Level 3)

\subsection{Recommendations for research}

This review raises the following priorities for future research:

First, primary studies and synthesis on the cost effectiveness of the alternative socket designs. Building the evidence base on how costs compare for the different socket designs is critical especially because the existing evidence base suggests there is little difference with respect to health effects for people with amputations. The cost effectiveness research should adopt a societal perspective and thereby help provide decision makers with a knowledge base that can be used to improve the efficiency of scarce societal resources.

A second research priority identified by the review is additional high quality studies, including qualitative and mixed method design, to further evaluate patient experiences with socket design. Studies on the effects of the different socket design types that include a longer time frame in outcome measurement, include larger participant groups, conduct sub-group analysis for key characteristics of participants (e.g. age, gender, aetiology, years since amputation) and which are careful to control for potential confounders such as interface and suspension type are required. 


\section{Conflict of interest}

There are no conflicts of interest to declare by the author. 


\section{References}

1. Kapp S, Miller JA. Lower Limb Prosthetics. In: Pasquina PF, Cooper RA, editors. Care of the combat amputee (textbook of military medicine). Dept. of the Army; 2010.p 553-80.

2. Sansam K, Neumann V, O'Connor R, Bhakta B. Predicting walking ability following lower limb amputation: a systematic review of the literature. Journal of rehabilitation medicine. 2009;41:593-603.

3. Day HJB. A review of the consensus conference on appropiate prosthetic technology in developing countries. Prosthetics and orthotics international. 1996;20:15-23

4. Staats TB. The rehabilitation of the amputee in the developing world: a review of the literature. Prosthetics and orthotics international. 1996;20(1):45-50.

5. Gailey RS, Roach KE, Applegate EB, Cho B, Cunniffe B, Licht S, Maguire M, Nash MS. The amputee mobility predictor: an instrument to assess determinants of the lower limb amputee's ability to ambulate. Archives of physical medicine and rehabilitation. 2002;83:613-27.

6. Board WJ, Street GM, Caspers C. A comparison of trans-tibial amputee suction and vaccuum socket conditions. Prosthetics and orthotics international. 2001;25:202-209.

7. Kristinsson 0 . The ICEROSS concept: a discussion of a philosophy. Prosthetics and orthotics international. 1993;17:49-55.

8. SA Health, Statewide rehabilitation clinical network. Model of amputee rehabilitation in South Australia.

http://www.sahealth.sa.gov.au/wps/wcm/connect/

9. Eldar $\mathrm{R}$, Jelic $\mathrm{M}$. The association of rehabilitation and war. Disability and 
rehabilitation. 2003;25(18):1019-1023.

10. Amputee coalition of America http://www.amputee.coalition.org/inmotion/novdec-07/history-prosthetics.html

11. Australian Orthotic and Prosthetic Association.

http://www.aopa.org.au/documents/item/36

12. Esquenazi A. Amputation rehabilitation and prosthetic restoration. From surgery to community reintegration. Disability and rehabilitation. 2004;26(14/15):831-6.

13. van Velzen J. Physical capacity and walking ability after lower limb amputation: a systematic review. Clinical rehabilitation. 2006;20:999-1016.

14. Isakov $E$, Burger $H$, Gregoric $M$, Marincek $C$. Stump length as related to atrophy and strength of the thigh muscles in trans-tibial amputees. Prosthetics and orthotics international. 1996;20:96-100.

15. Van der Linde H, Hofstad CJ, Geurts ACH, Postema K, Geertzen JHB, van Limbeek J. A systematic literature review of the effect of different prosthetic components on human functioning with a lower-limb prosthesis. Journal of rehabilitation research and development. 2004;41(4):555-70.

16. Klute GK, Berge JS, Orendurff MS, Williams RM, Czerniecki JM. Prosthetic intervention effects on activity of lower-extremity amputees. Archives of physical medicine and rehabilitation. 2006;87(7)17-22.

17. Condie E, Scott H, Treweek S. Lower limb prosthetic outcome measures: a review of the literature 1995 to 2005. Journal of prosthetics and orthotics. 2006;18(1S):13-45.

18. Penn-Barwell JG. Outcomes in lower limb amputation following trauma: a 
systematic review and meta-analysis. Injury. 2011;42:1474-9.

19. Samuelsson KAM, Töytäri O, Salminen A-L, Brandt $\AA$. Effects of lower limb prosthesis on activity, participation, and quality of life: a systematic review. Prosthetics and orthotics international. Epub February 3, 2012. Available from http://poi.sagepub.com/content/early/2012/02/01/0309364611432794

20. Staats TB, Lundt J. The UCLA total surface bearing suction below-knee prosthesis. Clinical prosthetics and orthotics. 1987;11(3):118-30.

21. Radcliffe CW, Foort J, University of California. Biomechanics Laboratory, San Francisco. The patellar-tendon-bearing below-knee prosthesis. Rev. Edition: Berkeley: Biomechanics Laboratory, Dept. of Engineering, University of California, 1961. Available from: http://trove.nla.gov.au/version/8559906

22. Fergason J, Smith DG. Socket considerations for the patient with a trans-tibial amputation. Clinical orthopaedics and related research. 1999(361):76-84.

23. Coleman KL, Boone DA, Laing LS, Mathews DE, Smith DG. Quantification of prosthetic outcomes: Elastomeric gel liner with locking pin suspension versus polyethylene foam liner with neoprene sleeve suspension. Journal of rehabilitation research and development. 2004;41(4):591-602.

24. Datta D, Harris I, Heller B, Howitt J, Martin R. Gait, cost and time implications for changing from PTB to ICEX® sockets. Prosthetics and orthotics international. 2004;28(2):115-20.

25. Taylor L, Cavenett S, Stepien J, Crotty M. Removable rigid dressings: a retrospective case-note audit to determine the validity of post-amputation application. Prosthetics and orthotics international. 2008;32(2),223-230.

26. Baars EC, Geertzen JH. Literature review of the possible advantages of silicone liner socket use in trans- tibial prostheses. Prosthetics and orthotics international. 2005;(1):27-37. Available from: 
http://poi.sagepub.com/content/29/1/27

27. Kahle JT. Conventional and hydrostatic trans-tibial interface comparison. Journal of prosthetics and orthotics. 1999;11(4):85-91.

28. Government of Western Australia. Department of Health. Western Australian limb service for amputees (WALSA) http://www.health.wa.gov.au/wals/faqs/\#8

29. Queensland Government; Queensland Health; Queensland amputee limb service (QALS)

http://www.health.qld.gov.au/qals/docs/procedure/42089.pdf

30. New South Wales Government; Healthshare; Enable NSW; prosthetic limb service (PLS) Prescription and provision guidelines. http://www.enable.health.nsw.gov.au/-documents/pls-documents/prostheticlimb-service-funding-_sep13.pdf

31. World Health Organisation. The relationship between prosthetic and orthotics services and community based rehabilitation (CBR). A joint ISPO/WHO statement, November 2003 http://www.who.int/disabilities/technology/po_services_cbr.pdf

32. Gholizadeh H, Osman NA, Esraghi A, Ali S, Razak NA. Transtibial prosthesis suspension systems: Systematic review of literature. Clinical biomechanics. 2013 http://dx.doi.org/10.1016/j.clinbiomech.2013.10.01323.

33. World Health Organisation. International classification of functioning disability and health. Available from: http://www.who.int/classifications/icf/en/index.html.

34. Coleman KL, Smith DG, Boone DA, Joseph AW, Del Aguila MA. Step activity monitor: long-term, continuous recording of ambulatory function. Journal of rehabilitation research and development. 1999;36(1):8-18.

35. Legro MW, Reiber GD, Smith DG, Del Aguila M, Larsen J, Boone D. Prosthesis Evaluation Questionnaire for persons with lower limb amputations: assessing prosthesis-related quality of life. Archives of physical medicine and rehabilitation. 1998;79:931-8. 
36. Hanspal RS, Fisher K, Nieveen R. Prosthetic socket fit comfort score. Disability and rehabilitation. 2003;25(22):1278-80.

37. Higgins JPT, Green S (editors). Cochrane Handbook for Systematic Reviews of Interventions Version 5.1.0 [updated March 2011]. The Cochrane

Collaboration, 2011. Available from www.cochrane-handbook.org.

38. Sinha R, and Van den Heuvel W. A systematic literature review of quality of life in lower limb amputees. Disability and rehabilitation. 2011;33(11):883-899.

39. Grant MJ, Booth G. A typology of reviews: an analysis of 14 review types and associated methodologies. Health information and libraries journal. 2009;26:91108.

40. Armstrong R, Hall BJ, Doyle J, Waters E. Cochrane update: 'Scoping the scope' of a cochrane review. Journal of public health. 2011;33(1):147-150

41. Institute TJB. Joanna Briggs Institute Reviewers' Manual: 2011 edition: The Joanna Briggs Institute 2011.

42. Cavenett S, Ko Ko Aung E, White S, Streak J. The effectiveness of total surface bearing compared to specific surface bearing prosthetic socket design on health outcomes of adults with a trans-tibial amputation: A systematic review protocol. The JBI database of systematic reviews and implementation reports. 2012;10(56) Suppl.

43. Smith M, Glass G, Miller T. The benefits of psychotherapy. Baltimore, MD: Johns Hopkins University Press. 1980

44. Sackett DL, Rosenberg WMC, Gray JAM, Haynes RB, Richardson WS. Evidence based medicine: what it is and what it isn't: it's about integrating individual clinical expertise and the best external evidence. BMJ. 1996;312:712.

45. Pearson A, Wiechula R, Court A, Lockwood C. The JBI model of evidencebased healthcare. International journal of evidence based healthcare. 2005;3:207-215.

46. Cleeland CS, Ryan KM. Pain assessment: global use of the brief pain inventory. Annals of the academy of medicine Singapore. 1994;23(2):129-38.

47. The Cochrane Collaboration. Review Manager (RevMan) [Computer program]. 
Version 5.2. Copenhagen: The Nordic Cochrane Centre, 2008. Available from: http://ims.cochrane.org/revman/download

48. Hachisuka K, Dozono K, Ogata H, Ohmine S, Shitama H, Shinkoda K. Total surface bearing below-knee prosthesis: advantages, disadvantages, and clinical implications. Archives of physical medicine and rehabilitation. 1998;79(7):783-9.

49. Goh JCH, Lee PVS, Chong SY. Comparative study between patellar-tendonbearing and pressure cast prosthetic sockets. Journal of rehabilitation research and development. 2004;41(3B):491-502.

50. Engsberg JR, Sprouse SW, Uhrich ML, Ziegler BR, Luitjohan FD. Comparison of rectified and unrectified sockets for transtibial amputees. Journal of prosthetics and orthotics. 2006;18(1):1-7.

51. Klute GK, Berge JS, Biggs W, Pongnumkul S, Popovic Z, Curless B. Vacuumassisted socket suspension compared with pin suspension for lower extremity amputees: effect on fit, activity, and limb volume. Archives of physical medicine and rehabilitation. 2011;92(10):1570-5.

52. Selles RW, Janssens PJ, Jongenengel CD, Bussmann JB. A randomised controlled trial comparing functional outcome and cost efficiency of a total surface-bearing socket versus a conventional patellar tendon-bearing socket in trans-tibial amputees. Archives of physical medicine and rehabilitation. 2005;86(1):154-61.

53. Manucharian SR. An investigation of comfort level trend differences between the hands-on patellar tendon bearing and hands-off hydrocast trans-tibial prosthetic sockets. Journal of prosthetics and orthotics. 2011;23(3):124.

54. Astrom I, Stenstrom A. Effect on gait and socket comfort in unilateral trans-tibial amputees after exchange to a polyurethane concept. Prosthetics and orthotics international. 2004;28(1):28-36.

55. Yigiter K, Sener G, Bayar K. Comparison of the effects of patellar tendon bearing and total surface bearing sockets on prosthetic fitting and rehabilitation. Prosthetics and orthotics international. 2002;26(3):206-12.

56. Datta D, Vaidya SK, Howitt J, Gopalan L. Outcome of fitting an ICEROSS prosthesis: views of trans-tibial amputees. Prosthetics and orthotics international. 1996;20(2):111-5.

57. Health care financing administration (HCFA) common procedure coding system 
(HCPCS). Washington (DC)2001. Available from:

http://ushik.ahrq.gov/ViewltemDetails?system=mdr\&itemKey=65353000

58. Rietman JS, Postema K, Geertzen JHB. Gait analysis in prosthetics: Opinions, ideas and conclusions. Prosthetics and orthotics international. 2002;26:50-7.

59. Nolan L, Wit A, Dudziñski K, Lees A, Lake M, Wychowañski M. Adjustments in gait symmetry with walking speed in trans-femoral and trans-tibial amputees. Gait and posture. 2003;17:142-51.

60. Silver-Thorn MB, Steege JW, Childress DS. A review of prosthetic interface stress investigations. Journal of rehabilitation research and development. 1996;33:253-66.

61. Herzog W, Nigg BM, Read LJ, Olsson E. Asymmetries in ground reaction force patterns in normal human gait. Medicine and science in sports and exercise. 1989;21(1):110-4.

62. Mancini M, Horak FB. The relevance of clinical balance assessment tools to differentiate balance deficits. European journal of physical and rehabilitation medicine. 2010;46(2):239-48.

63. Gruendel TM. Relationships in weight bearing characteristics in standing and ambulatory independance in hemiplegics. Physiotherapy Canada. 1992;44(4):16-7.

64. Bussmann JBJ, Martens WLJ, Tulen JHM, Schasfoort FC, Van Den BergEmons HJG, Stam HJ. Measuring daily behaviour using ambulatory accelerometry: the activity monitor. Behaviour research methods, instruments, and computers. 2001;33(3):349-56.

65. Atkinson TM, Rosenfeld BD, Sit L, Mendoza TR, Fruscione M, Lavene D, Shaw M, Li Y, Hay J, Cleeland C, Scher H, Breitbart W, Basch E. Using confirmatory factor analysis to evaluate construct validity of the brief pain inventory (BPI). Journal of pain and symptom management. 2011;41(3):558-65.

66. Waters RL, Mulroy S. The energy expenditure of normal and pathologocal gait. Gait and posture. 1999;9:207-31.

67. Molen NH. Energy/speed relation of below-knee amputees walking on motordriven treadmill. Internationale Zeitschrift für angewandte Physiologie, einschliesslich Arbeitsphysiologie 1973;31:173-185. Cited in: Unbound Medline: PubMed ID4696826 


\section{Appendices}

\section{Appendix I: Published Systematic Review Protocol}

\section{Review title}

The effectiveness of total surface bearing compared to specific surface bearing prosthetic socket design on health outcomes of adults with a trans-tibial amputation: A systematic review

\section{Reviewers}

Sally Cavenett BPO1,2

Edward Ko Ko Aung MBBS2

Dr Sarahlouise White 3

Dr Judith Streak ${ }^{3}$

${ }^{1}$ Orthotics and Prosthetics South Australia, Repatriation General Hospital, SA Health, Daw Park, SA 5041, Australia and Masters of Clinical Sciences, The Joanna Briggs Institute, Faculty of Health Sciences, The University of Adelaide, SA 5005, Australia.

2Masters of Clinical Science Candidate, The Joanna Briggs Institute, Faculty of Health Sciences, The University of Adelaide, SA 5005, Australia

${ }^{3}$ Research Fellow, The Joanna Briggs Institute, Faculty of Health Sciences, The University of Adelaide, SA 5005, Australia

\section{Review objective}

The objective of this systematic review is to synthesise the best available evidence informing the effects of prosthetic socket design on outcomes of function, mobility, comfort and pain, energy expenditure, prosthetic and health related quality of life of adults with a trans-tibial amputation. Specifically, the review will consider studies evaluating the effects comparing the contemporary total surface bearing (TSB) socket design with the more traditional specific surface bearing (SSB) socket design.

\section{Background}


Mobility and function are often seen as primary indicators to successful rehabilitation for persons with a lower limb amputation ${ }^{1}$. Goal's of the medical rehabilitation team, including the prosthetist who is responsible for the design, manufacture and fitting of the limb prosthesis; are to provide care, education, equipment, resources and physical training to enable amputees the opportunity to reach a level of functional independence. The prosthesis, as an external attachment to the residuum (amputated stump), is a piece of equipment requiring custom manufacture to assist ambulatory mobility. Ambulatory mobility in the community setting can provide the amputee with a degree of regained independence ${ }^{2}$. Whether standing to prepare a meal, attending to self-care, returning to work or participating in high-level activities, mobility is a significant measure of ambulatory rehabilitation. Goals of rehabilitation and progressive community participation are established in collaboration with the amputee and the multi-disciplinary rehabilitation team, with prosthetic prescription largely determined by the prosthetist and the amputee.

The trans-tibial (or below-knee) prosthesis consists of a 'socket' and 'componentry'. The prosthetic socket is the surrounding encasement of the residuum, and the 'componentry' is the connecting hardware including foot/ankle module. Prescription indicators for componentry selection take into consideration patient ambulation potential or actual mobility, patient body weight, activities of daily living and greater activities of participation and recreation ${ }^{3}$. Typical prescription for the prosthetic socket is often more subjective and varies between countries, rehabilitation centres and individual clinicians. Clinical knowledge of the residuum with its physical tolerances, and the patients' ability to don and doff the prosthesis are factors considered during socket prescription. However lack of knowledge or inexperience with different socket techniques may be a barrier to introducing contemporary designs, as may cost of interface and equipment required for manufacture, time allowed for service provision or an amputee's accessibility to prosthetic service providers. The socket is a critical aspect of the prosthesis, specifically enabling weight bearing through the residuum. The socket design, providing fit and resultant function, is the key factor influencing prosthetic use by amputees, regardless of componentry used.

Objective outcome measures are increasingly used to justify clinical prescription through assessing mobility, function and quality of life of person's with an amputation using a prosthesis. ${ }^{4,5,6}$ Whilst there is a reasonable amount of literature evaluating effect of prosthetic componentry choice on functional outcomes for amputees ${ }^{7}$ there is a need for a review of the evidence relating to prosthetic socket design and the possible influence of socket design on health outcomes. This systematic review seeks to identify if there is a differing effect on health domains for people with amputation comparing two trans-tibial socket designs, the SSB with the TSB design, both of which are currently used throughout the world in prosthetic provision. 


\section{The SSB socket design}

Following World War 2 the need for prosthetic provision across Europe increased markedly and provided the stimulus for the design of the SSB trans-tibial socket, commonly known as patellar tendon bearing (PTB). Described in the 1950's by Radcliff $^{8}$, the PTB socket allowed persons with trans-tibial amputation to tolerate weight-bearing through the residuum. With pressure concentrated on the tolerant patellar tendon, the posterior musculature of the gastrocnemius/soleus and medioanteriorly on tibial flare; coupled with relief over bony prominences within the residuum $^{8}$, suspension of the prosthesis was achieved mostly via supracondylar cuff. The Patella Tendon Supracondular socket (PTS or PTB-SC), Patellar Tendon Kegel (PTK), Kondylen-Bein-Muenster (KBM) socket designs each follow a similar principle to the PTB with extra functional effect derived from extended proximal socket trimlines forming supracondylar self-suspension, increased medio-lateral stability of the knee joint, and improved cosmesis. ${ }^{9}$ This SSB socket design was an improvement on its predecessor the 'conventional' prosthesis consisting of proximal leather thigh corset, which allowed for minimal weight to be borne through the residuum, was heavy, cumbersome and restricted knee joint range of motion.

For the purposes of this review the SSB socket design will include descriptors PTB, PTS, PTB-SC, PTK or KBM socket designs. The SSB is usually manufactured with a foam interface liner fitting however may have a silicone or gel based liner within the rigid socket. The SSB is identified by the specific areas of pressure relayed to the residuum during casting, rectification and manufacture; and this design remains in popular use throughout the world today. Beneath the foam liner the amputee wears cotton or woollen sock/s directly onto the residuum, to assist with accommodating volume fluctuation and wicking away sweat to optimise socket fit and comfort. Advantages of the SSB include relative low cost to manufacture, ${ }^{10,11}$ readily available materials for use, and greater adjustability within socket foam interface to ensure fit for the changing residuum. ${ }^{9}$

\section{The TSB socket design}

In the early 1980's Ossur Kristinsson, a trans-tibial amputee and prosthetist, developed the Icelandic roll-on silicone socket (ICEROSS) liner, widely used in Iceland from 1986. ${ }^{12}$ Made from silicone and cylindrical in shape, the liner is inverted and rolls onto the residuum providing upward compression forces. ${ }^{12}$ The resultant intimate fit and the nature of silicone serves to provide a stabilising interface liner that offers skin and tissue protection from the prosthetic socket and external forces that are transferred upward through the residuum. ${ }^{13}$ The original ICEROSS liner incorporates an attachment in the external distal end, which a corrugated pin is secured to. The pin engages into a lock component fabricated into the distal end of the prosthetic socket, thus providing a secure connection between the silicone interface liner and the 
prosthetic socket, becoming the suspension mechanism. By the 1990's ICEROSS liners were introduced to the worldwide prosthetic community dissolving the need for supracondylar suspension for trans-tibial amputees. Advantages of using ICEROSS liners include a superior suspension system, stabilisation of soft tissue, minimal pistoning (stretching), helping to improve circulation and increase in comfort. ${ }^{13}$

To complement the ICEROSS system Ossur Kristinsson in 1993 described the TSB socket concept; where within the TSB socket weight was borne by the entire surface of the residuum and the socket design was used in collaboration with the silicone liner system. ${ }^{12}$ The TSB socket design was also described by Staats, advocating the hydrostatic principle achieved by suction suspension with or without silicone interface use. ${ }^{14}$ Essentially the TSB design relied on the hydrostatic principle of containing the entire mass of the residuum in an equal volume of socket with minimal movement/pistoning to keep the residuum in contact with the socket at all times during the gait cycle. ${ }^{12,14}$ The TSB design concept has spread to clinical practice and has been reported to have had varied results depending on residuum suitability, clinician knowledge, skill in fabrication, and suspension systems used in conjunction with the TSB design. ${ }^{10,13}$ Although at times described as a hydrostatic design, as the TSB socket commonly used in practice follows the hydrostatic principle of load transfer; in reality the socket uses a hybrid style of limited area loading combined with hydrostatic load transfer to cater for the changing mechanics of the human residuum throughout the gait cycle.

TSB socket design will be defined to include designs reported as TSB, hydrostatic, and limited area loading. All of these TSB socket descriptions utilise a silicon or gel interface liner achieving suspension through distal pin, lanyard or Velcro attachment; or a sealed system secured by expulsion valve, elevated vacuum, or membrane incorporated within the interface liner with or without the use of a knee sleeve. The TSB design can be differentiated from the SSB design as it lacks a foam interface liner, has no suspension via supracondylar means of cuff or straps, and has no specific increased pressure loading areas onto the residuum.

Adults may present as trans-tibial amputees for a variety of reasons. Vascular disease, trauma, cancer, infection and congenital reasons are the leading causes for amputation of the lower-limb ${ }^{15}$. Different countries and regions have varied numbers of amputees within each causal category. ${ }^{15}$ The trans-tibial prosthesis is the most commonly prescribed as the majority of lower-limb amputees have this level of amputation irrespective of aetiology. Although data is unavailable comparing prescription of TSB versus SSB transtibial prosthetic designs in Australia or elsewhere; this review aims to include as many studies comparing the two socket designs, regardless of reason for amputation, in order to represent the complement of amputees treated in most prosthetic clinics worldwide. 
This review seeks to retrieve studies using SSB and TSB socket designs where outcome measures were utilised, to quantify and indicate the effect on health domains for transtibial amputees. The use of domains relating to health can be somewhat complex depending on your area of interest and interpretation. For this review our terminology and understanding stems from World Health Organisation (WHO)'s International Classification of Disability and Health, known as ICF whereby health domains and health-related domains are areas of interest for measuring the effect of health both negative and positive on any set disease, diagnosis or disability group. The WHO domains relate to two categories 1) body functions and structures, and 2) activities and participation. ${ }^{16}$

The outcome measures in included studies are anticipated to be typical measures used in hospital, rehabilitation and community settings. Generic and amputee specific measures are expected, as these are reflective of measures used in every-day clinical practice. Essentially domains are 'what' we want to measure, as determined by patients and their medical teams as being relevant; and the outcome measures used relate to 'how' we are going to measure it.

In a preliminary search of PubMED, Scopus, and CINAHL one systematic review by Van der Linde et $\mathrm{al}^{7}$ was identified as having a reference to, though not a focus on, transtibial socket design. Studies retrieved for the review were published from $1996-$ 2001. Only one study in the review focused on the effect of socket design, and that study compared two suspension methods within the TSB socket design. No systematic reviews were identified comparing the effects of different trans-tibial socket designs.

Irrespective of the reasons for prescription choice, there is a need for a greater understanding of the effect using different socket designs in terms of patient rehabilitation, mobility, function and quality of life; so that the importance of socket design prescription is thoroughly considered during the prescription process. The aim of this systematic review of published and grey literature comparing SSB and TSB designs is to assist the prosthetic community with decisions and recommendations for prosthetic prescription through identification of evidence based indicators for socket design use, rather than relying on anecdotal, subjective preference or experimental reasons for prescription.

\section{Inclusion criteria}

\section{Types of participants}

This review will consider adult trans-tibial amputees 18 years or older, with acquired or congenital limb loss irrespective of the aetiology, gender or the presence of comorbidities using prostheses with either the contemporary total surface bearing (TSB) 
socket design or the traditional specific surface bearing (SSB) socket design. Each of the studies considered for inclusion will have documented study populations utilising both TSB and SSB socket designs within the study.

\section{Types of interventions}

The intervention of interest is the use of TSB socket. This is the 'hydro static' design or 'limited load bearing' often using silicon or gel liner interface, with suspension achieved via distal attachment of pin and lock or lanyard, expulsion valve and sleeve, vacuum assisted, integrated membrane seal or a combination of these. The comparative intervention will be the use of a SSB socket. This is often referred to as PTB, PTS, PTB-SC, PTK or KBM, typically utilising soft foam liner interface paired with supracondylar suspension.

\section{Types of outcomes}

This review will consider studies that include but will not be limited to the following outcomes:

- Function and Mobility measured for example using Amputee Activity Score (AAS) or Locomotor Capabilities Index (LCl-5) or Timed Walk Tests (TWTs) or Step Activity Monitor (SAM);

- Comfort and Pain measured for example using Socket Comfort Score (SCS) or Brief Pain Inventory (BPI);

- Quality of Life including: Prosthetic Related Quality of Life (PRQoL) measured for example using Prosthetic Evaluation Questionnaire (PEQ) and Health Related Quality of Life (HRQoL) measured for example using Trinity Amputation \& Prosthesis Experience Scales (TAPES) or Short Form 36 (SF-36);

- Energy Expenditure measured for example using Physiological Cost Index (PCI).

\section{Types of studies}

This review will consider both experimental and observational study designs including randomised controlled trials, non-randomised controlled trials, quasi-experimental, before and after studies, prospective and retrospective cohort studies, case control studies and analytical cross sectional studies for inclusion. It will also consider descriptive studies including case series and individual case reports for inclusion as long as participants have utilised both TSB and SSB designs within the study.

\section{Search strategy}

The search strategy aims to find both published and unpublished studies. A three-step search strategy will be utilised. An initial limited search of PubMED, Scopus and CINAHL will be undertaken followed by analysis of the text words contained in the title and abstract, and of the index terms used to describe each article. A second search 
using all identified keywords and index terms will then be undertaken across all included databases. Thirdly, the reference list of all identified reports and articles will be searched for additional studies.

Only studies published in English language will be considered for inclusion in this review. Studies published from inception of databases until present day (June 2012) are to be considered.

The databases to be searched include:

PubMED

CINAHL Plus

Scopus

Embase

PEDro

The search for unpublished studies will include:

Mednar,

Cochrane central register of controlled trials (CENTRAL),

Proquest Dissertations and Theses,

National Library of Australia's Trove service,

Electronic Thesis Online Service (EThOS),

Australian New Zealand Clinical Trial Registry (ANZCTR),

EU Clinical trials Registry (EU-CTR),

Clinical Trials.gov - USA trial registry,

The professional bodies of International Society of Prosthetics and Orthotics, the Australian Orthotic and Prosthetic Association (AOPA), and the American Academy of Orthotics and Prosthetics (AAOP) in partnership with oandp.com have resources such as websites, gazettes, conference and congress proceedings; and Conference Proceedings Citation Indexes (Web of Science) will also be explored for unpublished studies.

Initial keywords to be used will be:

'TSB', 'SSB', 'PTB', 'total surface bearing', 'specific surface bearing', 'patella tendon bearing', 'prosthetic', 'functional outcomes', 'socket design', 'amputee', 'trans-tibial' 'outcome measures', 'domains', 'lower-limb'.

\section{Assessment of methodological quality}


Papers selected for retrieval will be assessed by two independent reviewers for methodological validity prior to inclusion in the review using standardised critical appraisal instruments from the Joanna Briggs Institute Meta Analysis of Statistics Assessment and Review Instrument (JBI-MAStARI) (Appendix II). Any disagreements that arise between the reviewers will be resolved through discussion, or with a third reviewer.

\section{Data collection}

Data will be extracted from papers included in the review using the standardised data extraction tool from JBI-MAStARI (Appendix III). The data extracted will include details about the interventions, populations, study methods and outcomes of significance to the review question and specific objectives.

\section{Data synthesis}

Quantitative data will, where possible be pooled in statistical meta-analysis using JBIMAStARI. All results will be subject to double data entry. Effect sizes expressed as odds ratio (for categorical data) and weighted mean differences (for continuous data) and their $95 \%$ confidence intervals will be calculated for analysis. Heterogeneity will be assessed statistically using the standard Chi-square and also explored using subgroup analyses based on the different study designs included in the review. Where statistical pooling is not possible the findings will be presented in narrative form including tables and figures to aid in data presentation where appropriate.

\section{Conflicts of interest}

Nil.

\section{Acknowledgements}

This review forms part of the primary reviewer's submission for Masters of Clinical Sciences program, and as such a second reviewer is required only for assistance with critical appraisal. Edward Ko Ko Aung is acknowledged for his support to act as second reviewer for this systematic review.

\section{References}

[1] Sansam, K, Neumann, V, O'Connor, R, Bhakta, B. Predicting walking ability following lower limb amputation: a systematic review of the literature. J Rehabil Med.2009; 41: 593-603. 
[2] Klute, G K, Berge, J S, Orendurff, M S, Williams, R M, Czerniecki, J M. Prosthetic intervention effects on activity of lower-extremity amputees. Arch Phys Med Rehabil.2006; 87: 717-722.

[3] Gailey, R S, Roach, K E, Applegate, E B, Cho, B, Cunniffe, B, Licht, S, Maguire, M, Nash, M. The amputee mobility predictor: an instrument to assess determinants of the lower limb amputee's ability to ambulate. Arch of Phys Med Rehabil.2002; 83: 613627.

[4] Condie, E, Scott, H, Treweek, S. Lower limb prosthetic outcome measures: a review of the literature 1995 to 2005. Journal of Prosthetics and Orthotics.2006; 18(1S): 13-45.

[5] Penn-Barwell, J G. Outcomes in lower limb amputation following trauma: a systematic review and meta-analysis. Injury.2011; 42: 1474-1479.

[6] Samuelsson, K A M, Töytäri, O, Salminen, A-L, Brandt, Å. Effects of lower limb prosthesis on activity, participation, and quality of life: a systematic review. Prosthetics and Orthotics International.2012.

[7] van der Linde, H, Hofstad, C J, Geurts, A C H, Postema, K, Geertzen, J H B, van Limbeek, J. A systematic literature review of the effect of different prosthetic components on human functioning with a lower-limb prosthesis. Journal of Rehabilitation Research and Development.2004; 41(4): 555-570.

[8] Radcliffe, C W, Foort, J, University of California Berkeley, Biomechanics Laboratory. The patellar-tendon-bearing below-knee prosthesis. 1961.

[9] Fergason, J and Smith, D G. Socket considerations for the patient with a transtibial amputation. Clinical Orthopaedics and Related Research.1999; 361: 76-84.

[10] Coleman, K L, Boone, D A, Laing, L S, Mathews, D E, Smith, D G. Quantification of prosthetic outcomes: elastomeric gel liner with locking pin suspension versus polyethylene foam liner with neoprene sleeve suspension. Journal of Rehabilitation Research and Development.2004; 41(4): 591-602.

[11] Datta, D, Harris, I, Heller, B, Howitt, J, Martin, R. Gait, cost and time implications for changing from PTB to ICEX® sockets. Prosthetics and Orthotics International.2004; 28(2): 115-120.

[12] Kristinsson, O. The ICEROSS concept: a discussion of a philosophy. Prosthetics and Orthotics International.1993; 17: 49-55.

[13] Baars, E C T and Geertzen, J H B. Literature review of the possible advantages of silicon liner socket use in trans-tibial prostheses. Prosthetics and Orthotics International.2005; 291): 27-37. 
[14] Staats, T B and Lundt, J. The UCLA total surface bearing suction below-knee prosthesis. Clin Prosthet Orthot.1987; 11(3): 118-130.

[15] Esquenazi, A. Amputation rehabilitation and prosthetic restoration. From surgery to community reintegration. Disability \& Rehabilitation.2004; 26(14/15): 831-836.

[16] World Health Organisation. International classification of functioning disability and health. http://www.who.int/classifications/icf/en/.2012. 


\section{Appendix I: Joanna Briggs Institute critical appraisal instruments MAStARI Appraisal instrument}

\section{JBI Critical Appraisal Checklist for Randomised Control / Pseudo-randomised Trial}

Reviewer Date

Author Year Record Number

1. Was the assignment to treatment groups truly random?

2. Were participants blinded to treatment allocation?

3. Was allocation to treatment groups concealed from the allocator?

4. Were the outcomes of people who withdrew described and included in the analysis?

5. Were those assessing outcomes blind to the treatment allocation?

6. Were the control and treatment groups comparable at entry?

7. Were groups treated identically other than for the named interventions

8. Were outcomes measured in the same way for all groups?

9. Were outcomes measured in a reliable way?

10. Was appropriate statistical analysis used?

Overall appraisal: $\quad$ Include $\square \quad$ Exclude $\square \quad$ Seek further info. 


\section{JBI Critical Appraisal Checklist for Descriptive / Case Series}

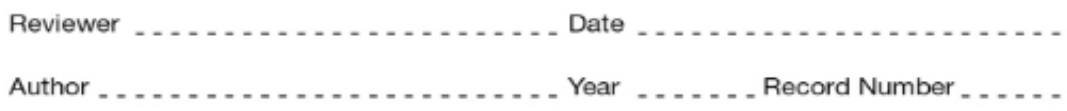

Author

Yes No Unclear Applicable

1. Was study based on a random or pseudorandom sample?

2. Were the criteria for inclusion in the sample clearly defined?

3. Were confounding factors identified and strategies to deal with them stated?

4. Were outcomes assessed using objective criteria?

5. If comparisons are being made, was there sufficient descriptions of the groups?

6. Was follow up carried out over a sufficient time period?

7. Were the outcomes of people who withdrew described and included in the analysis?

8. Were outcomes measured in a reliable way?

9. Was appropriate statistical analysis used?
Overall appraisal:
Include
Exclude
Seek further info

Comments (Including reason for exclusion 


\section{JBI Critical Appraisal Checklist for Comparable}

\section{Cohort/ Case Control}

Reviewer ................... Dat

Author _........... Year

Record Number

1. Is sample representative of patients in the population as a whole?

2. Are the patients at a similar point in the course of their condition/illness?

3. Has bias been minimised in relation to selection of cases and of controls?

4. Are confounding factors identified and strategies to deal with them stated?

5. Are outcomes assessed using objective criteria?

6. Was follow up carried out over a sufficient time period?

7. Were the outcomes of people who withdrew described and included in the analysis?

8. Were outcomes measured in a reliable way?

9. Was appropriate statistical analysis used? 
Appendix II: Joanna Briggs Institute data extraction instruments MAStARI data extraction instrument

\section{JBI Data Extraction Form for \\ Experimental / Observational Studies}

Reviewer
Author
Journal_
Study Method
RCT
Retrospective $\square$ Quasi-RCT $\square$ - $\square$ Lecord Number $\quad$ Observational $\square$

Participants

Setting

Population

Sample size

Group A Group B

Interventions

Intervention A

Intervention B

Authors Conclusions:

Reviewers Conclusions: 
Study results

Dichotomous data

\begin{tabular}{|l|l|l|}
\hline Outcome & $\begin{array}{c}\text { Intervention ( ) } \\
\text { number/total number }\end{array}$ & $\begin{array}{c}\text { Intervention ( ) } \\
\text { number/total number }\end{array}$ \\
\hline & & \\
\hline & & \\
\hline & & \\
\hline & & \\
\hline
\end{tabular}

Continuous data

\begin{tabular}{|l|l|l|}
\hline Outcome & $\begin{array}{c}\text { Intervention ( I J } \\
\text { number/ total number }\end{array}$ & $\begin{array}{c}\text { Intervention ( I J } \\
\text { number / total number }\end{array}$ \\
\hline & & \\
\hline & & \\
\hline & & \\
\hline & & \\
\hline & & \\
\hline
\end{tabular}




\section{Appendix II: Search Strategies}

\section{PubMED/ MEDLINE}

\begin{tabular}{|c|c|c|c|}
\hline Tibia & Amputation & Prosthesis & Type of socket \\
\hline $\begin{array}{l}\text { Transtibia* } \\
\text { OR } \\
\text { Tibia[mh] } \\
\text { OR } \\
\text { Tibia*[tw] } \\
\text { OR } \\
\text { Below knee*[tw] } \\
\text { OR } \\
\text { Lower extremit*[tw] } \\
\text { OR } \\
\text { Lower limb*[tw] }\end{array}$ & $\begin{array}{l}\text { Amputees[mh] } \\
\text { OR } \\
\text { ampute } e^{*}[\mathrm{tw}] \\
\text { OR } \\
\text { Amputation[mh:noexp] } \\
\text { OR } \\
\text { amputat*[tw] } \\
\text { OR } \\
\text { Reamputat*[tw] }\end{array}$ & $\begin{array}{l}\text { Prosthesis } \\
\text { design[mh:noexp] } \\
\text { OR } \\
\text { Prosthes*[tw] } \\
\text { OR } \\
\text { Prosthet*[tw] } \\
\text { OR } \\
\text { Artificial limb*[tw] } \\
\text { OR } \\
\text { Prostheses and } \\
\text { implants[mh:noexp] }\end{array}$ & $\begin{array}{l}\text { Surface bearing[tw] } \\
\text { OR } \\
\text { Hydrostatic[tw] } \\
\text { OR } \\
\text { Tendon bearing[tw] } \\
\text { OR } \\
\text { Ligament bearing[tw] } \\
\text { OR } \\
\text { Tsb[tw] } \\
\text { OR } \\
\text { Ptb[tw] } \\
\text { OR } \\
\text { ssb[tw] }\end{array}$ \\
\hline
\end{tabular}

Logic Grid for PubMED MEDLINE search:

\section{Search date: $9^{\text {th }}$ September 2012}

\#1 Transtibia*[tw] OR Tibia[mh] OR Tibia*[tw] OR Below knee*[tw] OR Lower extremit*[tw] OR Lower limb*[tw]

$=131933$ studies identified

\#2 Amputees[mh] OR amputee*[tw] OR Amputation[mh:noexp] OR amputat*[tw] OR Reamputat*[tw]

$=37153$ studies identified

\#3 Prosthesis design[mh:noexp] OR Prosthes*[tw] OR Prothet*[tw] OR Artificial limb*[tw] OR Prostheses and implants[mh:noexp]

$=210389$ studies identified 
\#4 Surface bearing[tw] OR Hydrostatic[tw] OR Tendon bearing[tw] OR Ligament bearing[tw] OR Tsb[tw] OR Ptb[tw] OR ssb[tw]

$=16189$ studies identified

\#1 AND \#2 AND \#3 AND \#4 when combined

$=75$ total studies identified 


\section{Embase}

\begin{tabular}{|l|l|l|}
\hline Trans-Tibial & Prosthesis & Type of socket \\
\hline 'below knee amputation':de,ab,ti & 'leg prosthesis':de,ab,ti & hydrostatic*aab,ti \\
OR & OR & OR \\
((amputation/exp & 'artificial leg':ab,ti & 'patella tendon bearing':ab,ti \\
OR & OR & OR \\
Amputation*:ab,ti) & prosthes*:ab,ti & 'total surface bearing':ab,ti \\
AND & OR & OR \\
('below knee':ab,ti & prosthet*:ab,ti & Patella*:ab,ti \\
OR & & OR \\
Leg:ab,ti & & Ptb:ab,ti \\
OR & & OR \\
Tibia*:ab,ti & & Tsb:ab,ti \\
OR & & \\
'lower extremity':ab,ti)) & & \\
\hline
\end{tabular}

Logic Grid for Embase search:

\section{Search date: $15^{\text {th }}$ September 2012}

\#1 'below knee amputation':de,ab,ti OR ((amputation/exp OR Amputation*:ab,ti) AND ('below knee':ab,ti OR Leg:ab,ti OR Tibia*:ab,ti OR 'lower extremity':ab,ti))

$=11259$ studies identified

\#2 'leg prosthesis':de,ab,ti OR 'artificial leg':ab,ti OR prosthes*:ab,ti OR prosthet*:ab,ti

$=101110$ studies identified

\#3 hydrostatic*:ab,ti OR 'patella tendon bearing':ab,ti OR 'total surface bearing':ab,ti OR

Patella*:ab,ti OR Ptb:ab,ti OR Tsb:ab,ti

$=28205$ studies identified

\#1 AND \#2 AND \#3 when combined

$=122$ total studies identified. 
CINAHL Plus

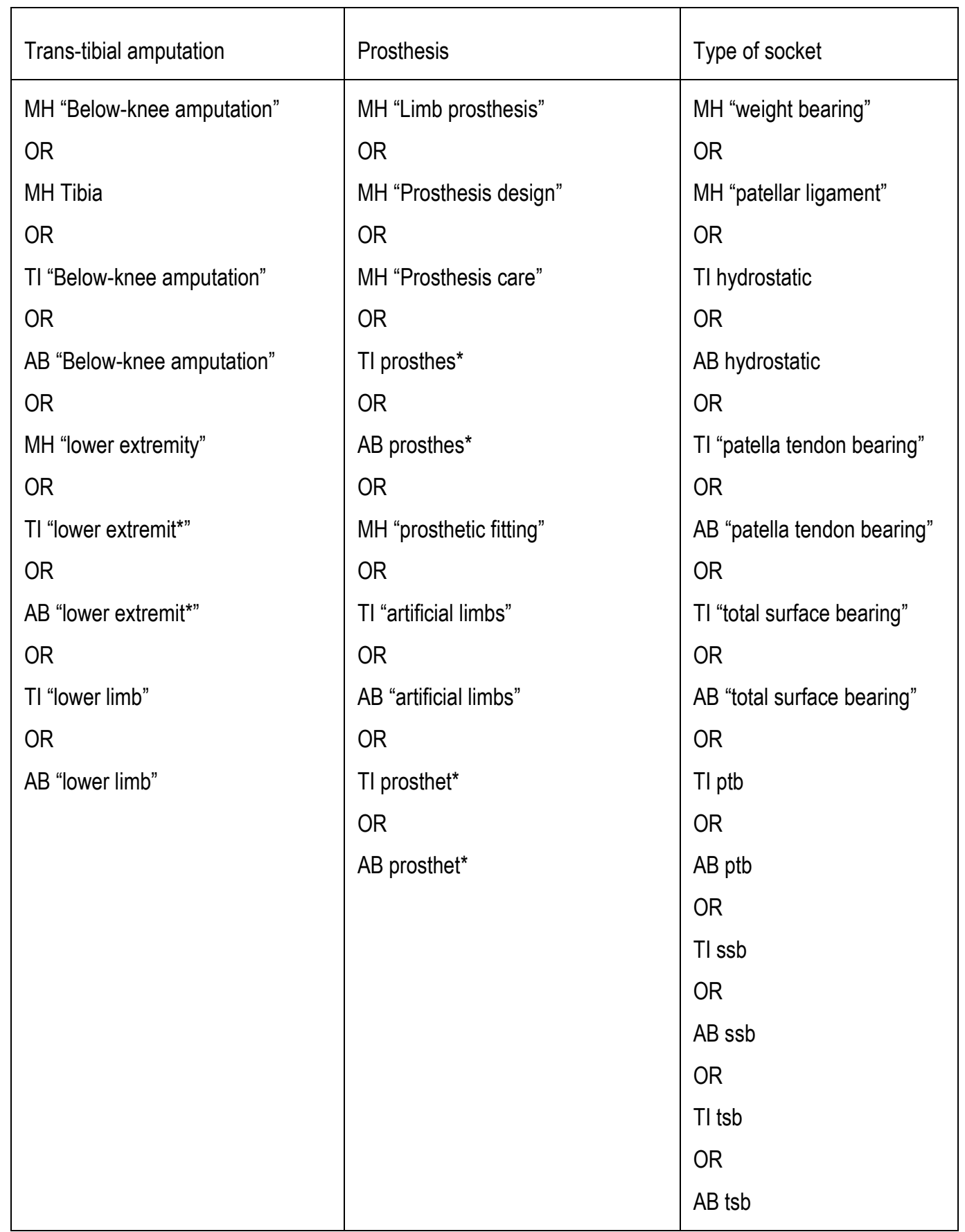

\section{Logic Grid for CINAHL Plus search:}

\section{Search date: $15^{\text {th }}$ September 2012}

\#1 MH "Below-knee amputation" OR MH Tibia OR TI "Below-knee amputation" OR AB "Below-knee amputation" OR MH "lower extremity" OR TI "lower extremit" OR AB "lower extremit"” OR TI "lower limb" OR AB "lower limb" 
$=12232$ studies identified

\#2 MH "Limb prosthesis" OR MH "Prosthesis design" OR MH "Prosthesis care" OR TI prosthes* OR AB prosthes* OR MH "prosthetic fitting" OR TI "artificial limbs" OR AB "artificial limbs" OR

TI prosthet* OR AB prosthet*

$=7161$ studies identified

\#3 MH "weight bearing" OR MH "patellar ligament" OR TI hydrostatic OR AB hydrostatic OR TI "patella tendon bearing" OR AB "patella tendon bearing" OR TI "total surface bearing" OR AB "total surface bearing" OR TI ptb OR AB ptb OR TI ssb OR AB ssb OR TI tsb OR AB tsb

$=3148$ studies identified

\#1 AND \#2 AND \#3 when combined

$=36$ total studies identified. 


\section{Scopus}

\begin{tabular}{|c|c|c|c|}
\hline Tibia & Amputation & Prosthesis & Type of socket \\
\hline Transtibia* & Amputees & Prosthesis design & Surface bearing \\
\hline OR & OR & OR & OR \\
\hline Tibia* & amputee* & Prosthes* & Hydrostatic \\
\hline OR & OR & OR & OR \\
\hline Below knee* & Amputation & Prosthet ${ }^{*}$ & Tendon bearing \\
\hline OR & OR & OR & OR \\
\hline Lower extremit* & amputat* & Artificial limb* & Ligament bearing \\
\hline OR & OR & & OR \\
\hline \multirow[t]{5}{*}{ Lower limb* } & Reamputat ${ }^{*}$ & & Tsb \\
\hline & & & OR \\
\hline & & & Ptb \\
\hline & & & OR \\
\hline & & & $s s b$ \\
\hline
\end{tabular}

Logic Grid for Scopus search:

\section{Search date: 9th September 2012}

\#1 Transtibia* OR Tibia* OR Below knee* OR Lower extremit* OR Lower limb*

$=7960$ studies identified

\#2 Amputees OR amputee* OR Amputation OR amputat ${ }^{\star}$ OR Reamputat ${ }^{\star}$

$=50415$ studies identified

\#3 Prosthesis design OR Prosthes* OR Prothet* OR Artificial limb*

$=13377$ studies identified

\#4 Surface bearing OR Hydrostati] OR Tendon bearing OR Ligament bearing OR Tsb OR Ptb OR ssb

$=41896$ studies identified

\#1 AND \#2 AND \#3 AND \#4 when combined

$=19$ total studies identified. 


\section{Proquest Dissertations and Theses}

\begin{tabular}{|c|c|c|c|}
\hline Tibia & Amputation & Prosthesis & Type of socket \\
\hline Transtibia* & Amputees[mh] & Prosthesis design[mh:noexp] & Surface bearing[tw] \\
\hline OR & OR & OR & OR \\
\hline Tibia[mh] & amputee*[tw] & Prosthes* ${ }^{*}[\mathrm{tw}]$ & Hydrostatic[tw] \\
\hline OR & OR & OR & OR \\
\hline Tibia*[tw] & Amputation[mh:noexp] & Prosthet ${ }^{\star}[\mathrm{tw}]$ & Tendon bearing[tw] \\
\hline OR & OR & OR & OR \\
\hline Below knee $[$ tw $]$ & amputat* ${ }^{*}$ tw] & Artificial limb*[tw] & Ligament bearing[tw] \\
\hline OR & OR & OR & OR \\
\hline Lower & Reamputat $^{*}[\mathrm{tw}]$ & Prostheses & $\mathrm{Tsb}[\mathrm{tw}]$ \\
\hline 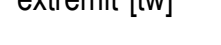 & & & OR \\
\hline $\mathrm{OR}$ & & & Ptb[tw] \\
\hline Lower limb*[tw] & & & OR \\
\hline & & & $\mathrm{ssb}[\mathrm{tw}]$ \\
\hline
\end{tabular}

Logic Grid for Proquest Dissertations and Theses search:

\section{Search date: 16 ${ }^{\text {th }}$ September 2012}

\#1 Transtibia*[tw] OR Tibia[mh] OR Tibia*[tw] OR Below knee*[tw] OR Lower extremit*[tw] OR Lower limb*[tw]

$=115$ studies identified

\#2 Amputees[mh] OR amputee*[tw] OR Amputation[mh:noexp] OR amputat*[tw] OR Reamputat* $[\mathrm{tw}]$

$=1057$ studies identified

\#3 Prosthesis design[mh:noexp] OR Prosthes*[tw] OR Prothet ${ }^{*}[\mathrm{tw}]$ OR Artificial limb*[tw] OR Prostheses and implants[mh:noexp]

$=1$ study identified

\#4 Surface bearing[tw] OR Hydrostatic[tw] OR Tendon bearing[tw] OR Ligament bearing[tw] OR Tsb[tw] OR Ptb[tw] OR ssb[tw] 
$=86060$ studies identified

\#1 AND \#2 AND \#4 when combined

$=52$ total studies identified .

PEDro

Search date: $16^{\text {th }}$ September 2012

Amput $^{*}=60$ studies identified

\section{Mednar}

Search date: $15^{\text {th }}$ September 2012

\#1 ('trans-tibial' OR 'below-knee') AND (prosthe* OR 'patella tendon bearing' OR 'total surface bearing') $=383$ studies identified

Cochrane central register of controlled trials (CENTRAL)

Search date: $16^{\text {th }}$ September 2012

\#1 (trans-tibial OR transtibial OR 'below knee' OR below-knee) AND ('socket design' OR 'total surface bearing' OR 'patella tendon bearing' OR ptb OR tsb) $=66$ studies identified

Electronic Thesis Online Service (EThOS),

Search date: 16th September 2012

0 studies identified.

Australian New Zealand Clinical Trial Registry (ANZCTR),

Search date: 16th September 2012

Amput $^{*}=60$ studies identified 
0 studies identified.

\section{EU Clinical trials Registry (EU-CTR),}

Search date: $16^{\text {th }}$ September 2012

0 studies identified.

\section{National Library of Australia's Trove service,}

Search date: $16^{\text {th }}$ September 2012

0 studies identified.

Clinical Trials.gov - USA trial registry

Search date: $16^{\text {th }}$ September 2012

'transtibial'= 27 studies identified

The websites, gazettes, conference and congress proceedings of the professional bodies of International Society of Prosthetics and Orthotics (ISPO), the Australian Orthotic and Prosthetic Association Inc. (AOPA), and the American Academy of Orthotists and Prosthetists (AAOP), and Conference Proceedings Citation Indexes (Web of Science) and Science direct were explored for unpublished studies.

Initial keywords were:

'TSB', 'SSB', 'PTB', 'total surface bearing', 'specific surface bearing', 'patella tendon bearing', 'prosthetic', 'functional outcomes', 'socket design', 'amputee', 'trans-tibial' 'outcome measures', 'domains', 'lower-limb'.

American Academy of Orthotists and Prosthetists: Journal of Prosthetics and Orthotics JPO online

http://www.oandp.org/jpo/

Search date: $16^{\text {th }}$ September 2012 
$=2$ studies identified.

Conference Proceedings Citation Indexes (Web of Science)

Search date: $16^{\text {th }}$ September 2012

=21 proceedings identified

Science direct

http://www.sciencedirect.com/science/jrnlallbooks/sub/medicinedentistry/a

Search date: $16^{\text {th }}$ September 2012

$=1$ study identified 
Appendix III: Joanna Briggs Institute Critical Appraisal Instruments

MAStARI Critical appraisal instrument

JBI Critical Appraisal Checklist for Randomised

Control / Pseudo-randomised Trial

Reviewer

Date

Author

Year

Record Number

1. Was the assignment to treatment groups truly random?

2. Were participants blinded to treatment allocation?

3. Was allocation to treatment groups concealed from the allocator?

4. Were the outcomes of people who withdrew described and included in the analysis?

5. Were those assessing outcomes blind to the treatment allocation?

6. Were the control and treatment groups comparable at entry?

7. Were groups treated identically other than for the named interventions

8. Were outcomes measured in the same way for all groups?

9. Were outcomes measured in a reliable way?

10. Was appropriate statistical analysis used?

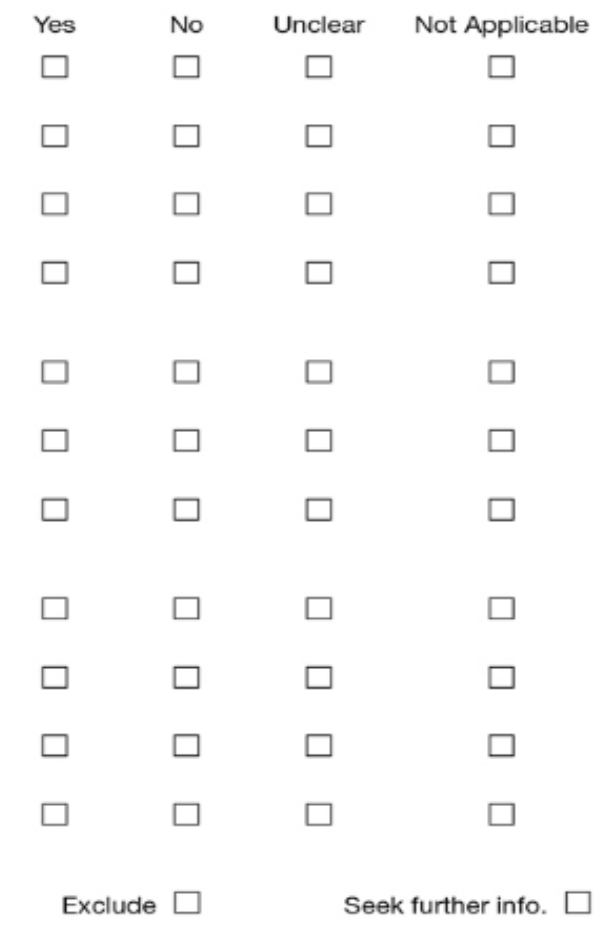


JBI Critical Appraisal Checklist for Descriptive / Case Series

Reviewer .............. Date

Author . . . . Year

Record Number . .......

Yes No Unclear Applicable

1. Was study based on a random or pseudorandom sample?

2. Were the criteria for inclusion in the sample clearly defined?

3. Were confounding factors identified and strategies to deal with them stated?

4. Were outcomes assessed using objective criteria?

5. If comparisons are being made, was there sufficient descriptions of the groups?

6. Was follow up carried out over a sufficient time period?

7. Were the outcomes of people who withdrew described and included in the analysis?

8. Were outcomes measured in a reliable way?

9. Was appropriate statistical analysis used?
Overall appraisal:
Include
Exclude
Seek further info

Comments (Including reason for exclusion) 
Appendix IV: Joanna Briggs Institute MAStARI data extraction instruments

\section{JBI Data Extraction Form for Experimental / Observational Studies}

Reviewer
Author _.
Journal_
Study Method
RCT $\quad$ Quasi-RCT $\quad \square$
Retrospective $\square$ Observational $\square$
Participants
Setting

Population

Sample size

Group A Group B

Interventions

Intervention A

Intervention B

Authors Conclusions:

Reviewers Conclusions: 
Study results

Dichotomous data

\begin{tabular}{|l|l|l|}
\hline Outcome & $\begin{array}{c}\text { Intervention ( } \\
\text { number/total number }\end{array}$ & $\begin{array}{c}\text { Intervention ( } \\
\text { number/total number }\end{array}$ \\
\hline & & \\
\hline & & \\
\hline & & \\
\hline & & \\
\hline
\end{tabular}

Continuous data

\begin{tabular}{|l|l|l|}
\hline Outcome & $\begin{array}{c}\text { Intervention ( ) } \\
\text { number/total number }\end{array}$ & $\begin{array}{c}\text { Intervention ( ) } \\
\text { number/total number }\end{array}$ \\
\hline & & \\
\hline & & \\
\hline & & \\
\hline & & \\
\hline
\end{tabular}




\section{Appendix V: Studies selected for retrieval $(n=26)$}

Ali S, Abu Osman NA, Naqshbandi MM, Eshraghi A, Kamyab M, Gholizadeh H. Qualitative study of prosthetic suspension systems on trans-tibial amputees' satisfaction and perceived problems with their prosthetic devices. Archives of physical medicine and rehabilitation. 2012;93(11):1919-1923.

Ali S, Osman NA, Mortaza N, Eshraghi A, Gholizadeh H, Wan Abas WA. Clinical investigation of the interface pressure in the trans-tibial socket with Dermo and Seal-In X5 liner during walking and their effect on patient satisfaction. Clinical biomechanics. 2012.

Astrom I, Stenstrom A. Effect on gait and socket comfort in unilateral trans-tibial amputees after exchange to a polyurethane concept. Prosthetics and orthotics international. 2004;28(1):28-36.

Baars EC, Geertzen JH. Literature review of the possible advantages of silicone liner socket use in trans - tibial prostheses. Prosthetics and orthotics international. 2005; (1):2737. Available from: http://onlinelibrary.wiley.com/o/cochrane/cldare/articles/DARE12005000697/frame.html.

Boonstra AM, van Duin W, Eisma W. International forum. Siliconee suction socket (3S) versus supracondylar PTB prosthesis with pelite liner: trans-tibial amputees' preferences. Journal of prosthetics \& orthotics. 1996;8(3):96-9.

Buis AWP, Blair A, Convery P, Sockalingam S, McHugh B. Pilot study: Data-capturing consistency of two trans-tibial casting concepts, using a manikin stump model: a comparison between the hands-on PTB and hands-off ICECAST Compact concepts. Prosthetics and orthotics international. 2003;27(2):100-6.

Coleman KL, Boone DA, Laing LS, Mathews DE, Smith DG. Quantification of prosthetic outcomes: elastomeric gel liner with locking pin suspension versus polyethylene foam liner with neoprene sleeve suspension. Journal of rehabilitation research and development. 2004;41(4):591-602. 
Convery P, Buis AWP. Socket/stump interface dynamic pressure distributions recorded during the prosthetic stance phase of gait of a trans-tibial amputee wearing a hydrocast socket. Prosthetics and orthotics international. 1999;23(2):107-12.

Datta D, Harris I, Heller B, Howitt J, Martin R. Gait, cost and time implications for changing from PTB to ICEX® sockets. Prosthetics and orthotics international. 2004;28(2):115-20.

Datta D, Vaidya SK, Howitt J, Gopalan L. Outcome of fitting an ICEROSS prosthesis: views of trans-tibial amputees. Prosthetics and orthotics international. 1996;20(2):111-5.

Dumbleton T, Buis AW, McFadyen A, McHugh BF, McKay G, Murray KD. Dynamic interface pressure distributions of two trans-tibial prosthetic socket concepts. Journal of rehabilitation research and development. 2009;46(3):405-15.

Engsberg JR, Sprouse SW, Uhrich ML, Ziegler BR, Luitjohan FD. Comparison of rectified and unrectified sockets for trans-tibial amputees. Journal of prosthetics and orthotics. 2006;18( 1):1-7.

Fergason J, Smith DG. Socket considerations for the patient with a trans-tibial amputation. Clinical orthopaedics and related research. 1999(361):76-84.

Goh JCH, Lee PVS, Chong SY. Comparative study between patellar-tendon-bearing and pressure cast prosthetic sockets. Journal of rehabilitation research and development. 2004;41(3B):491-502.

Hatfield AG, Morrison JD. Polyurethane gel liner usage in the Oxford Prosthetic Service. Prosthetics and orthotics international. 2001;25(1):41-6.

Hachisuka K, Dozono K, Ogata H, Ohmine S, Shitama H, Shinkoda K. Total surface bearing below-knee prosthesis: advantages, disadvantages, and clinical implications. Archives of physical medicine and rehabilitation. 1998;79( 7):783-9.

Kahle JT. Conventional and hydrostatic trans-tibial interface comparison. Journal of prosthetics \& orthotics. 1999;11(4):85-91. 
Klute GK, Berge JS, Biggs W, Pongnumkul S, Popovic Z, Curless B. Vacuum-assisted socket suspension compared with pin suspension for lower extremity amputees: effect on fit, activity, and limb volume. Archives of physical medicine and rehabilitation. 2011;92(10):1570-5.

Klute GK, Glaister BC, Berge JS. Prosthetic liners for lower limb amputees: a review of the literature. Prosthetics and orthotics international. 2010;34(2):146-53.

Laing S, Lee PV, Goh JC. Engineering a trans-tibial prosthetic socket for the lower limb amputee. Annals of the Academy of Medicine Singapore. 2011;40(5):252-9.

Manucharian SR. An investigation of comfort level trend differences between the hands-on patellar tendon bearing and hands-off hydrocast trans-tibial prosthetic sockets. Journal of prosthetics and orthotics. 2011;23(3):124.

Narita H, Yokogushi K, Shii S, Kakizawa M, Nosaka T. Suspension effect and dynamic evaluation of the total surface bearing (TSB) trans-tibial prosthesis: a comparison with the patellar tendon bearing (PTB) trans-tibial prosthesis. Prosthetics and orthotics international. 1997;21(3):175-8.

Normann E, Olsson A, Brodtkorb TH. Modular socket system versus traditionally laminated socket: a cost analysis. Prosthetics and orthotics international. 2011;35(1):76-80.

Sanders JE, Fatone S. Residual limb volume change: systematic review of measurement and management. Journal of rehabilitation research and development. 2011:48(8):949-86.

Selles RW, Janssens PJ, Jongenengel CD, Bussmann JB. A randomised controlled trial comparing functional outcome and cost efficiency of a total surface-bearing socket versus a conventional patellar tendon-bearing socket in trans-tibial amputees. Archives of physical medicine and rehabilitation. 2005;86(1):154-61.

Yiğiter K, Şener G, Bayar K. Comparison of the effects of patellar tendon bearing and total surface bearing sockets on prosthetic fitting and rehabilitation. Prosthetics and orthotics international. 2002;26(3):206-12. 


\section{Appendix VI: Studies excluded after review of full-text}

\section{Excluded studies $n=14$}

Ali S, Osman NA, Mortaza N, Eshraghi A, Gholizadeh H, Wan Abas WA. Clinical investigation of the interface pressure in the trans-tibial socket with Dermo and Seal-In X5 liner during walking and their effect on patient satisfaction. Clinical biomechanics. 2012.

Reason for exclusion: This paper did not address outcome measures relating to review.

Ali S, Abu Osman NA, Naqshbandi MM, Eshraghi A, Kamyab M, Gholizadeh H. Qualitative study of prosthetic suspension systems on trans-tibial amputees' satisfaction and perceived problems with their prosthetic devices. Archives of physical medicine and rehabilitation. 2012;93(11):1919-1923.

Reason for exclusion: This paper did not address outcome measures relating to review.

Baars EC, Geertzen JH. Literature review of the possible advantages of silicone liner socket use in trans - tibial prostheses. Prosthetics and orthotics international. 2005; (1):27-

37. Available from: http://onlinelibrary.wiley.com/o/cochrane/cldare/articles/DARE12005000697/frame.html.

Reason for exclusion: This paper was not a primary research study and did not meet the inclusion criteria.

Boonstra AM, van Duin W, Eisma W. International forum. Siliconee suction socket (3S) versus supracondylar PTB prosthesis with pelite liner: trans-tibial amputees' preferences. Journal of prosthetics \& orthotics. 1996;8(3):96-9.

Reason for exclusion: This paper did not address outcome measures relating to review.

Buis AWP, Blair A, Convery P, Sockalingam S, McHugh B. Pilot study: Data-capturing consistency of two trans-tibial casting concepts, using a manikin stump model: a 
comparison between the hands-on PTB and hands-off ICECAST Compact concepts. Prosthetics and orthotics international. 2003;27(2):100-6.

Reason for exclusion: This paper did not address outcome measures relating to review.

Convery P, Buis AWP. Socket/stump interface dynamic pressure distributions recorded during the prosthetic stance phase of gait of a trans-tibial amputee wearing a hydrocast socket. Prosthetics and orthotics international. 1999;23(2):107-12.

Reason for exclusion: This paper did not address outcome measures relating to review.

Dumbleton T, Buis AW, McFadyen A, McHugh BF, McKay G, Murray KD. Dynamic interface pressure distributions of two trans-tibial prosthetic socket concepts. Journal of rehabilitation research and development. 2009;46(3):405-15.

Reason for exclusion: This paper did not address outcome measures relating to review.

Fergason J, Smith DG. Socket considerations for the patient with a trans-tibial amputation. Clinical orthopaedics and related research. 1999(361):76-84.

Reason for exclusion: This paper did not address outcome measures relating to review.

Hatfield AG, Morrison JD. Polyurethane gel liner usage in the Oxford Prosthetic Service. Prosthetics and orthotics international. 2001;25(1):41-6.

Reason for exclusion: This paper did not address outcome measures relating to review.

Klute GK, Glaister BC, Berge JS. Prosthetic liners for lower limb amputees: a review of the literature. Prosthetics and orthotics international. 2010;34(2):146-53.

Reason for exclusion: This paper did not address outcome measures relating to review and was not a primary research study. 
Laing S, Lee PV, Goh JC. Engineering a trans-tibial prosthetic socket for the lower limb amputee. Annals of the Academy of Medicine Singapore. 2011;40(5):252-9.

Reason for exclusion: This paper did not address outcome measures relating to review.

Narita H, Yokogushi K, Shii S, Kakizawa M, Nosaka T. Suspension effect and dynamic evaluation of the total surface bearing (TSB) trans-tibial prosthesis: a comparison with the patellar tendon bearing (PTB) trans-tibial prosthesis. Prosthetics and orthotics international. 1997;21(3):175-8.

Reason for exclusion: This paper did not address outcome measures relating to review.

Normann E, Olsson A, Brodtkorb TH. Modular socket system versus traditionally laminated socket: a cost analysis. Prosthetics and orthotics international. 2011;35(1):76-80.

Reason for exclusion: This paper did not address outcome measures relating to review.

Sanders JE, Fatone S. Residual limb volume change: systematic review of measurement and management. Journal of rehabilitation research and development. 2011;48(8):949-86.

Reason for exclusion: This paper did not address outcome measures relating to review. Not a primary study.

\section{Studies excluded after critical appraisal:}

$n=2$

Goh JCH, Lee PVS, Chong SY. Comparative study between patellar-tendon-bearing and pressure cast prosthetic sockets. Journal of rehabilitation research and development. 2004;41(3B):491-502.

Reason for exclusion: No acclimatisation period for new sockets, specifically 15 
minutes allowed for acclimatisation, with outcome measures tested on same day for both sockets. Absence of interface liners on both sockets not reflective of typical clinical prescription.

Kahle JT. Conventional and hydrostatic trans-tibial interface comparison. Journal of prosthetics \& orthotics. 1999;11(4):85-91.

Reason for exclusion: Minimal acclimatisation period for socket designs, 1 week, and not consistent across all subjects. Socket preference was the only outcome of interest, which was compromised by incomplete data and minimal acclimatisation. 


\section{Appendix VII: Characteristics of included studies}

\begin{tabular}{|c|c|c|c|c|c|c|}
\hline Citation & Setting & Participants & Methods & $\begin{array}{l}\text { Intervention } \\
\text { I } \\
\text { Comparator }\end{array}$ & $\begin{array}{l}\text { Outcome } \\
\text { Measures }\end{array}$ & Main Findings \\
\hline $\begin{array}{l}\text { Selles et al } \\
2005^{52} \\
\text { Experimental }\end{array}$ & $\begin{array}{l}\text { Outpatient } \\
\text { hospital } \\
\text { setting and } \\
\text { prosthetic } \\
\text { facility in The } \\
\text { Netherlands }\end{array}$ & $\begin{array}{l}\mathrm{n}=26 \\
\text { Aetiology: } \\
\text { V/T/O: } \\
\text { 14/11/1 } \\
\text { Mean age: } \\
63 \text { years } \\
\text { Walking > } 1 \\
\text { year. }\end{array}$ & $\begin{array}{l}\text { Prospective } \\
\text { RCT 2-arm } \\
\text { design. } \\
\text { Assessed } \\
\text { baseline \& } 3 \\
\text { months } \\
\text { acclimatisation. }\end{array}$ & $\begin{array}{l}\text { TSB: GI: PS } \\
\text { (ICEX) } \\
n=12 \\
\text { SSB: GI: PS } \\
n=14\end{array}$ & $\begin{array}{l}\text { Function: } \\
\text { Walk speed } \\
\text { Stride length } \\
\text { Gait-asymmetries } \\
\text { ADL analysis } \\
\text { QoL: } \\
\text { Satisfaction } \\
\text { Preference } \\
\text { Yes/No } \\
\text { Comfort \& Pain } \\
\text { Phantom pain \& } \\
\text { pain (PEQ) } \\
\text { Costs: } \\
\text { Time } \\
\text { Material } \\
\text { Other: } \\
\text { Prosthetic Mass }\end{array}$ & $\begin{array}{l}\text { No difference in gait } \\
\text { parameters } \\
p=0.502 \\
p=0.704 \\
\text { Temporal } p=0.436 \\
\text { Spatial } p=0.889 \\
\text { No difference } \\
\text { No difference in } \\
\text { QoL domain } \\
p=0.524 \\
\text { TSB: } 11 / 12, \\
S S B: 13 / 14 \\
\text { No difference in } \\
\text { comfort domain } \\
p=0.774 \\
p=0.475 \\
\text { Economic variables } \\
\text { significant } \\
\text { SSB longer delivery } \\
\text { ( } p=0.001 \text { ), } \\
\text { TSB higher } \\
\text { material } \$ \text { ( } p=0.001 \text { ) } \\
\text { No significant } \\
\text { difference ( } p=0.066 \text { ) }\end{array}$ \\
\hline $\begin{array}{l}\text { Coleman et al } \\
2004^{23} \\
\text { Experimental }\end{array}$ & $\begin{array}{l}\text { USA } \\
\text { outpatient } \\
\text { setting }\end{array}$ & $\begin{array}{l}\mathrm{n}=13 \\
\text { Aetiology: } \\
\text { V/T/O: } \\
0 / 13 / 0 \\
\text { Mean age: } \\
49 \text { years } \\
>1 \text { year } \\
\text { post- } \\
\text { amputation. } \\
\text { Activity K2+ }\end{array}$ & $\begin{array}{l}\text { Prospective } \\
\text { RCT cross- } \\
\text { over design. } \\
\text { Assessed after } \\
2.5 \text { months } \\
\text { acclimatisation. } \\
\text { Limitation: } \\
\text { Subjects did } \\
\text { not use new } \\
\text { socket design } \\
\text { exclusively. }\end{array}$ & $\begin{array}{l}\text { TSB: GI: PS } \\
\text { SSB: FI: SC- } \\
\text { S }\end{array}$ & $\begin{array}{l}\text { Function: } \\
\text { Stride length } \\
\text { Step-count } \\
\text { Wear-time } \\
\text { Ambulation (PEQ) } \\
\text { QoL: } \\
\text { PEQ 7 subscales } \\
\text { Preference } \\
\text { Comfort \& pain: }\end{array}$ & $\begin{array}{l}\begin{array}{l}\text { Favoured SSB in } \\
\text { community }\end{array} \\
\text { No difference, } \\
(p=0.9048) \\
\text { SSB } 83 \% \uparrow \\
(p=0.002) \\
\text { SSB } 82 \% \\
\uparrow(p=0.018) \\
\text { No difference, } \\
(p=0.4143) \\
\text { Selection favoured } \\
\text { SSB design } \\
\text { No difference } \\
\text { TSB: } 3 / 13 \text {, SSB: } \\
\text { 10/13 } \\
\text { No difference in } \\
\text { comfort domain }\end{array}$ \\
\hline
\end{tabular}




\begin{tabular}{|c|c|c|c|c|c|c|}
\hline & & & & & $\begin{array}{l}\text { SCS } \\
\text { BPI } 13 \text { questions } \\
\text { RLH (PEQ) }\end{array}$ & $\begin{array}{l}\text { No difference, } \\
(p=0.7344) \\
\text { No difference } \\
\text { No difference, } \\
(p=0.8926)\end{array}$ \\
\hline $\begin{array}{l}\text { Datta et al } \\
2004^{24} \\
\text { Experimental }\end{array}$ & $\begin{array}{l}\text { Outpatient } \\
\text { hospital } \\
\text { setting and } \\
\text { prosthetic } \\
\text { facility in } \\
\text { England. }\end{array}$ & $\begin{array}{l}\mathrm{n}=21 \\
\text { Aetiology: } \\
\text { V/T/O: } \\
7 / 10 / 4 \\
\text { Mean age: } \\
51 \text { yrs }\end{array}$ & $\begin{array}{l}\text { Prospective } \\
\text { RCT 2-arm } \\
\text { design. } \\
\text { Assessed at } \\
\text { baseline \& } 6 \\
\text { weeks } \\
\text { acclimatisation. }\end{array}$ & $\begin{array}{l}\text { TSB: GI: PS } \\
\text { (ICEX) n=11 } \\
\text { SSB: FI: SC- } \\
\text { S } \\
n=10\end{array}$ & $\begin{array}{l}\text { Function: } \\
\text { Walk speed } \\
\text { Stride length } \\
\text { Cadence } \\
\text { Gait-asymmetries } \\
\text { Costs: } \\
\text { Time } \\
\text { Material }\end{array}$ & $\begin{array}{l}\text { No difference in gait } \\
\text { parameters } \\
\text { No difference, } \\
(p=0.369) \\
\text { No difference, } \\
\text { ( } p=0.314) \\
\text { No difference, } \\
\text { ( } p=0.605) \\
\text { Temporal } p=0.282 \\
\text { Spatial } p=0.085 \\
\text { Economic variables } \\
\text { significant } \\
\text { SSB } 2.5 \text { times } \\
\text { longer delivery } \\
\text { TSB } 2.5 \text { times more } \\
\text { expensive }\end{array}$ \\
\hline $\begin{array}{l}\text { Klute et al } \\
2011^{51} \\
\text { Experimental }\end{array}$ & USA & $\begin{array}{l}n=5 \\
\text { Aetiology: } \\
\text { V/T/O: } 1 / 4 / 0 \\
\text { Mean age: } \\
56 \text { yrs }\end{array}$ & $\begin{array}{l}\text { Prospective } \\
\text { RCT cross- } \\
\text { over design. } \\
\text { Assessed at } 3 \\
\text { weeks } \\
\text { acclimatisation. }\end{array}$ & $\begin{array}{l}\text { TSB: GI: VS } \\
\text { SSB: GI: PS }\end{array}$ & $\begin{array}{l}\text { Function: } \\
\text { Step-count } \\
\underline{\text { QoL: }} \\
\text { PEQ } 3 \text { subscales }\end{array}$ & $\begin{array}{l}\text { SSB significantly } \\
\text { higher usage } \\
(p=0.0056)\end{array}$ \\
\hline $\begin{array}{l}\text { Manucharian } \\
\text { SR. 201153 } \\
\text { Experimental }\end{array}$ & $\begin{array}{l}\text { Prosthetic } \\
\text { facility in } \\
\text { USA }\end{array}$ & $\begin{array}{l}n=36 \\
\text { Aetiology: } \\
\text { V/T/O: } \\
30 / 6 / 0 \\
\text { Mean age: } \\
\text { unknown }\end{array}$ & $\begin{array}{l}\text { Retrospective } \\
\text { Non-RCT } \\
\text { Double post- } \\
\text { test, 2-arm } \\
\text { design. } \\
\text { Assessed after } \\
\text { fitting, \& 1- } \\
\text { month } \\
\text { acclimatisation. }\end{array}$ & $\begin{array}{l}\text { TSB: Fl: SI- } \\
\text { vS } \\
n=15 \\
\text { SSB: Fl: SI- } \\
\text { vS } \\
n=21\end{array}$ & $\frac{\text { Comfort \& pain: }}{\text { SCS }}$ & $\begin{array}{l}\text { Results indicated } \\
\text { comfort in SSB } \\
\text { group higher than } \\
\text { TSB group. }\end{array}$ \\
\hline $\begin{array}{l}\text { Engsberg et al } \\
2006^{50} \\
\text { Experimental }\end{array}$ & $\begin{array}{l}\text { Outpatient } \\
\text { setting in } \\
\text { USA }\end{array}$ & $\begin{array}{l}\mathrm{n}=43 \\
\text { Aetiology: } \\
\text { V/T/O: } \\
\text { unknown } \\
\text { Mean age: } \\
47 \text { years } \\
>2 \text { years } \\
\text { prosthetic } \\
\text { use. }\end{array}$ & $\begin{array}{l}\text { RCT Cross- } \\
\text { over design. } \\
\text { Assessed at } 4 \\
\text { weeks } \\
\text { acclimatisation. }\end{array}$ & $\begin{array}{l}\text { TSB } \\
\text { SSB }\end{array}$ & $\begin{array}{l}\text { Function: } \\
\text { Walk speed } \\
\text { Stride length } \\
\text { Cadence } \\
\text { Gait-asymmetries } \\
\text { QoL: } \\
\text { PEQ } 9 \text { subscales }\end{array}$ & $\begin{array}{l}\text { No difference in gait } \\
\text { parameters } \\
\text { No difference } \\
\text { No difference } \\
\text { No difference } \\
\text { No difference } \\
\text { No difference in } \\
\text { QoL domain } \\
\text { No diff except } \\
\text { perceived response } \\
\text { (p<0.00) in favour } \\
\text { SSB. }\end{array}$ \\
\hline
\end{tabular}




\begin{tabular}{|c|c|c|c|c|c|c|}
\hline & & & & & $\begin{array}{l}\text { Preference } \\
\text { Energy } \\
\text { expenditure: } \\
\mathrm{O}_{2} \text { uptake }\end{array}$ & $\begin{array}{l}\text { TSB: } 25 / 43, \text { SSB: } \\
16 / 43 \\
\text { No difference in } \\
\text { energy expenditure }\end{array}$ \\
\hline $\begin{array}{l}\text { Datta et al } \\
1996^{56} \\
\text { Descriptive } \\
\text { Case series }\end{array}$ & $\begin{array}{l}\text { Outpatient } \\
\text { hospital } \\
\text { setting in } \\
\text { England. }\end{array}$ & $\begin{array}{l}n=54 \\
\text { Aetiology: } \\
V / T / O: \\
11 / 27 / 16 \\
\text { Mean age: } \\
48 \text { years }\end{array}$ & $\begin{array}{l}\text { Retrospective } \\
\text { convenience } \\
\text { sample. } \\
\text { Follow-up 2- } \\
104 \text { weeks. }\end{array}$ & $\begin{array}{l}\text { TSB: GI: PS } \\
\text { SSB: FI: SC- } \\
\text { S }\end{array}$ & $\begin{array}{l}\text { Comfort \& pain: } \\
\\
\text { Wear-comfort: } \\
\text { author-derived } \\
\text { survey }\end{array}$ & $\begin{array}{l}\text { No significant } \\
\text { difference }(p=0.679) \\
\text { between socket } \\
\text { designs } \\
\text { TSB: } 53 \text { responses, } \\
\text { mean score } 3.50 \text {. } \\
\text { SSB: } 51 \text { responses, } \\
\text { mean score } 2.82 \text {. }\end{array}$ \\
\hline $\begin{array}{l}\text { Yigiter et al } \\
2002^{55} \\
\text { Q-Experimental }\end{array}$ & $\begin{array}{l}\text { Inpatient } \\
\text { rehabilitation } \\
\text { setting, in } \\
\text { Turkey. }\end{array}$ & $\begin{array}{l}n=20 \\
\text { Aetiology: } \\
V / T / O: \\
0 / 20 / 0 \\
\text { Mean age: } \\
27 \text { years }\end{array}$ & $\begin{array}{l}\text { Before and } \\
\text { after study. } \\
\text { Assessed after } \\
10 \text { days gait } \\
\text { training and } \\
\text { acclimatisation. }\end{array}$ & $\begin{array}{l}\text { TSB: GI: PS } \\
\text { SSB: FI: SC- } \\
\text { S }\end{array}$ & $\begin{array}{l}\text { Function: } \\
\text { Walk speed } \\
\text { Stride length } \\
\text { Step width } \\
\text { Cadence } \\
\text { Gait-asymmetries } \\
\text { Balance } \\
\text { Weight bearing } \\
\text { ADL analysis } \\
\text { QoL: } \\
\text { Preference } \\
\text { Other: } \\
\text { Prosthetic Mass }\end{array}$ & $\begin{array}{l}\text { TSB effective in gait } \\
\text { parameters } \\
\text { Faster with TSB } \\
(\mathrm{p}<0.05) \\
\text { Increased with TSB, } \\
\text { not significant } \\
\text { TSB narrower } \\
\text { ( } \mathrm{p}<0.05) \\
\text { TSB increased } \\
\text { steps/min ( }<<0.05) \\
\text { TSB more } \\
\text { symmetrical } \\
\text { TSB improved } \\
\text { balance ( } p<0.05) \\
\text { TSB closer to } \\
\text { normal ( } p<0.05) \\
7 / 9 \text { tasks improved } \\
\text { with TSB ( } p<0.05) \\
\text { TSB favoured in } \\
\text { QoL domain } \\
75 \% \text { of subjects } \\
\text { chose to retain TSB } \\
\text { TSB significantly } \\
\text { lighter ( } p<0.05)\end{array}$ \\
\hline $\begin{array}{l}\text { Hachisuka et al } \\
1998^{48 A} \\
\text { Q-Experimental }\end{array}$ & $\begin{array}{l}\text { Outpatient } \\
\text { setting, } \\
\text { Japan } \\
\text { hospital. }\end{array}$ & $\begin{array}{l}n=23 \\
\text { Aetiology: } \\
\text { V/T/O: } \\
\text { unknown } \\
\text { Mean age: } \\
44 \text { years }\end{array}$ & $\begin{array}{l}\text { Before and } \\
\text { after study. }\end{array}$ & $\begin{array}{l}\text { TSB: GI: PS } \\
\text { SSB: FI: SC- } \\
\text { S }\end{array}$ & $\begin{array}{l}\text { QoL: } \\
\text { Preference }\end{array}$ & $\begin{array}{l}\text { TSB favoured in } \\
\text { QoL domain } \\
\text { TSB: } 15 / 23, \text { SSB: } \\
8 / 23\end{array}$ \\
\hline $\begin{array}{l}\text { Hachisuka et al } \\
1998^{48 B} \\
\text { Q-Experimental }\end{array}$ & $\begin{array}{l}\text { Inpatient } \\
\text { setting, } \\
\text { Japan } \\
\text { hospital. }\end{array}$ & $\begin{array}{l}n=9 \\
\text { Aetiology: } \\
V / T / O: \\
\text { unknown } \\
\text { Mean age: } \\
44 \text { years. }\end{array}$ & $\begin{array}{l}\text { Before and } \\
\text { after design. } \\
\text { Subjects used } \\
\text { SSB \& TSB } \\
\text { alternating 3-4 } \\
\text { days. } \\
\text { Assessed at } 2 \\
\text { months use. }\end{array}$ & $\begin{array}{l}\text { TSB: GI: PS } \\
\text { SSB: FI: SC- } \\
\text { S }\end{array}$ & $\begin{array}{l}\text { QoL: } \\
\text { Preference }\end{array}$ & $\begin{array}{l}\text { TSB favoured in } \\
\text { QoL domain } \\
\text { TSB: 9/9, SSB: 0/9 }\end{array}$ \\
\hline
\end{tabular}




\begin{tabular}{|c|c|c|c|c|c|c|}
\hline $\begin{array}{l}\text { Astrom I. and } \\
\text { Stenstrom A. } \\
2004^{54} \\
\text { Q-Experimental }\end{array}$ & $\begin{array}{l}\text { Outpatient } \\
\text { setting in } \\
\text { Sweden. }\end{array}$ & $\begin{array}{l}\mathrm{n}=7 \\
\text { Aetiology: } \\
\text { V/T/O: 3/0/4 } \\
\text { Mean age: } \\
46 \text { yrs } \\
>\text { 1year } \\
\text { rehabilitation } \\
\text { Activity K2+ }\end{array}$ & $\begin{array}{l}\text { Before and } \\
\text { after design. } \\
\text { Assessed at } \\
\text { baseline \& } 2 \\
\text { months } \\
\text { acclimatisation. }\end{array}$ & $\begin{array}{l}\text { TSB: GI: SI- } \\
\text { vS } \\
\text { SSB: FI/GI: } \\
\text { SC-S }\end{array}$ & $\begin{array}{l}\frac{\text { Function: }}{\text { Walk speed }} \\
\underline{\text { QoL: }} \\
\text { Preference }\end{array}$ & $\begin{array}{l}\text { Increased } \\
\text { meters/sec with } \\
\text { TSB } \\
\text { TSB favoured in } \\
\text { QoL domain } \\
\text { TSB: } 7 / 7, \text { SSB: 0/7 }\end{array}$ \\
\hline
\end{tabular}

Abbreviation: Q: Quasi, TSB: total surface bearing; SSB: specific surface bearing; GI: Gel Interface; FI: Foam Interface; PS: Pin Suspension; SC-S: Supracondular suspension; SI-vS: sleeve and valve suspension; VS: vacuum suspension, Aetiology=V: vascular, T: traumatic, O: other, RCT: Randomised Controlled Trial, QoL: Quality of Life, PEQ: prosthesis Evaluation Questionnaire, ADL: Activities of daily living, SCS: Socket Comfort Score, BPI: Brief Pain Inventory; Yrs: years 


\section{Appendix VIII: List of study findings}

\begin{tabular}{|c|c|c|c|c|c|}
\hline \multirow[t]{2}{*}{ Health } & \multirow[t]{2}{*}{ Outcome measured } & \multirow[t]{2}{*}{ Citation } & \multicolumn{3}{|l|}{ Findings } \\
\hline & & & Analysis 1 & Analysis 2 & Analysis 3 \\
\hline \multirow[t]{12}{*}{ Function } & Walk speed & $24,50,52,54,55$ & No difference & TSB\# & TSB\# \\
\hline & Stride length & $23,24,50,52,55$ & No difference & & \\
\hline & Step width & 55 & TSB\# & & \\
\hline & Temporal asymmetry & $24,52,55$ & No difference & & \\
\hline & Spatial asymmetry & $24,50,52$ & No difference & & \\
\hline & Cadence & $24,50,55$ & No difference & TSB\# & \\
\hline & Balance & 55 & TSB\# & & \\
\hline & Weight bearing & 55 & TSB\# & & \\
\hline & Activities of daily living & 52,55 & TSB\# & & \\
\hline & Step Count & 23,51 & SSB \# & & \\
\hline & Wear time & 23 & SSB \# & & \\
\hline & Ambulation & $23,50,51$ & No difference & & \\
\hline \multirow[t]{6}{*}{ Pain/comfort } & Socket Comfort Score & $23,24,53$ & No difference & SSB & \\
\hline & Residual Limb Health & $23,50,51$ & No difference & & \\
\hline & Brief Pain Inventory & 23 & No difference & & \\
\hline & Wear comfort & 56 & No difference & & \\
\hline & Pain & 52 & No difference & & \\
\hline & Phantom Pain & 52 & No difference & & \\
\hline \multirow[t]{9}{*}{ Quality of life } & Socket preference & $23,48 a, 48 b, 50,52,54,55$ & No difference & TSB & TSB\# \\
\hline & Frustration & $23,50,51$ & No difference & TSB & \\
\hline & Utility & 23,50 & No difference & SSB & \\
\hline & Well-being & 23,50 & No difference & & \\
\hline & Sounds & 23,50 & No difference & & \\
\hline & Social Burden & 23,50 & No difference & & \\
\hline & Perceived Response & 23,50 & SSB\# & & \\
\hline & Appearance & 23,50 & No difference & & \\
\hline & Satisfaction & 52 & No difference & & \\
\hline Energy usage & Oxygen Uptake & 50 & No difference & & \\
\hline \multirow[t]{3}{*}{ Others } & Mass & 52,55 & No difference & TSB\# & \\
\hline & Cost of materials & 24,52 & TSB\# & & \\
\hline & Time to manufacture & 24,52 & SSB\# & & \\
\hline
\end{tabular}

Abbreviation: TSB: total surface bearing; SSB: specific surface bearing; \#: statistical significance 


\section{Appendix IX: JBI Levels of evidence}

\begin{tabular}{|c|c|c|c|c|c|}
\hline \begin{tabular}{|l|} 
Levels of \\
Evidence
\end{tabular} & $\begin{array}{c}\text { Feasibility } \\
\mathrm{F}(1-4)\end{array}$ & \begin{tabular}{|c|} 
Appropriateness \\
A(1-4)
\end{tabular} & $\begin{array}{c}\text { Meaningfulness } \\
M(1-4)\end{array}$ & Effectiveness E(1-4) & Economic Evidence \\
\hline 1 & \begin{tabular}{|l|} 
Metasynthesis \\
of research \\
with \\
unequivocal \\
synthesised \\
findings
\end{tabular} & $\begin{array}{l}\text { Metasynthesis of } \\
\text { research with } \\
\text { unequivocal } \\
\text { synthesised } \\
\text { findings }\end{array}$ & \begin{tabular}{|l|} 
Metasynthesis \\
of research with \\
unequivocal \\
synthesised \\
findings
\end{tabular} & \begin{tabular}{|l|} 
Meta-analysis(with \\
homogeneity) of \\
experimental studies (eg \\
RCT with concealed \\
randomisation) OR One or \\
more large experimental \\
studies with narrow \\
confidence intervals
\end{tabular} & $\begin{array}{l}\text { Metasynthesis (with homogeneity) } \\
\text { of evaluations of important } \\
\text { alternative interventions } \\
\text { comparing all clinically relevant } \\
\text { outcomes against appropriate cost } \\
\text { measurement, and including a } \\
\text { clinically sensible sensitivity } \\
\text { analysis }\end{array}$ \\
\hline 2 & \begin{tabular}{|l} 
Metasynthesis \\
of research \\
with credible \\
synthesised \\
findings
\end{tabular} & $\begin{array}{l}\text { Metasynthesis of } \\
\text { research with } \\
\text { credible } \\
\text { synthesised } \\
\text { findings }\end{array}$ & \begin{tabular}{|l|} 
Metasynthesis \\
of research with \\
credible \\
synthesised \\
findings
\end{tabular} & \begin{tabular}{|l|} 
One or more smaller RCTs \\
with wider confidence \\
intervals OR Quasi- \\
experimental \\
studies(without \\
randomisation)
\end{tabular} & $\begin{array}{l}\text { Evaluations of important } \\
\text { alternative interventions } \\
\text { comparing all clinically relevant } \\
\text { outcomes against appropriate cost } \\
\text { measurement, and including a } \\
\text { clinically sensible sensitivity } \\
\text { analysis }\end{array}$ \\
\hline 3 & \begin{tabular}{|l} 
a. \\
Metasynthesis \\
of \\
text/opinion \\
with credible \\
synthesised \\
findings \\
b. One or \\
more single \\
research \\
studies of \\
high quality
\end{tabular} & \begin{tabular}{|l|} 
a. Metasynthesis \\
of text/opinion \\
with credible \\
synthesised \\
findings \\
b. One or more \\
single research \\
studies of high \\
quality
\end{tabular} & \begin{tabular}{|l|} 
a. Metasynthesis \\
of text/opinion \\
with credible \\
synthesised \\
findings \\
b. One or more \\
single research \\
studies of high \\
quality
\end{tabular} & $\begin{array}{l}\text { a. Cohort studies (with } \\
\text { control group) } \\
\text { b. Case-controled } \\
\text { c. Observational } \\
\text { studies(without control } \\
\text { group) }\end{array}$ & $\begin{array}{l}\text { Evaluations of important } \\
\text { alternative interventions } \\
\text { comparing a limited number of } \\
\text { appropriate cost measurement, } \\
\text { without a clinically sensible } \\
\text { sensitivity analysis }\end{array}$ \\
\hline 4 & $\begin{array}{l}\text { Expert } \\
\text { opinion }\end{array}$ & Expert opinion & Expert opinion & $\begin{array}{l}\text { Expert opinion, or } \\
\text { physiology bench research, } \\
\text { or consensus }\end{array}$ & $\begin{array}{l}\text { Expert opinion, or based on } \\
\text { economic theory }\end{array}$ \\
\hline
\end{tabular}

